\title{
2013 ESC Guidelines on cardiac pacing and cardiac resynchronization therapy
}

\section{The Task Force on cardiac pacing and resynchronization therapy of the European Society of Cardiology (ESC). Developed in collaboration with the European Heart Rhythm Association (EHRA).}

Authors/Task Force Members: Michele Brignole (Chairperson) (Italy)*, Angelo Auricchio (Switzerland), Gonzalo Baron-Esquivias (Spain), Pierre Bordachar (France), Giuseppe Boriani (Italy), Ole-A Breithardt (Germany), John Cleland (UK), Jean-Claude Deharo (France), Victoria Delgado (Netherlands), Perry M. Elliott (UK), Bulent Gorenek (Turkey), Carsten W. Israel (Germany), Christophe Leclercq (France), Cecilia Linde (Sweden), Lluís Mont (Spain), Luigi Padeletti (Italy), Richard Sutton (UK), Panos E. Vardas (Greece)

ESC Committee for Practice Guidelines (CPG): Jose Luis Zamorano (Chairperson) (Spain), Stephan Achenbach (Germany), Helmut Baumgartner (Germany), Jeroen J. Bax (Netherlands), Héctor Bueno (Spain), Veronica Dean (France), Christi Deaton (UK), Cetin Erol (Turkey), Robert Fagard (Belgium), Roberto Ferrari (Italy), David Hasdai (Israel), Arno W. Hoes (Netherlands), Paulus Kirchhof (Germany/UK), Juhani Knuuti (Finland), Philippe Kolh (Belgium), Patrizio Lancellotti (Belgium), Ales Linhart (Czech Republic), Petros Nihoyannopoulos (UK), Massimo F. Piepoli (Italy), Piotr Ponikowski (Poland), Per Anton Sirnes (Norway), Juan Luis Tamargo (Spain), Michal Tendera (Poland), Adam Torbicki (Poland), William Wijns (Belgium), Stephan Windecker (Switzerland).

Document Reviewers: Paulus Kirchhof (CPG Review Coordinator) (Germany/UK), Carina Blomstrom-Lundqvist (CPG Review Coordinator) (Sweden), Luigi P. Badano (Italy), Farid Aliyev (Azerbaijan), Dietmar Bänsch (Germany), Helmut Baumgartner (Germany), Walid Bsata (Syria), Peter Buser (Switzerland), Philippe Charron (France), Jean-Claude Daubert (France), Dan Dobreanu (Romania), Svein Faerestrand (Norway), David Hasdai (Israel), Arno W. Hoes (Netherlands), Jean-Yves Le Heuzey (France), Hercules Mavrakis (Greece), Theresa McDonagh (UK), Jose Luis Merino (Spain), Mostapha M. Nawar (Egypt), Jens Cosedis Nielsen (Denmark), Burkert Pieske (Austria), Lidija Poposka (The Former Yugoslav Republic of Macedonia), Frank Ruschitzka (Switzerland), Michal Tendera (Poland), Isabelle C. Van Gelder (Netherlands), Carol M. Wilson (Ireland).

The disclosure forms of the authors and reviewers are available on the ESC website www.escardio.org/guidelines

\footnotetext{
* Corresponding author. Michele Brignole, Department of Cardiology, Ospedali del Tigullio, Via Don Bobbio 25, IT-16033 Lavagna, (GE) Italy. Tel: + 390185329569 , Fax: + 390185306 506, Email: mbrignole@ASL4.liguria.it

tOther ESC entities having participated in the development of this document:

Associations: Acute Cardiovascular Care Association (ACCA), Heart Failure Association (HFA), European Association of Cardiovascular Imaging (EACVI)

Working Groups: Myocardial and Pericardial Diseases

Council: Cardiology Practice

The content of these European Society of Cardiology (ESC) Guidelines has been published for personal and educational use only. No commercial use is authorized. No part of the ESC Guidelines may be translated or reproduced in any form without written permission from the ESC. Permission can be obtained upon submission of a written request to Oxford University Press, the publisher of the European Heart Journal and the party authorized to handle such permissions on behalf of the ESC.

Disclaimer. The ESC Guidelines represent the views of the ESC and were arrived at after careful consideration of the available evidence at the time they were written. Health professionals are encouraged to take them fully into account when exercising their clinical judgement. The guidelines do not, however, override the individual responsibility of health professionals to make appropriate decisions in the circumstances of the individual patients, in consultation with that patient and, where appropriate and necessary, the patient's guardian or carer. It is also the health professional's responsibility to verify the rules and regulations applicable to drugs and devices at the time of prescription.

(C) The European Society of Cardiology 2013. All rights reserved. For permissions please email: journals.permissions@oup.com
} 


\section{Table of Contents}

Abbreviations and acronyms ................. 2

Abbreviations: ...................... 2

Acronyms of the trials referenced in the recommendations or reported in the tables: . . . . . . . . . . . . . . . . 3

1. Preamble ........................ 4

2. Indications for pacing .................. 5

2.1 Epidemiology, natural history, pathophysiology,

classification, and diagnosis of bradyarrhythmias considered for permanent cardiac pacing therapy ... . . . . . . . . . . 5

2.2 Persistent bradycardia . . . . . . . . . . . . . . 7

2.2.1 Indications for pacing . . . . . . . . . . . . 7

2.2 .2 Choice of pacing mode. . . . . . . . . . . . . . . 9 9

2.3 Intermittent (documented) bradycardia . . . . . . . . . . . 11

2.3.1 Indications for pacing . . . . . . . . . . . . . . 11

2.3 .2 Choice of pacing mode. . . . . . . . . . . . . . . . 13

Section 2.4 Suspected (undocumented) bradycardia . . . . . . 14

2.4.1 Bundle branch block . . . . . . . . . . . . . . . . . . . 14

2.4 .2 Reflex syncope. . . . . . . . . . . . . . . . . . 16

2.4.3 Unexplained syncope (and fall) . . . . . . . . . . . 18

3. Indications for cardiac resynchronization therapy . . . . . . . . . 19

3.1 Epidemiology, prognosis, and pathophysiology of heart failure suitable for cardiac resynchronization therapy . . . . . . 19

Section 3.2 Patients in sinus rhythm . . . . . . . . . . . . . . . 20 3.2.1 Indications for cardiac resynchronization therapy . . . 20

3.2.1.1 Patients in New York Heart Association functional class III-IV . . . . . . . . . . . . . . . . . 20

3.2.1.2 Patients in New York Heart Association

functional class $\mid-\| \ldots \ldots 20$

3.2.1.3 Patient selection: role of imaging techniques to evaluate mechanical dyssynchrony criteria to select patients for cardiac resynchronization therapy . . . . . . . 23

3.2.2 Choice of pacing mode (and cardiac resynchronization therapy optimization) . . . . . . . . . . . . . . . 23

Section 3.3 Patients in atrial fibrillation . . . . . . . . . . . . 26

3.3.1 Patients with heart failure, wide QRS, and reduced ejection fraction . . . . . . . . . . . . . . 26

3.3.2 Patients with uncontrolled heart rate who are candidates for atrioventricular junction ablation . . . . . . . . 27

3.4 Patients with heart failure and conventional pacemaker

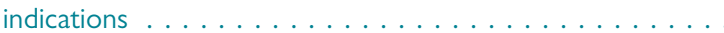

3.4.1 Patients with an indication for upgrading from conventional pacemaker or implantable cardioverter defibrillator to cardiac resynchronization therapy devices 3.4.2 De novo cardiac resynchronization therapy pacing in patients with conventional indication for anti-bradycardia pacing. 26
Section 3.5 Back-up implantable cardioverter defibrillator in patients indicated for cardiac resynchronization therapy . . . . 31

3.5.1 Benefit of adding cardiac resynchronization therapy in patients with indications for implantable cardioverter defibrillator ..................... 31

3.5.2 Benefit of adding implantable cardioverter defibrillator in patients with indications for cardiac resynchronization therapy. . . . . . . . . . . . . . . . . . 31

4. Indications for pacing in specific conditions . . . . . . . . . . 33

4.1 Pacing in acute myocardial infarction . . . . . . . . . . 33

4.2 Pacing after cardiac surgery, transcatheter aortic valve implantation, and heart transplantation . . . . . . . . . . . 34

4.3 Pacing and cardiac resynchronization therapy in children and in congenital heart disease . . . . . . . . . . . 35

4.4 Pacing in hypertrophic cardiomyopathy . . . . . . . . . . . 36

4.5 Pacing in rare diseases . . . . . . . . . . . . . . 37

4.5 .1 Long QT syndrome . . . . . . . . . . . . . . . . . . . 38

4.5 .2 Muscular dystrophies . . . . . . . . . . . . . . . . 38

4.5 .3 Mitochondrial cytopathies . . . . . . . . . . . . . . 38

4.5 .4 Metabolic disorders . . . . . . . . . . . . . . . 39

4.6 Pacing in pregnancy . . . . . . . . . . . . . . . . . . . 39

4.7 Pacing for first-degree atrioventricular block (haemodynamic) ...................... 39

4.8 Algorithms for prevention and termination of atrial

arrhythmias by pacing . . . . . . . . . . . . . . . . . 39

5. Complications of pacing and CRT implantation . . . . . . . . . . . 41

6. Management considerations . . . . . . . . . . . . . . . . . . . 41

6.1 Pacing from alternative right ventricular sites . . . . . . . . . 41

6.2 Re-implantation of pacemaker/cardiac resynchronization therapy after device explantation for infection . . . . . . . . . 42

6.3 Magnetic resonance imaging in patients with implanted

cardiac devices . . . . . . . . . . . . . . . . . . . . . 42

6.4 Emergency (transvenous) temporary pacing . . . . . . . . . 44

6.5 Remote management of arrhythmias and device . . . . . . 44

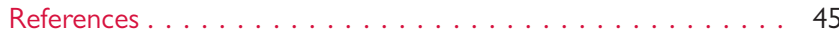

\section{Abbreviations and acronyms}

\section{Abbreviations}

1st AV First-degree atrioventricular block

AF atrial fibrillation

AT atrial tachyarrhythmia

ATP Anti-tachycardia pacing

\section{1}

1

(

3

3

4

(3)

36

36
8

8

9
39

9

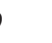

1

1

1

2

4

4




$\begin{array}{ll}\text { AV } & \text { atrioventricular } \\ \text { BBB } & \text { bundle branch block } \\ \text { CHF } & \text { congestive heart failure } \\ \text { CI } & \text { confidence interval } \\ \text { CPG } & \text { Committee for Practice Guidelines } \\ \text { CRT } & \text { cardiac resynchronization therapy } \\ \text { CRT-D } & \text { cardiac resynchronization therapy and defibrillator } \\ \text { CRT-P } & \text { cardiac resynchronization therapy and pacemaker } \\ \text { ECG } & \text { electrocardiogram } \\ \text { EDMD } & \text { Emery-Dreifuss muscular dystrophy } \\ \text { EF } & \text { ejection fraction } \\ \text { EPS } & \text { electrophysiological study } \\ \text { ESC } & \text { European Society of Cardiology } \\ \text { HCM } & \text { hypertrophic cardiomyopathy } \\ \text { HF } & \text { heart failure } \\ \text { HR } & \text { hazard ratio } \\ \text { HV } & \text { His-ventricular } \\ \text { ICD } & \text { implantable cardioverter defibrillator } \\ \text { ILR } & \text { implantable loop recorder } \\ \text { IVCD } & \text { intraventricular conduction delay } \\ \text { LBBB } & \text { left bundle branch block } \\ \text { LQTS } & \text { long QT syndrome } \\ \text { LV } & \text { left ventricular } \\ \text { LVEF } & \text { left ventricular ejection fraction } \\ \text { LVSD } & \text { left ventricular systolic dysfunction } \\ \text { MR } & \text { mitral regurgitation } \\ \text { MRI } & \text { magnetic resonance imaging } \\ \text { NYHA } & \text { New York Heart Association } \\ \text { PM } & \text { pacemaker } \\ \text { OR } & \text { odds ratio } \\ \text { QALY } & \text { quality-adjusted life year } \\ \text { RBBB } & \text { right bundle branch block } \\ \text { RCT } & \text { randomized controlled trial } \\ \text { RV } & \text { right ventricular } \\ \text { SB } & \text { sinus bradycardia } \\ \text { SNRT } & \text { sinus node recovery time } \\ \text { SR } & \text { sinus rhythm } \\ \text { SSS } & \text { sick sinus syndrome } \\ \text { tAVI } & \text { transcatheter aortic valve implantation } \\ & \text { interventricular (delay) } \\ \text { VF } & \end{array}$

\section{Acronyms of the trials referenced in the recommendations or reported in the tables}

$\begin{array}{ll}\text { ADEPT } & \begin{array}{l}\text { ADvanced Elements of Pacing Randomized } \\ \text { Controlled Trial }\end{array} \\ \text { ADOPT } & \text { Atrial Dynamic Overdrive Pacing Trial } \\ \text { AOPS } & \text { Atrial Overdrive Pacing Study } \\ \text { APAF } & \text { Ablate and Pace in Atrial Fibrillation } \\ \text { ASSERT } & \text { ASymptomatic Atrial Fibrillation and Stroke } \\ & \text { Evaluation in Pacemaker Patients and the } \\ & \text { Atrial Fibrillation Reduction Atrial Pacing Trial } \\ \text { ATTEST } & \text { ATrial Therapy Efficacy and Safety Trial }\end{array}$

\begin{tabular}{|c|c|}
\hline AVAIL CLS/CRT & $\begin{array}{l}\text { AV Node Ablation with CLS and CRT Pacing } \\
\text { Therapies for Treatment of AF trial }\end{array}$ \\
\hline B4 & Bradycardia detection in Bundle Branch Block \\
\hline BELIEVE & $\begin{array}{l}\text { Bi vs. Left Ventricular Pacing: an International } \\
\text { Pilot Evaluation on Heart Failure Patients with } \\
\text { Ventricular Arrhythmias }\end{array}$ \\
\hline BIOPACE & $\begin{array}{l}\text { Biventricular pacing for atrioventricular block } \\
\text { to prevent cardiac desynchronization }\end{array}$ \\
\hline BLOCK-HF & $\begin{array}{l}\text { Biventricular versus right ventricular pacing in } \\
\text { patients with } \mathrm{AV} \text { block }\end{array}$ \\
\hline B-LEFT & $\begin{array}{l}\text { Biventricular versus LEFT Univentricular Pacing } \\
\text { with ICD Back-up in Heart Failure Patients }\end{array}$ \\
\hline CARE-HF & CArdiac REsynchronization in Heart Failure \\
\hline CLEAR & $\begin{array}{l}\text { CLinical Evaluation on Advanced Resynchroni- } \\
\text { zation }\end{array}$ \\
\hline COMBAT & $\begin{array}{l}\text { COnventional vs. Biventricular Pacing in Heart } \\
\text { Failure and Bradyarrhythmia }\end{array}$ \\
\hline COMPANION & $\begin{array}{l}\text { COmparison of Medical Therapy, Pacing and } \\
\text { Defibrillation in Heart Failure }\end{array}$ \\
\hline
\end{tabular}

DANPACE

DECREASE-HF

DANish Multicenter Randomized Trial on Single Lead Atrial PACing vs. Dual Chamber Pacing in Sick Sinus Syndrome

The Device Evaluation of CONTAK RENEWAL 2 and EASYTRAK 2: Assessment of Safety and Effectiveness in Heart Failure

FREEDOM Optimization Study Using the QuickOpt Method

GREATER-EARTH Evaluation of Resynchronization Therapy for Heart Failure in Patients with a QRS Duration GREATER Than $120 \mathrm{~ms}$

LESSER-EARTH Evaluation of Resynchronization Therapy for Heart Failure in Patients with a QRS Duration Lower Than $120 \mathrm{~ms}$

HOBIPACE HOmburg Blventricular PACing Evaluation

IN-CHF

ISSUE Italian Network on Congestive Heart Failure International Study on Syncope of Unexplained Etiology

MADIT

MIRACLE

Multicenter Automatic Defibrillator Trial

Multicenter InSync RAndomized CLinical Evaluation

MOST

MUSTIC

OPSITE

PACE

PAVE

PATH-CHF

PIPAF

PIRAT

POT

PREVENT-HF PREventing VENTricular Dysfunction in PacePROSPECT MOde Selection Trial in Sinus-Node Dysfunction

MUltisite STimulation In Cardiomyopathies

Optimal Pacing SITE

Pacing to Avoid Cardiac Enlargement

Left Ventricular-Based Cardiac Stimulation Post AV Nodal Ablation Evaluation

PAcing THerapies in Congestive Heart Failure II Study Group

Pacing In Prevention of Atrial Fibrillation Study Prevention of Immediate Reinitiation of Atrial Tachyarrhythmias

Prevention Or Termination Study maker Patients Without Advanced Heart Failure PRedictors OfResponse to Cardiac Resynchronization Therapy 
RAFT

Resynchronization-Defibrillation for Ambulatory Heart Failure Trial

RethinQ

REVERSE

SAFARI

SCD HeFT

SMART-AV

SYDIT

SYNPACE

TARGET

THEOPACE

VASIS-PM

V-HeFT

VPSII

\section{Addenda}

Additional references are mentioned with ' $w$ ' in the main text and can be found on the online addenda along with 5 figures $(1,6,7,9,11,12)$ and 10 tables $(3,4,5,9,11,12,19,21,22,23)$. They are available on the ESC website only at http://www.escardio.org/guidelines-surveys/ esc-guidelines/Pages/cardiac-pacing-and-cardiac-resynchronisationtherapy.aspx

\section{Preamble}

Guidelines summarize and evaluate all available evidence, at the time of the writing process, on a particular issue, with the aim of assisting physicians in selecting the best management strategies for an individual patient with a given condition, taking into account the impact on outcome, as well as the risk-benefit ratio of particular diagnostic or therapeutic means. Guidelines are not substitutes, but are complements for textbooks and cover the ESC Core Curriculum topics. Guidelines and recommendations should help physicians to make decisions in their daily practice. However, the final decisions concerning an individual patient must be made by the responsible physician(s).

A great number of guidelines have been issued in recent years by the European Society of Cardiology (ESC), as well as by other societies and organisations. Because of the impact on clinical practice, quality criteria for the development of guidelines have been established, in order to make all decisions transparent to the user. The recommendations for formulating and issuing ESC Guidelines can be found on the ESC website (http://www.escardio.org/ guidelines-surveys/esc-guidelines/about/Pages/rules-writing. aspx). ESC Guidelines represent the official position of the ESC on a given topic and are regularly updated.

Members of this Task Force were selected by the ESC to represent professionals involved with the medical care of patients with this pathology. Selected experts in the field undertook a comprehensive review of the published evidence for diagnosis, management and/or prevention of a given condition, according to ESC Committee for Practice Guidelines (CPG) policy. A critical evaluation of diagnostic and therapeutic procedures was performed, including assessment of the risk-benefit ratio. Estimates of expected health outcomes for larger populations were included, where data exist. The level of evidence and the strength of recommendation of particular treatment options were weighed and graded according to predefined scales, as outlined in Tables 1 and 2.

The experts of the writing and reviewing panels completed Declaration of Interest forms where real or potential sources of conflicts of interest might be perceived. These forms were compiled into one file and can be found on the ESC website (http://www.escardio.org/ guidelines). Any changes in declarations of interest that arise during the writing period must be notified to the ESC and updated. The Task Force received its entire financial support from the ESC without any involvement from healthcare industry.

\section{Table I Classes of recommendations}

\begin{tabular}{|c|c|c|}
\hline $\begin{array}{l}\text { Classes of } \\
\text { recommendations }\end{array}$ & Definition & Suggested wording to use \\
\hline Class I & $\begin{array}{l}\text { Evidence and/or general agreement } \\
\text { that a given treatment or procedure } \\
\text { is beneficial, useful, effective. }\end{array}$ & $\begin{array}{l}\text { Is recommended/is } \\
\text { indicated }\end{array}$ \\
\hline Class II & $\begin{array}{l}\text { Conflicting evidence and/or a } \\
\text { divergence of opinion about the } \\
\text { usefulness/efficacy of the given } \\
\text { treatment or procedure. }\end{array}$ & \\
\hline Class IIa & $\begin{array}{l}\text { Weight of evidence/opinion is in } \\
\text { favour of usefulness/efficacy. }\end{array}$ & Should be considered \\
\hline Class IIb & $\begin{array}{l}\text { Usefulness/efficacy is less well } \\
\text { established by evidence/opinion. }\end{array}$ & May be considered \\
\hline Class III & $\begin{array}{l}\text { Evidence or general agreement that } \\
\text { the given treatment or procedure } \\
\text { is not useful/effective, and in some } \\
\text { cases may be harmful. }\end{array}$ & Is not recommended \\
\hline
\end{tabular}


Table 2 Levels of evidence

\begin{tabular}{|l|l|}
\hline $\begin{array}{l}\text { Level of } \\
\text { evidence A }\end{array}$ & $\begin{array}{l}\text { Data derived from multiple randomized } \\
\text { clinical trials or meta-analyses. }\end{array}$ \\
\hline $\begin{array}{l}\text { Level of } \\
\text { evidence B }\end{array}$ & $\begin{array}{l}\text { Data derived from a single randomized } \\
\text { clinical trial or large non-randomized } \\
\text { studies. }\end{array}$ \\
\hline $\begin{array}{l}\text { Level of } \\
\text { evidence C }\end{array}$ & $\begin{array}{l}\text { Consensus of opinion of the experts and/ } \\
\text { or small studies, retrospective studies, } \\
\text { registries. }\end{array}$ \\
\hline
\end{tabular}

The ESC's CPG supervises and coordinates the preparation of new Guidelines produced by Task Forces, expert groups or consensus panels. The Committee is also responsible for the endorsement process of these Guidelines. The ESC Guidelines undergo extensive review by the CPG and external experts. After appropriate revisions, they are approved by all the experts involved in the Task Force. The finalized document is approved by the CPG for publication in the European Heart Journal.

The task of developing the ESC Guidelines covers not only the integration of the most recent research, but also the creation of educational tools and implementation programmes for the recommendations. To implement the guidelines, condensed pocket editions, summary slides, booklets with essential messages, electronic versions for digital applications (smartphones etc.) are produced. These versions are abridged and thus, if needed, one should always refer to the full text version, which is freely available on the ESC website. The National Societies of the ESC are encouraged to endorse, translate and implement the ESC Guidelines. Implementation programmes are needed, because it has been shown that the outcome of disease may be favourably influenced by the thorough application of clinical recommendations.

Surveys and registries are needed to verify that real-life daily practice is in keeping with what is recommended in the Guidelines, thus completing the loop between clinical research, writing of guidelines and implementing them into clinical practice.

The Guidelines do not, however, override the individual responsibility of health professionals to make appropriate decisions in the circumstances of the individual patients, in consultation with that patient and, where appropriate and necessary, the patient's guardian or carer. It is also the health professional's responsibility to verify the rules and regulations applicable to drugs and devices at the time of prescription.

\section{Indications for pacing}

\subsection{Epidemiology, natural history, pathophysiology, classification, and diagnosis of bradyarrhythmias considered for permanent cardiac pacing therapy}

\section{Epidemiology}

The prevalence of bradyarrhythmias requiring permanent cardiac pacing therapy is unknown, but an approximation can be obtained from the analysis of some large databases. A large variability, between European countries, in number of pacemaker (PM) implantations has been described that may reflect differences in demographics and disease prevalence, but could also reflect under-provision in some (Web Figure 1). ${ }^{\mathrm{w} 1, \mathrm{w} 2}$ On the other hand, it is likely that some patients who receive a pacemaker (PM) do not meet current guideline criteria. More clinical details are available from some national registries (Web Table 3). ${ }^{\text {w3-w8 }}$

\section{Natural history and role of pacing}

Inevitably, knowledge of the natural history of severe bradyarrhythmias comes from very old studies performed at the beginning of the PM era. In some situations, efficacy of pacing is therefore inferred, rather than proven by randomized clinical trials.

\section{Atrioventricular block}

Death in patients with untreated atrioventricular (AV) block is due not only to heart failure (HF) secondary to low cardiac output, but also to sudden cardiac death caused by prolonged asystole or bradycardia-triggered ventricular tachyarrhythmia. Although formal randomized controlled trials (RCTs) of pacing in AV block have not been performed, it is clear from several observational studies that pacing prevents recurrence of syncope and improves survival in adults and in children (see section 4.3). ${ }^{\text {w9-w13 }}$

In patients with first-degree AV block and type I second-degree AV block with marked PR prolongation, small uncontrolled trials have suggested symptomatic and functional improvement, with normalization of the PR interval with dual-chamber pacing (AV resynchronization). ${ }^{\text {w14-w16 }}$

\section{Sinus node dysfunction}

There is no evidence that cardiac pacing prolongs survival in patients with sinus node dysfunction. Indeed, total survival and the risk of sudden cardiac death of patients with sick sinus syndrome (SSS) (irrespective of symptoms) are similar to that of the general population. ${ }^{1, w 17, w 18}$ Nevertheless, systemic thromboembolism is common in untreated patients with SSS. In a literature review, ${ }^{\text {w18 }}$ systemic embolism occurred in $15.2 \%$ of unpaced SSS patients, compared with $1.3 \%$ in age-matched controls; the incidence of atrial fibrillation (AF) in unpaced patients was $8.2 \%$ at initial diagnosis and increased to $15.8 \%$ during a mean follow-up of 38 months. There are no controlled trials comparing embolic events in untreated and treated patients. In the same review, ${ }^{\text {w18 }}$ embolism with VVI PM was $12.3 \%$, which was not very different from the incidence of untreated patients. In a systematic review of large randomized trials, ${ }^{2}$ there was a significant reduction in stroke (hazard ratio [HR]: 0.81) and AF (HR: 0.80) with atrial-based pacing (AAI or DDD) compared with $\mathrm{VVI}$ pacing; these effects were more pronounced in patients with sinus node dysfunction than in those without it, but were not associated with a survival benefit. Finally, the recent DANish Multicenter Randomized Trial on Single Lead Atrial PACing vs. Dual Chamber Pacing in Sick Sinus Syndrome (DANPACE) study showed that AAIR pacing is associated with a higher incidence of paroxysmal AF than DDDR pacing. ${ }^{3}$

\section{Extrinsic (functional) bradycardia}

Since the prognosis is benign - similar to that of the general population - the only reason for cardiac pacing is to prevent (traumatic) recurrent syncope. 


\section{Pathophysiology and classification}

Bradyarrhythmias requiring cardiac pacing can be caused by a variety of aetiologies (Web Table 4) and the early identification of a potentially reversible cause is the first step towards treatment. Among 277 patients referred urgently to an emergency department for compromising bradycardia, adverse drug effects were responsible for bradycardia in $21 \%$, acute myocardial infarction in $14 \%$, intoxication in $6 \%$ and electrolyte disorders in $4 \%$ of cases. ${ }^{w 19}$

In general, when a transient or reversible cause is excluded, the indication for cardiac pacing is determined by the severity of bradycardia, rather than its aetiology. The clinical presentation is more useful for selecting patients for permanent cardiac pacing therapy (Figure 2) and will be followed in these Guidelines.

The main physiological effect of bradycardia is to decrease cardiac output. As long as changes in stroke volume compensate for the decrease in heart rate, patients with profound bradycardia can remain completely asymptomatic. First-degree AV block and type I seconddegree AV block with marked PR prolongation ( $>0.3 \mathrm{~s}$ ) can lead to symptoms, because atrial contraction occurs very early in diastole, at the expense of early diastolic filling, and diastolic mitral regurgitation may occur between the end of atrial filling and the onset of ventricular contraction (see section 4.8). ${ }^{w 14-w 16}$
While the permanent forms of bradyarrhythmia are caused by an intrinsic disease of the sinus node or AV conduction system, the aetiology of intermittent bradyarrhythmia can be difficult to determine. Pure intrinsic (electrophysiological) mechanisms include intermittent/paroxysmal AV block initiated by atrial, His or ventricular premature complexes, increased heart rate (tachy-dependent AV block) or decreased heart rate (brady-dependent AV block), ${ }^{\text {w20,w21 }}$ or sino-atrial block following the termination of tachycardia in the brady-tachy syndrome, which unmasks an impairment of the automatic properties of the sino-atrial node. ${ }^{\text {w22 }}$ When these features are absent, disturbances of the autonomic nervous system or neurohumoral mechanisms, e.g. adenosine metabolism, can explain intermittent bradycardia alone or in conjunction with an intrinsic cardiac abnormality of the sinus node or AV conduction. 4 , w23,w24

In conclusion, whilst persistent bradycardia clearly indicates an intrinsic AV block or SSS, the meaning of intermittent bradycardia is less clear, resulting from variable contributions of intrinsic and extrinsic mechanisms. Often the same event (i.e. intermittent bradycardia) may be diagnosed by one physician as a primary cardiac arrhythmia and by another as a cardio-inhibitory reflex. The problem is further complicated by the fact that the diagnosis of intermittent bradycardia is often only presumed but not documented by electrocardiogram

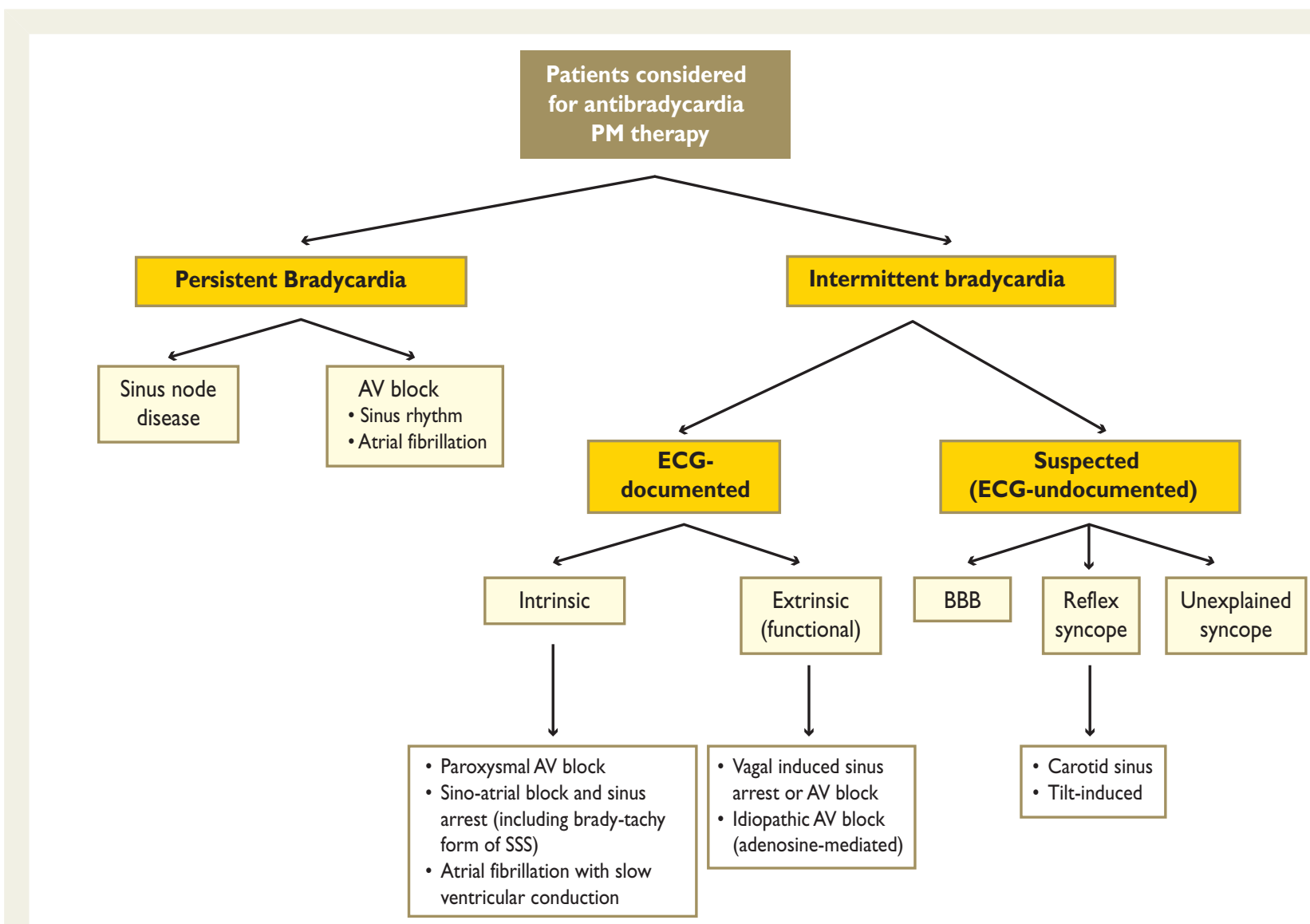

Figure 2 Classification of bradyarrhythmias based on the patient's clinical presentation. AV = atrioventricular; BBB = bundle branch block; $\mathrm{ECG}=$ electrocardiogram; PM = pacemaker; $\mathrm{SSS}=$ sick sinus syndrome. 
(ECG). In general, a reflex mechanism is more likely to be invoked when intermittent bradycardia is not documented, whereas if bradycardia is documented, it will be classified as AV block or SSS.

\section{Diagnosis}

Sinus bradycardia (SB) and AV block can be entirely asymptomatic in young, healthy individuals or during sleep, but patients with sustained or frequent bradyarrhythmia are often symptomatic. Easy fatigability, reduced exercise capacity and symptoms of HF are common in persistent bradyarrhythmia. Subtle symptoms are irritability, lassitude, inability to concentrate, apathy, forgetfulness and dizziness. Dizziness, pre-syncope and syncope are common symptoms with intermittent severe forms of bradyarrhythmias and are due to a sudden decrease in cerebral blood flow (Web Table 5).

The diagnosis of bradyarrhythmia is usually made from a standard ECG when persistent, and from a standard ECG or more prolonged ECG recordings [ambulatory monitoring or implantable loop recorder (ILR)] when intermittent. Provocative testing or an electrophysiological study (EPS) may be required when a bradycardia is suspected but not documented (Figure 2).

Since there is no defined heart rate below which treatment is indicated, correlation between symptoms and bradyarrhythmia is essential when deciding on the need for cardiac pacing therapy. This can be difficult to establish in patients with competing mechanisms for their symptoms - for example, HF or pulmonary disease. Another common dilemma is the patient with persistent bradycardia and intermittent symptoms - for example, syncope in patients with mild persistent SB or permanent AF with low ventricular rate. In selected patients with moderate $\mathrm{SB}$, a prolonged sinus node recovery time (SNRT) at EPS indicates a likely bradyarrhythmic mechanism for syncope. ${ }^{1}$ In general, an attempt to obtain ECG documentation during syncope (symptom-arrhythmia correlation) is warranted (see below).

When an intermittent bradyarrhythmia is suspected but not proven, the suspicion should be corroborated by an ECG documentation of bradyarrhythmia or, alternatively, by laboratory testing. ${ }^{\text {w2 }} 5$ The most useful tests and their diagnostic yield are listed in Table 6.

- ECG monitoring. Short-term monitoring (Holter, telemetry and external loop recorder) is useful, soon after the index episode, in patients who have very frequent symptoms (at least once per week). Since most patients with syncope have infrequent symptoms, recurring over months or years, ILRs are often necessary to establish a diagnosis (Table 7). The diagnostic yield of ILR is a function of the duration of the monitoring. The actuarial diagnostic

Table 6 Diagnosing bradyarrhythmic syncope after the initial evaluation: most useful tests

\begin{tabular}{|l|l|}
\hline $\begin{array}{c}\text { Prolonged electrocardiogram } \\
\text { monitoring strategy }\end{array}$ & \multicolumn{1}{c|}{$\begin{array}{c}\text { Provocative (laboratory) test } \\
\text { strategy }\end{array}$} \\
\hline $\begin{array}{l}\text { - Holter } \\
\text { - External loop recorder } \\
\text { - Remote at-home telemetry } \\
\text { - Implantable loop recorder }\end{array}$ & $\begin{array}{l}\text { - Carotid sinus massage } \\
\text { - Tilt table test }\end{array}$ \\
\hline
\end{tabular}

$\mathrm{ECG}=$ electrocardiogram
Table 7 Suggested ECG monitoring techniques depending on symptom frequency

\begin{tabular}{|c|c|}
\hline Frequency of symptoms & Suggested ECG monitoring technique \\
\hline $\begin{array}{l}\text { - Daily } \\
\text { - Every 2-3 days } \\
\text { - Every week } \\
\text { - Every month } \\
\text { - Less than once per month }\end{array}$ & $\begin{array}{l}\text { - } 24 \text { h Holter, in-hospital telemetric } \\
\text { monitoring } \\
\text { - } 48-72 \text { h Holter, in-hospital } \\
\text { telemetric monitoring } \\
\text { - } 7 \text { day Holter or external loop } \\
\text { recorder } \\
\text { - } 14-30 \text { days external loop recorder } \\
\text { - Implantable loop recorder }\end{array}$ \\
\hline
\end{tabular}

yield has been calculated to be $43-50 \%$ at 2 years and $80 \%$ at 4 years. ${ }^{5, w 26-w 28}$

- Laboratory testing. The assumption is that provoked abnormalities will have the same mechanism as a spontaneous episode. Tilt table testing and carotid sinus massage are indicated when reflex syncope is suspected in the setting of an atypical (non-diagnostic per se) presentation. EPS is indicated when syncope due to arrhythmia is suspected in patients with previous myocardial infarction, sinus bradycardia, bundle branch block (BBB) or sudden and brief undocumented palpitations. Exercise testing is indicated in patients who experience syncope during or shortly after exertion. Since false positive and negative responses are not uncommon for all these tests, the interpretation of responses requires knowledge of the clinical context in which spontaneous syncope occurred. Knowledge of the rhythm and haemodynamic response during a spontaneous event is the ideal 'gold standard' for evaluation.

The strategy of prolonged monitoring provides reliable evidence of diagnostic accuracy but diagnosis (and therapy) is delayed, often for a long time, until an event can be documented and the recurrent event may cause harm or even death. Conversely, the strategy of laboratory tests has the advantage of an immediate diagnosis and therapy, but is hampered by a significant risk of misdiagnosis.

\subsection{Persistent bradycardia}

This section refers to acquired bradycardia in adults. Refer to section 4.3 for bradycardia in children and in congenital heart disease

\subsubsection{Indications for pacing}

\section{Sinus node disease (Recommendations 1, 2, and 3)}

In general, SB is only an indication for pacing if bradycardia is symptomatic. Symptoms may be present at rest but more frequently develop during exercise. The effect of cardiac pacing on the natural history of bradyarrhythmias comes from old non-randomized studies performed at the beginning of the PM era, which suggested a symptomatic improvement with cardiac pacing. ${ }^{6-9}$ In one RCT, 107 patients with symptomatic sinus node disease (aged $73 \pm 11$ years) were randomized to no treatment, oral theophylline or dualchamber rate-responsive PM therapy, and followed for a mean of $19 \pm 14$ months. During follow-up, the occurrence of syncope and HF were lower in the PM group than in the other groups. Because cardiac pacing is not known to prolong survival in patients with 
sinus node dysfunction, permanent pacing is currently used to relieve symptoms attributed to bradycardia in patients with sinus node disease. If a cause-effect relationship between bradycardia and symptoms is excluded, cardiac pacing is not indicated.

Even if the quality of evidence is modest, there is a strong consensus that patients with symptomatic sinus node disease will benefit from cardiac pacing for symptom relief.

However, in many patients, the clinical manifestations of sinus node disease are more insidious and it is unclear whether symptoms can be attributed to an inadequate heart rate response to activities of daily living. Exercise testing (including cardiopulmonary testing) can be used to assess exercise capacity but the range of heart rates in response to exercise in individuals is wide and therefore standard criteria for chronotropic incompetence are unreliable. A blunted response of heart rate to autonomic blockade with propranolol $(0.2 \mathrm{mg} / \mathrm{kg}$ intravenously) followed by atropine $(0.04 \mathrm{mg} / \mathrm{kg})$ may, on rare occasions, be useful in order to identify patients with chronotropic incompetence and differentiate intrinsic from extrinsic forms of sinus node dysfunction. ${ }^{\text {w29 }}$

Owing to the modest quality of evidence and the large inter-patient variability that make it difficult to establish the nature of symptoms, the usefulness of cardiac pacing in patients with chronotropic incompetence is uncertain, and the decision should be made on a case-by-case basis.

In a controlled, prospective study, ${ }^{\text {w30 }}$ the long-term outcome of 470 patients aged $>60$ years with asymptomatic SB (heart rate $<55$ bpm) was compared with that of 2090 patients without bradycardia. During subsequent mean follow-up of 7.2 years, very few patients in either group needed a PM ( 9 and $5 \%$, respectively). A multivariable analysis showed that bradycardiac patients had a lower mortality, suggesting a protective effect of bradycardia. A low implantation rate, annualized to $<1 \%$ per year, argues against PM implantation in patients with asymptomatic bradycardia.

Even if the quality of evidence is modest, there is a strong consensus that asymptomatic patients with SB do not benefit from cardiac pacing.

\section{Clinical perspectives:}

- Patients with sinus node disease are generally old and frequently have a concomitant heart disease. In these situations, the demonstration of a clear cause-effect relationship between symptoms and sinus node disease is often difficult to achieve.

- It is crucial to distinguish between physiological bradycardia, due to autonomic conditions or training effects, and inappropriate bradycardia that requires permanent cardiac pacing. For example, SB (even when it is $40-50$ bpm while at rest or as slow as $30 \mathrm{bpm}$ while sleeping) is accepted as a physiological finding that does not require cardiac pacing in trained athletes.

- When bradycardia is induced or exacerbated by concomitant drugs affecting sinus node function, drug discontinuation should be considered as an alternative to cardiac pacing. Reducing drug dose, however, may not resolve the bradycardia.

Acquired atrioventricular block (Recommendations 4, 5, and 6) In contrast to SB, AV block may require PM therapy for prognostic reasons and pacing may be indicated in asymptomatic patients.
Although formal RCTs of pacing in patients with third- or seconddegree type 2 AV block have not been performed, several observational studies, performed at the beginning of the PM era, suggest that pacing prevents recurrence of syncope and improves survival in adults. ${ }^{\text {w9-w13 }}$

In second-degree type $1 \mathrm{AV}$ block, the indication for permanent pacing is controversial, unless AV block causes symptoms or the conduction delay occurs at intra- or infra-His levels. ${ }^{\text {w31-w36 }}$ The causeeffect relationship with symptoms is sometimes difficult to determine, especially when symptoms are non-specific and subtle. The progression to complete heart block is likely when there is a wide QRS complex. w35,w37,w38

Even if the quality of evidence is modest, there is a strong consensus that permanent cardiac pacing is indicated in patients with third- or seconddegree type 2 AV block. In patients with type I second-degree AV block, the decision about pacing is controversial, taking into account the severity of symptoms and the risk of progression to complete AV block.

\section{Indication for pacing in patients with persistent bradycardia}

\begin{tabular}{|c|c|c|c|}
\hline Recommendations & Class $^{a}$ & Level $^{\mathrm{b}}$ & Ref. ${ }^{c}$ \\
\hline $\begin{array}{l}\text { I) Sinus node disease. } \\
\text { Pacing is indicated when } \\
\text { symptoms can clearly be } \\
\text { attributed to bradycardia. }\end{array}$ & I & B & $1,6-9$ \\
\hline $\begin{array}{l}\text { 2) Sinus node disease. } \\
\text { Pacing may be indicated when } \\
\text { symptoms are likely to be due } \\
\text { to bradycardia, even if the } \\
\text { evidence is not conclusive. }\end{array}$ & Ilb & C & - \\
\hline $\begin{array}{l}\text { 3) Sinus node disease. } \\
\text { Pacing is not indicated in } \\
\text { patients with SB which is } \\
\text { asymptomatic or due to } \\
\text { reversible causes. }\end{array}$ & III & C & - \\
\hline $\begin{array}{l}\text { 4) Acquired AV block. } \\
\text { Pacing is indicated in patients } \\
\text { with third- or second-degree } \\
\text { type } 2 \mathrm{AV} \text { block irrespective of } \\
\text { symptoms. }\end{array}$ & I & C & - \\
\hline $\begin{array}{l}\text { 5) Acquired AV block. } \\
\text { Pacing should be considered } \\
\text { in patients with second-degree } \\
\text { type I AV block which causes } \\
\text { symptoms or is found to be } \\
\text { located at intra- or infra-His } \\
\text { levels at EPS. }\end{array}$ & Ila & C & - \\
\hline $\begin{array}{l}\text { 6) Acquired AV block. } \\
\text { Pacing is not indicated in } \\
\text { patients with AV block which } \\
\text { is due to reversible causes. }\end{array}$ & III & C & - \\
\hline
\end{tabular}

$\mathrm{AV}=$ atrioventricular; $\mathrm{EPS}=$ electrophysiological study; $\mathrm{SB}=$ sinus bradycardia. ${ }^{\text {a }}$ Class of recommendation.

bLevel of evidence.

${ }^{c}$ Reference(s) supporting recommendation(s). 


\subsubsection{Choice of pacing mode}

Atrial- was compared with ventricular pacing in one trial. ${ }^{10}$ Dual-chamber- was compared with single-chamber (ventricular and atrial) pacing in five multi-centre, parallel, randomized trials, ${ }^{3,11-14}$ one meta-analysis of randomized trials, ${ }^{2}$ and in one systematic review that also included 30 randomized crossover comparisons and four economic analyses. ${ }^{15}$ The results can be summarized as follows (Table 8). Compared with single-chamber-, dual-chamber pacing results in small but potentially important benefits in patients with sinus node disease and/or AV block. No difference in mortality has been observed. Dual-chamber pacing was associated with a lower rate of AF and stroke, but not of HF, although trends in favour of dualchamber pacing were shown in some trials. The effect on AF was more marked in trials including people with sinus node disease. More than a quarter of participants with VVI pacing develop 'pacemaker syndrome', which reduces quality of life. In crossover trials, symptoms of pacemaker syndrome (dyspnoea, dizziness, palpitations, pulsations and chest pain) were reduced by reprogramming to dual-chamber mode. Overall, dual-chamber pacing is associated with better exercise performance compared with fixed-, but not with rate-responsive, VVI pacing. Dual-chamber devices are more expensive owing to the additional lead, longer implantation times and higher risk of complications. Because of the additional clinical consequences of PM syndrome and AF (and its sequelae), the overall cost difference between single and dual systems was not large over a 5 -year period.

An old trial suggested that patients who were paced in AAI mode had lower incidences of AF, thrombo-embolic events, HF, cardiovascular mortality and total mortality, ${ }^{10}$ compared with those paced in VVI mode. However, it remains unclear whether there is any difference between AAIR and dual-chamber pacing (DDDR). ${ }^{\text {w9 }}$ In the recent DANPACE trial, ${ }^{3} 1415$ patients were randomized to AAIR or DDDR pacing and followed for a mean of 5.4 years. There was no difference in all-cause mortality (primary endpoint). AAIR pacing was associated with a higher incidence of paroxysmal AF (HR: 1.27) and a two-fold increased risk of PM re-operation (HR: 1.99). Finally, the disadvantage of AAIR is that AV block develops in $0.6-1.9 \%$ of patients with sinus node disease every year., $30,15, w 40$ These findings support the routine use of DDDR, rather than AAIR, pacing in patients with sinus node disease.

\section{Sinus node disease (Recommendation 7)}

In patients with persistent SB, dual-chamber pacing is the pacing mode of first choice (Figure 3). The results of the DANPACE trial do not support the routine use of AAIR pacing in these patients. ${ }^{3}$ Unnecessary right ventricular (RV) pacing should be systematically avoided in patients with $\mathrm{SB}$, since it may cause $\mathrm{AF}$ and deterioration of HF. ${ }^{16,17}$ However, programming an excessively long AV interval to avoid RV pacing in patients with prolonged AV conduction may be disadvantageous from a haemodynamic point of view by causing diastolic mitral regurgitation, which may cause symptoms and AF. ${ }^{w 41, w 42}$ It is the opinion of this Task Force that in patients with severely reduced left ventricular ejection fraction (LVEF) and indication for pacing for sinus node disease, cardiac resynchronization therapy (CRT) should be considered if a high percentage of ventricular pacing is to be expected (see section 3.4 on CRT).

While there is evidence of superiority of VVIR pacing, compared with VVI pacing, in improving quality of life and exercise capacity (see below), improvements in exercise capacity with DDDR, compared with DDD, have been inconsistent. In two small studies of patients with chronotropic incompetence, ${ }^{w 43, w 44}$ comparing DDD and DDDR pacing, the latter improved quality of life and exercise capacity, but a larger multicentre, randomized trial [ADvanced Elements of Pacing Randomized Controlled Trial (ADEPT) $]^{\mathrm{w} 45}$ failed to show a

Table 8 Outcome of randomized controlled trials of dual-chamber versus ventricular pacing

\begin{tabular}{|c|c|c|c|}
\hline Outcome & References & $\begin{array}{l}\text { Dual-chamber benefit over } \\
\text { ventricular pacing }\end{array}$ & Notes \\
\hline All-cause deaths & $2,11-15$ & No benefit & \\
\hline Stroke, embolism & $2,11-15$ & $\begin{array}{l}\text { Benefit (in meta-analysis only, not in } \\
\text { single trial) }\end{array}$ & HR 0.80.' ${ }^{12}$ Benefit higher in SSS. \\
\hline Atrial fibrillation & $2,11-15$ & Benefit & HR $0.8 I^{12}$ and $0.76 . .^{13}$ Benefit higher in SSS. \\
\hline HF, hospitalization for HF & $2,11,12,14,15$ & No benefit & \\
\hline Exercise capacity & 15 & Benefit & $\begin{array}{l}\text { Overall standardized mean improvement of } 35 \% \text {. } \\
\text { Not significant compared to VVIR. }\end{array}$ \\
\hline Pacemaker syndrome & $11-13,15$ & Benefit & Documented in up to $25 \%$ of VVI patients. \\
\hline Functional status & $\mathrm{II}, 12,15$ & No benefit & \\
\hline Quality of life & $11-13,15$ & Variable & $\begin{array}{l}\text { Consistent direction of effect on quality of life, but the } \\
\text { size cannot be estimated with confidence. }\end{array}$ \\
\hline Complications & $2,11-13,15$ & $\begin{array}{l}\text { More complications with } \\
\text { dual-chamber }\end{array}$ & $\begin{array}{l}\text { Higher rate of lead dislodgment ( } 4.25 \text { vs. I. } 4 \% \text { ) and } \\
\text { inadequate pacing (I.3 vs. } 0.3 \%) \text {. }\end{array}$ \\
\hline
\end{tabular}




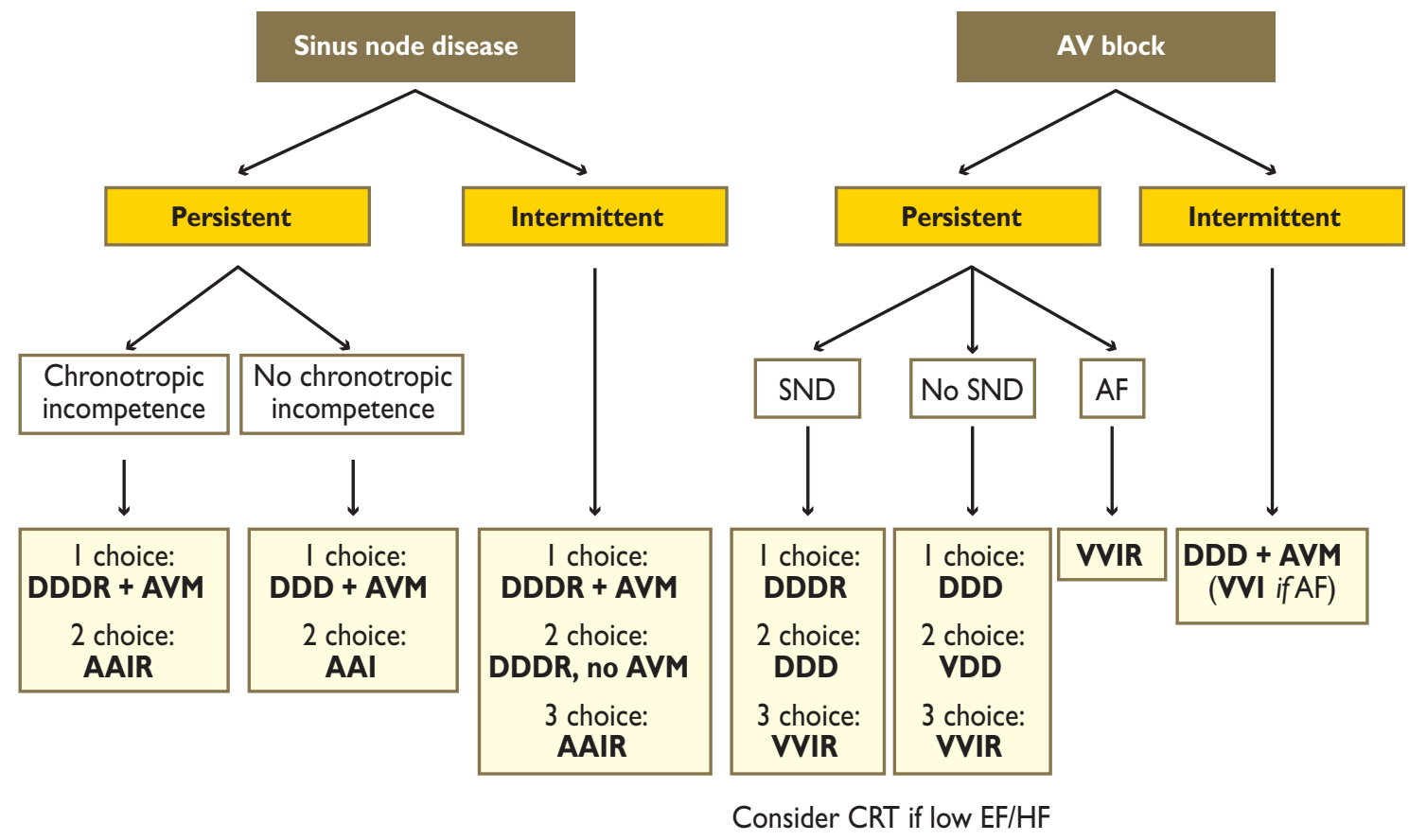

Figure 3 Optimal pacing mode in sinus node disease and $A V$ block. $A F=$ atrial fibrillation; $A V=$ atrioventricular; $A V M=A V$ delay management, i.e. to prevent unnecessary right ventricular pacing by means of manual optimization of $A V$ interval or programming of $A V$ hysteresis; $S N D=$ sinus node disease.

difference in patients with modest blunted heart rate response to exercise.

There is evidence of superiority of dual-chamber pacing over ventricular pacing. The evidence is stronger for patients with sinus node disease. Further research is very unlikely to change our confidence in the estimate of effect. The evidence of superiority of dual-chamber vs. single-chamber atrial pacing is weaker.

Whilst there is sufficient evidence of superiority of rate-responsive ventricular pacing, compared with fixed-rate pacing, in improving quality of life and exercise capacity, this evidence is much weaker in dualchamber pacing with or without rate-response features.

\section{Clinical perspectives:}

- In patients with sinus node disease, dual-chamber pacing confers a modest reduction in AF and stroke, but not in hospitalization for heart failure or death compared with ventricular pacing.

- Dual-chamber pacing reduces the risk of PM syndrome which occurs in more than a quarter of patients with either sinus node disease and AV block. PM syndrome is associated with a reduction in quality of life and often justifies the preference for dual-chamber pacing. Even if it is a softer endpoint, PM syndrome is associated with a reduction in quality of life and justifies the preference for dual-chamber pacing when reasonable.

\section{Acquired atrioventricular block (Recommendation 8)}

Large randomized parallel trials were unable to show a superiority of dual-chamber pacing over ventricular pacing with regard to hard clinical endpoints of mortality and morbidity. ${ }^{2,11,13,14}$ The benefit of dual-chamber over ventricular pacing is mostly due to the avoidance of PM syndrome, which occurs in more than a quarter of patients with AV block, and to an improved exercise capacity. ${ }^{15}$ This effect was consistently observed in 26 crossover trials. ${ }^{15}$ Even if it is a softer endpoint, PM syndrome is associated with reduction in quality of life and justifies the preference for dual-chamber pacing when reasonable (Figure 3).

There is strong evidence of superiority of dual-chamber pacing over ventricular pacing, limited to symptom improvement. Conversely, there is strong evidence of non-superiority with regard to survival and morbidity. In consequence, the indication for dual-chamber mode is weak and the decision on pacing mode should be made on an individual basis, taking into consideration the increased complication risk and costs of dualchamber pacing. Further research is very unlikely to change our confidence in the estimate of effect.

\section{Clinical perspectives:}

- In patients with AV block, dual-chamber pacing does not reduce morbidity (hospitalization, HF) or mortality, compared with ventricular pacing.

- In patients with AV block (including those with SB and long PR interval) who will probably require a high percentage of ventricular pacing, CRT should be considered if clinical symptoms of HF and a severely reduced LVEF are present (see section 3.4 on CRT).

- Advice for follow-up: it is advisable that mode-switch algorithm be activated; evolution of AF during follow-up should be assessed by the diagnostics of the device with a view to anticoagulant therapy when 
necessary; rate-response function should be properly programmed at implantation and reassessed during follow-up; finally, the percentage of ventricular pacing should be assessed at each follow-up, in order to minimize it as much as possible.

\section{Permanent atrial fibrillation and atrioventricular block (Recommendation 9)}

Rate-responsive pacing is associated with better exercise performance, improved daily activities, decrease of symptoms of shortness of breath, chest pain and palpitations and improved quality of life, compared with fixed-rate pacing. ${ }^{\text {w6-w48 }}$ Therefore, rate-adaptive pacing is the pacing mode of first choice and fixed-rate $\mathrm{VVI}$ pacing should be abandoned in patients with permanent AF and AV block (Figure 4). It is the opinion of this Task Force that the minimum rate should be programmed higher (e.g. $70 \mathrm{bpm}$ ) than for SR patients in an attempt to compensate for loss of active atrial filling and the maximum sensor rate should be programmed restrictively (e.g. 110-120 bpm), in order to avoid 'overpacing', i.e. pacing with a heart rate faster than necessary, which can be symptomatic, especially in patients with coronary artery disease. However, in a small study, ${ }^{\text {49 }}$ rate-responsive pacing was safe and effective in patients with angina pectoris, without an increase in subjective or objective signs of ischaemia.

\section{Choice of pacing mode/programming in patients with persistent bradycardia}

\begin{tabular}{|c|c|c|c|}
\hline Recommendations & Class $^{a}$ & Level $^{\mathrm{b}}$ & Ref. ${ }^{C}$ \\
\hline $\begin{array}{l}\text { 7) Sinus node disease. } \\
\text { 7A) Dual-chamber PM } \\
\text { with preservation of } \\
\text { spontaneous AV conduction } \\
\text { is indicated for reducing } \\
\text { the risk of AF and stroke, } \\
\text { avoiding PM syndrome and } \\
\text { improving quality of life. }\end{array}$ & I & $\begin{array}{c}\begin{array}{c}\text { A } \\
\text { (vs. VVI) }\end{array} \\
\text { B } \\
\text { (vs. AAI) }\end{array}$ & $\begin{array}{c}2,3,11-13 \\
15-17\end{array}$ \\
\hline $\begin{array}{l}\text { 7B) Rate response features } \\
\text { should be adopted for } \\
\text { patients with chronotropic } \\
\text { incompetence, especially if } \\
\text { young and physically active. }\end{array}$ & Illa & C & - \\
\hline $\begin{array}{l}\text { 8) Acquired AV block. } \\
\text { In patients with sinus rhythm, } \\
\text { dual-chamber PM should be } \\
\text { preferred to single chamber } \\
\text { ventricular pacing for avoiding } \\
\text { PM syndrome and improving } \\
\text { quality of life. }\end{array}$ & Ila & $\mathbf{A}$ & $\begin{array}{l}2,11, \\
13-15\end{array}$ \\
\hline $\begin{array}{l}\text { 9) Permanent AF and } \\
\text { AV block. } \\
\text { Ventricular pacing with } \\
\text { rate-response function is } \\
\text { recommended. }\end{array}$ & I & C & - \\
\hline
\end{tabular}

$\mathrm{AF}=$ atrial fibrillation; $\mathrm{AV}=$ atrioventricular; $\mathrm{PM}=$ pacemaker

${ }^{a}$ Class of recommendation.

bLevel of evidence.

'Reference(s) supporting recommendation(s).

\subsection{Intermittent (documented) bradycardia}

\subsubsection{Indications for pacing}

\section{Sinus node disease, including brady-tachy form (Recommenda- tions 1 and 4)}

Schematically, there are two clinical features of intermittent bradycardia in patients affected by intrinsic sinus node disease, in which cardiac pacing is indicated: (i) documented symptomatic intermittent sinus arrest or sino-atrial block in patients with asymptomatic, permanent, mild (i.e. heart rate $40-50$ bpm) SB and (ii) prolonged sinus pause following the termination of tachycardia in the bradytachy syndrome. In both, the underlying mechanism is the abnormally prolonged time needed for recovery of automaticity by a diseased sinus node. Prolonged pauses (i.e. $>3$ s) typically cause (pre-) syncope, which is the reason for cardiac pacing.

No trial has specifically addressed the role of cardiac pacing in these two settings, since these patients were part of the larger population of patients affected by sinus node disease (see section 3.1). ${ }^{1,6-9}$ However, syncope and supraventricular tachyarrhythmias were very frequent in trials of patients with sinus node disease. For example, syncope was present in $60 \%$ of patients enrolled in the Effects of Oral THEOphylline and of Permanent Pacemaker on the Symptoms and Complications of Sick Sinus Syndrome (THEOPACE) trial and in $50 \%$ of patients enrolled in the DANPACE trial. ${ }^{1,3}$ Supraventricular tachyarrhythmias were present in $53 \%$ of the patients enrolled in the MOde Selection Trial in Sinus-Node Dysfunction (MOST) trial and a brady-tachy form of SSS was diagnosed in $38 \%$ of the patients enrolled in the THEOPACE trial. ${ }^{1,12}$

\section{When the correlation between symptoms and ECG is established, there is general consensus that cardiac pacing is effective and useful for symptom relief.}

In the absence of such correlation, the mechanism of undocumented intermittent symptoms (e.g. syncope) in patients with permanent mild (i.e. 40-50 bpm) SB, itself asymptomatic, remains uncertain as other competitive causes (i.e. a disturbance of the autonomic nervous system) often play an important role. ${ }^{\text {w23,w24 }}$ In patients with sinus node disease and syncope, carotid sinus hypersensitivity and a positive response to tilt are present in up to $50 \%$ of patients (see also section 3.3). Thus, an increased susceptibility to neurallymediated bradycardia/hypotension is often the cause of syncope. A reflex mechanism of syncope fits well with the unpredictable natural history of syncopal recurrences and may, in part, explain why syncope recurs in about $20 \%$ of SSS patients during long-term follow-up, despite adequate pacing. ${ }^{w 50}$ Some small studies suggested that a very prolonged SNRT (i.e. $>3 \mathrm{sec}$ ) or a very prolonged, corrected SNRT (i.e. $\geq 800 \mathrm{~ms}$ ) indicate a likely bradyarrhythmic mechanism of syncope, ${ }^{w 51-w 53}$ but the precise role of EPS for the selection of candidates for cardiac pacing has never been established. EPS is currently performed in a few doubtful cases. This Task Force recognizes that there is an occasional need, in practice, to make a therapeutic decision with weaker diagnostic criteria. Physicians should be aware that effectiveness of therapy is not well documented in such cases. From a practical perspective, cardiac pacing may be a reasonable solution in patients affected by sinus node disease, who have the documentation of an asymptomatic ventricular pause $>3 \mathrm{sec}$ 
(with exceptions for young trained persons, during sleep and medicated patients), when a competitive diagnosis, i.e. hypotension, can be ruled out.

When the correlation between symptoms and ECG is not established, cardiac pacing may be reasonable in patients with intrinsic sinus node disease, syncope and documentation of asymptomatic pause/s.

\section{Intermittent/paroxysmal atrioventricular block, including atrial fibrillation with slow ventricular conduction (Recommenda- tions 2 and 4)}

Intermittent/paroxysmal AV block that occurs in patients with underlying heart disease and/or BBB is usually regarded as a manifestation of intrinsic disease of the AV conduction system (Stokes-Adams). Welldefined clinical and electrophysiological features allow intrinsic AV block to be differentiated from the other known form of block, namely, vagal (extrinsic) and idiopathic AV block. ${ }^{\text {w20,w21 Documenta- }}$ tion of infra-Hisian block by EPS or the documentation of initiation of the block by atrial or ventricular premature beats, or increased heart rate (tachy-dependent AV block) or decreased heart rate (bradydependent AV block), support a diagnosis of intrinsic AV block. In these cases, the cardiac rhythm may become dependent on subsidiary (often unreliable) pacemaker sites. Syncope occurs due to the often-long delay before these pacemaker sites take over. In addition, these subsidiary pacemaker sites have relatively slow rates (typically 25-40 bpm); consequently, syncope or pre-syncope may be due to inadequate cerebral perfusion. In patients with documented third- or second-degree AV block due to intrinsic disease of the AV conduction system, symptom-rhythm correlation is less important than in sinus node disease, because there is general consensus that pacing prevents recurrence of syncope and may improve survival.

Indications for permanent pacing in intermittent high-degree AV block are similar to those in persistent AV block (see section 3.1).

There is general consensus that cardiac pacing is indicated in patients with intrinsic intermittent AV block, even in the absence of documentation of symptom-ECG correlation.

\section{Intermittent bradycardia and asystole in patients with reflex syncope (Recommendations 3 and 4)}

Differentiation between the often benign and reversible causes of extrinsic (reflex) sinus arrest and AV block from intrinsic forms is of practical importance because the benefit of permanent cardiac pacing is less established. The International Study on Syncope of Unexplained Etiology (ISSUE) classification has some pathophysiological implications that are helpful in distinguishing between extrinsic and intrinsic forms (Web Table 9). ${ }^{\text {w54 }}$ In observational studies, cardiac pacing reduced syncope burden in patients with documented asystolic syncope, but did not prevent all syncopal events. ${ }^{5,18}$ In the recent randomized, double-blind ISSUE 3 trial, ${ }^{19} 511$ patients $\geq 40$ years with recurrent reflex syncopes received an ILR; 89 of these had documentation of syncope with $\geq 3 \mathrm{sec}$ asystole or $\geq 6 \mathrm{sec}$ asystole without syncope within $12 \pm 10$ months, and met criteria for PM implantation; 77 of 89 patients were randomly assigned to dual-chamber pacing with rate drop response or to sensing only. The data were analysed on an intention-to-treat principle. During follow-up, the 2-year estimated syncope recurrence rate was $57 \%$ with PM OFF and $25 \%$ with $\mathrm{PM} O N$ (log-rank $P=0.039$ ). The risk of recurrence was reduced by $57 \%$. The ISSUE studies focused on subjects affected by reflex syncope with a relatively high mean age, a history of recurrent syncope beginning in middle or older age and frequent injuries probably due to lack of a prodrome. The ISSUE-like patients match those defined by the 2009 ESC Guidelines as high-risk or high-frequency. Young patients, who usually have a more prolonged prodrome before loss of consciousness, were not included in the ISSUE population.

When the correlation between symptoms and ECG is established, there is sufficient evidence that dual-chamber cardiac pacing is effective and useful for prevention of syncopal recurrences and reduction of syncope burden in patients $\geq 40$ years with the clinical features of those used in the ISSUE studies.

With the increasing use of prolonged monitoring techniques, it is not unusual to document even long asymptomatic pauses. Often, patients are unable to ascertain whether they had had any symptom at the time of ECG documentation of an asystolic pause. A typical situation is that of long pauses occurring during sleep. ${ }^{w 55, w 56}$ Patients give a history of waking up with abdominal discomfort or nightmares. In the absence of a cause-effect relationship, the meaning of asymptomatic pauses in patients with a clinical diagnosis of reflex syncope is uncertain. Few studies have found a good intrapatient correlation between non-syncopal and syncopal episodes. $^{\text {w57,w58 }}$ This issue is of practical importance, since a good correlation with the index syncope would allow the use of non-syncopal documented events as surrogate endpoints, predictive of the mechanism of syncope. The length of the pause is of major importance. Under physiological circumstances, asystole of $3 \mathrm{sec}$ (and the corresponding heart rate of $<20 \mathrm{bpm}$ ) is a rarity. ${ }^{\text {w59 }}$ But this per se does not necessarily define a clinical disorder. The current $3 \mathrm{sec}$ criterion is an arbitrary clinical observation and has a low specificity. Pathophysiological reasoning and clinical observations suggest that a pause of $\geq 6 \mathrm{sec}$ is necessary to cause symptoms. ${ }^{w 60, w 61}$ In ISSUE-2, ${ }^{w 62}$ the average pause at time of documented syncope was $9 \mathrm{sec}$ (range $8-18 \mathrm{sec}$ ). In the recent ISSUE 3 trial, $^{19}$ the patients with syncope had an asystolic pause of $12 \pm 10 \mathrm{sec}$ and those without syncope an asystolic pause of $10 \pm 6 \mathrm{sec}$.

In patients with a clinical diagnosis of reflex syncope and asymptomatic pause $>6 \mathrm{sec}$, there is weak evidence that cardiac pacing may be effective and useful for the reduction of syncopal recurrences.

Finally, there are no data regarding the effect of cardiac pacing in syncopal patients with documentations, at the time of syncope, of intermittent bradycardia without asystolic pauses. Therefore, no recommendations can be made.

\section{Clinical perspectives:}

- In patients with reflex syncope, cardiac pacing should be the last choice and should only be given to highly selected patients, i.e. those of relatively old age, affected by severe forms of reflex syncope with a history of recurrent syncope and frequent injuries, probably due to lack of prodromal symptoms. The fact that pacing is effective in some patients with reflex syncope does not mean that it is also always necessary. It must be emphasized that the decision to implant a PM needs to be undertaken 
in the clinical context of a benign condition (in terms of mortality), which frequently affects young patients.

- Establishing a relationship between symptoms and bradycardia should be the goal of the clinical evaluation of patients with unexplained syncope and normal baseline ECG. Monitoring should be prolonged until such a goal is achieved. In real-world practice, there is an occasional need to make a therapeutic decision with weaker diagnostic criteria. In such circumstances, it is the opinion of this Task Force that an asymptomatic pause $>6$ sec is probably associated with benefit of cardiac pacing. Cardiac pacing should be delayed in patients with asymptomatic pauses $<6$ sec and monitoring continued.

\section{Indication for pacing in intermittent documented bradycardia}

\begin{tabular}{|c|c|c|c|}
\hline Recommendations & Class $^{a}$ & Level $^{b}$ & Ref. $^{c}$ \\
\hline $\begin{array}{l}\text { I) Sinus node disease } \\
\text { (including brady-tachy } \\
\text { form). } \\
\text { Pacing is indicated in patients } \\
\text { affected by sinus node disease } \\
\text { who have the documentation } \\
\text { of symptomatic bradycardia } \\
\text { due to sinus arrest or sinus- } \\
\text { atrial block. }\end{array}$ & I & B & $1,6-9$ \\
\hline $\begin{array}{l}\text { 2) Intermittent/ } \\
\text { paroxysmal AV block } \\
\text { (including AF with slow } \\
\text { ventricular conduction). } \\
\text { Pacing is indicated in patients } \\
\text { with intermittent/paroxysmal } \\
\text { intrinsic third- or second- } \\
\text { degree AV block. }\end{array}$ & I & C & - \\
\hline $\begin{array}{l}\text { 3) Reflex asystolic } \\
\text { syncope. } \\
\text { Pacing should be considered } \\
\text { in patients } \geq 40 \text { years with } \\
\text { recurrent, unpredictable reflex } \\
\text { syncopes and documented } \\
\text { symptomatic pause/s due to } \\
\text { sinus arrest or AV block or the } \\
\text { combination of the two. }\end{array}$ & Ila & $\mathbf{B}$ & $5,18,19$ \\
\hline $\begin{array}{l}\text { 4) Asymptomatic pauses } \\
\text { (sinus arrest or AV } \\
\text { block). } \\
\text { Pacing should be considered } \\
\text { in patients with history of } \\
\text { syncope and documentation } \\
\text { of asymptomatic pauses }>6 \mathrm{~s} \\
\text { due to sinus arrest, sinus-atrial } \\
\text { block or AV block. }\end{array}$ & Ila & C & - \\
\hline $\begin{array}{l}\text { 5) Pacing is not indicated } \\
\text { in reversible causes of } \\
\text { bradycardia. }\end{array}$ & III & C & - \\
\hline
\end{tabular}

$\mathrm{AF}=$ atrial fibrillation; $\mathrm{AV}=$ atrioventricular.

${ }^{\mathrm{a}}$ Class of recommendation.

bLevel of evidence.

'Reference(s) supporting recommendation(s).

\subsubsection{Choice of pacing mode}

In intermittent bradycardia, pacing may be required only for short periods of time. In this situation, the benefits of bradycardia and pause prevention must be weighed against the detrimental effects of permanent pacing, particularly pacing-induced HF. Patients should not be subjected to permanent ventricular stimulation. Therefore, manual adaptation of AV interval (up to $250 \mathrm{~ms}$ ) or programming of AV hysteresis preventing unnecessary RV pacing plays a particularly important role in this patient group ${ }^{16,17}$ (see also section 2.1). The evidence of benefit is stronger for sinus node disease.

In the absence of studies including patients with intermittent bradycardia only, this Task Force is unable to make an evidence-based specific recommendation on the choice of pacing mode (VVI vs. DDD). Therefore, we refer to the recommendations for permanent bradycardia. Figure 3 reflects the opinion of the members of the Task Force. The main reason for the preference for dual-chamber over single-chamber ventricular pacing is the risk of PM syndrome caused by this latter modality. PM syndrome is particularly important in patients with reflex intermittent bradycardia. Consistent with patients affected by carotid sinus syndrome or tilt-induced reflex syncope (see section 2.4), adequate rate hysteresis should be programmed in order to allow spontaneous sinus rate to emerge and to restrict pacing to the short period of time in which reflex bradycardia occurs.

In the ISSUE 2 and ISSUE 3 trials, DDD pacing with rate hysteresis (with specific rate-drop algorithm) was used in patients with documented reflex asystolic syncope, but these trials did not consider a comparison with other pacing modalities. ${ }^{5,19}$ In all trials of cardiac pacing in tilt-positive vasovagal patients, dual-chamber pacing was used - with a rate drop response feature of the PM that instituted rapid DDD pacing if the device detected a rapid decrease in heart rate-but no comparison with conventional dual-chamber pacing has ever been made. ${ }^{20-24}$

\begin{tabular}{l} 
Choice of pacing mode \\
\begin{tabular}{|l|c|c|c|}
\hline Recommendations & Class $^{\text {a }}$ & Level $^{\text {b }}$ & Ref. $^{\mathrm{C}}$ \\
\hline $\begin{array}{l}\text { 6) Intermittent } \\
\text { documented bradycardia. } \\
\text { Preservation of spontaneous } \\
\text { AV conduction is } \\
\text { recommended. }\end{array}$ & I & B & I6, I7 \\
\hline $\begin{array}{l}\text { 7) Reflex asystolic } \\
\text { syncope. } \\
\text { Dual-chamber pacing with } \\
\text { rate hysteresis is the preferred } \\
\text { mode of pacing in order to } \\
\text { preserve spontaneous sinus } \\
\text { rhythm. }\end{array}$ & & & \\
\hline
\end{tabular} \\
\hline
\end{tabular}

$\mathrm{AV}=$ atrioventricular

${ }^{\mathrm{a}}$ Class of recommendation.

bLevel of evidence.

${ }^{\mathrm{c}}$ Reference(s) supporting recommendation(s). 


\section{Section 2.4 Suspected (undocumented) bradycardia}

\subsubsection{Bundle branch block}

The diagnostic work-up of the patient with BBB and syncope should follow the 2009 ESC Guidelines for the diagnosis and management of syncope. ${ }^{w 25}$ The presence of BBB suggests that the cause of syncope may be complete heart block. Nevertheless, less than half of the patients with BBB and syncope have a final diagnosis of cardiac syncope. A similar percentage have a final diagnosis of reflex syncope and, in about $15 \%$, the cause remains unexplained at the end of a complete work-up. ${ }^{25, w 63-w 65}$ This issue has posed many problems in the past. Today, due to the advent of the ILR, two studies specifically addressed the problem of BBB and syncope. Consistent with a systematic diagnostic approach depicted in Figure 4, the ISSUE 1 study and the Bradycardia detection in Bundle Branch Block (B4) study, ${ }^{25, w 64}$ which were performed in patients with normal or preserved systolic function ( $E F>35 \%$ ), showed that about half of patients required pacing and that it is safe to wait until the correct diagnosis has been made. In the B4 study, ${ }^{25}$ of the 215 patients in whom diagnosis was achieved at phases I or II (i.e. during the initial evaluation or by means of carotid sinus massage or EPS) and who were treated according to the findings, a syncopal recurrence was observed in 7\%; in contrast, syncope recurred in 33\% of 52 untreated patients (during ILR observation) $(P=0.001)$. Mortality was $6 \%$ during 19 months of follow-up, mostly non-arrhythmic; compared with those who had ILR, there was no difference in mortality rate between patients diagnosed at phase I or II, who received appropriate treatment.

Although syncope is not associated with an increased incidence of sudden death in patients with preserved cardiac function, a high incidence of total deaths (about one third sudden) was observed in patients with $\mathrm{BBB}$, especially those with $\mathrm{HF}$, previous myocardial infarction or low ejection fraction (EF). ${ }^{w 66-w 68}$ Indeed, the high totaland sudden mortality seems mainly related to underlying structural heart disease and ventricular tachyarrhythmias. Unfortunately, ventricular programmed stimulation does not seem to be able to correctly identify these patients and the finding of inducible ventricular arrhythmia should therefore be interpreted with caution. ${ }^{\text {w66,w67 }}$ Therefore, an implantable cardioverter defibrillator (ICD) or a cardiac resynchronization therapy and defibrillator (CRT-D) should be considered in patients with $\mathrm{BBB}$ and congestive HF, previous myocardial infarction or $\mathrm{EF}<35 \%$, according to the recommendations of current ESC Guidelines for ICD (see also section 3.2). ${ }^{\text {w69 }}$

\section{Bundle branch block, unexplained syncope and abnormal electrophysiological study (Recommendation 1)}

Electrophysiological assessment includes measurement of the Hisventricular $(\mathrm{HV})$ interval at baseline, with stress by incremental atrial pacing and with pharmacological provocation (ajmaline, procainamide or disopyramide). The prognostic value of the HV interval was prospectively studied by Scheinman et al.; ${ }^{26}$ the progression rate to AV block at 4 years was $\leq 4 \%$ in patients with $\mathrm{HV}$ interval $<70 \mathrm{~ms}, 12 \%$ in those with HV interval of $70-100 \mathrm{~ms}$ and $24 \%$ in those with HV interval $>100 \mathrm{~ms}$. The development of intra- or infra-His block at incremental atrial pacing is highly predictive of impending AV block, but is rarely observed and has low sensitivity. For example, in the study by Gronda et al. on 131 patients, ${ }^{27} \mathrm{HV}$ prolongation of $>10 \mathrm{~ms}$ was observed in $6 \%$ and induction of second degree AV block in 5\% of cases. Complete AV block developed in $40 \%$ of these patients during a mean follow-up of 42 months. In five studies evaluating the diagnostic value of pharmacological stress testing for a total of 333 patients, ${ }^{27-31}$ high-degree AV block was induced in 50 (15\%) of the patients. During

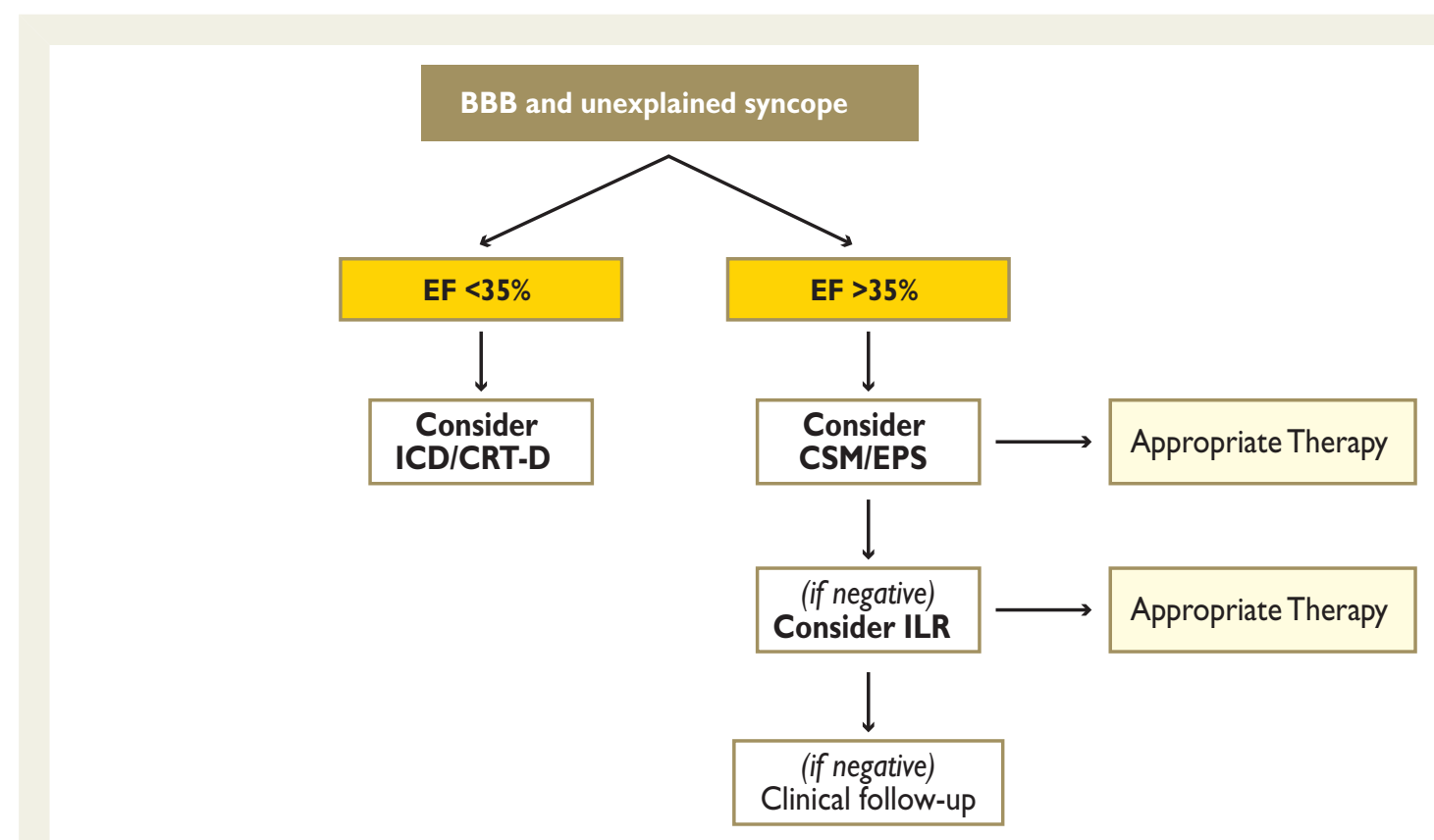

Figure 4 Therapeutic algorithm for patients presenting with unexplained syncope and bundle branch block (BBB). CRT-D = cardiac resynchronization therapy and defibrillator; $C S M=$ carotid sinus massage; $E F=$ ejection fraction; $E P S=$ electrophysiological study; $I C D=$ implantable cardioverter defibrillator; ILR = implantable loop recorder. 
the follow-up of 24-63 months, $68 \%$ (range $43-100$ ) of these patients developed spontaneous AV block. By combining the abovementioned parts of the electrophysiological protocol, a positive EPS yielded a positive predictive value as high as $\geq 80 \%$ to identify the patients who will develop AV block; ${ }^{27,28}$ this finding has been indirectly confirmed by the recent $\mathrm{B} 4$ study, ${ }^{25}$ that showed a significant reduction in syncopal recurrences in patients with positive EPS treated with PM, compared with a control group of untreated patients with negative EPS. With respect to ESC Guidelines on Syncope, ${ }^{\text {w25 }}$ these results justify a recommendation upgrade from class lla to class I.

Thus, in patients with unexplained syncope and bifascicular block, EPS is highly sensitive in identifying patients with intermittent or impending high-degree AV block, though a negative electrophysiological investigation cannot rule out intermittent/paroxysmal AV block as the cause of syncope. Indeed, in patients with negative electrophysiological studies, intermittent or stable AV block was still documented by ILR in about $50 \%$ of cases. ${ }^{25, w 64}$

Even if the quality of evidence is moderate, there is a strong consensus that patients with positive EPS benefit from pacing therapy. However, the benefit should be weighed against the risk and cost of an invasive procedure.

\section{Alternating bundle branch block (Recommendation 2)}

Alternating BBB (also known as bilateral BBB) refers to situations in which clear ECG evidence for block in all three fascicles is manifested on successive ECGs. Examples are right bundle branch block (RBBB) and left bundle branch block (LBBB) on successive ECGs or RBBB with associated left anterior fascicular block on one ECG and associated left posterior fascicular block on another ECG. Patients with ECG documentation of alternating BBB are rare. There is general consensus - even if based on anecdotal cases - that these patients progress rapidly toward AV block. Therefore a PM is usually implanted as soon as the alternating BBB is detected, even in the absence of a history of syncope. ${ }^{w 70-w 73}$

Even if the quality of evidence is modest, there is a strong consensus that patients with alternating BBB will benefit from cardiac pacing.

\section{Bundle branch block, unexplained syncope and non-diagnostic investigations (Recommendation 3)}

The ILR experience ${ }^{25, w 64}$ showed that only about half of patients with unexplained syncope and BBB had a documentation of AV block during the period of observation. In a recent randomized, singleblinded study, ${ }^{32} 51$ patients with bifascicular block assigned to active DDD $60 \mathrm{bpm}$ pacing were compared with 49 patients with bifascicular block assigned to inactive pacing (DDI 30 bpm). At 2 years, syncope or pre-syncope recurred in $45 \%$ of patients in the control group vs. $25 \%$ of patients in the treatment group (hazards ratio, $0.43 ; P=0.005)$. Overall, a bradycardia was documented in only 14 patients ( 10 symptomatic AVB, 2 brady-tachy, 1 sinus bradycardia, and 1 permanent $A F$ with slow ventricular response), accounting for an overall incidence of $7.4 \%$ per year. Albeit the study showed that cardiac pacing was able to achieve a significant reduction in symptoms, only one out of five patients actually had a benefit and symptoms persisted in a quarter of them.

Thus the decision to implant a PM is determined by an individual risk-benefit evaluation. There are subsets of patients who might receive a favourable cost-effective benefit from this strategy; for example, old patients with unpredictable (no- or very short prodromes) and recurrent syncope that expose them to high risk of traumatic recurrences.

The evidence of efficacy of empirical pacing strategy is weak and the estimate of benefit uncertain.

\section{Asymptomatic bundle branch block (Recommendation 4)}

Permanent PM implantation is not indicated for BBB without symptoms - with the exception of alternating BBB - because only a small minority of these patients will develop AV block (1-2\% per year) and because cardiac pacing has not been proven to reduce mortality. $26,33,34$

There is sufficient evidence and strong consensus that pacing is not indicated in patients with asymptomatic BBB.

\section{Clinical perspectives:}

- Less than half of the patients with BBB and syncope have a final diagnosis of cardiac syncope, albeit the probability is different among different type of BBB. We recommend any useful investigation (e.g. carotid sinus massage, EPS and ILR) to provoke/document the mechanism of syncope before deciding to implant a PM or selecting the correct therapy.

- Old patients with BBB and unexplained syncope after a reasonable work-up might benefit from empirical PM, especially if syncope is unpredictable (with no- or short prodrome) or has occurred in supine position or during effort.

\section{Indication for cardiac pacing in patients with BBB}

\begin{tabular}{|c|c|c|c|}
\hline Recommendations & Class $^{a}$ & Level $^{\text {b }}$ & Ref. $^{c}$ \\
\hline $\begin{array}{l}\text { I) BBB, unexplained } \\
\text { syncope and abnormal } \\
\text { EPS. } \\
\text { Pacing is indicated in patients } \\
\text { with syncope, BBB and positive } \\
\text { EPS defined as HV interval } \\
\text { of } \geq 70 \mathrm{~ms} \text {, or second- or } \\
\text { third-degree His-Purkinje } \\
\text { block demonstrated during } \\
\text { incremental atrial pacing } \\
\text { or with pharmacological } \\
\text { challenge. }\end{array}$ & I & B & 25,31 \\
\hline $\begin{array}{l}\text { 2) Alternating BBB. } \\
\text { Pacing is indicated in patients } \\
\text { with alternating BBB with or } \\
\text { without symptoms. }\end{array}$ & I & C & - \\
\hline $\begin{array}{l}\text { 3) BBB, unexplained } \\
\text { syncope non diagnostic } \\
\text { investigations. } \\
\text { Pacing may be considered } \\
\text { in selected patients with } \\
\text { unexplained syncope and BBB. }\end{array}$ & Ilb & B & 32 \\
\hline $\begin{array}{l}\text { 4) Asymptomatic BBB. } \\
\text { Pacing is not indicated for BBB } \\
\text { in asymptomatic patients. }\end{array}$ & III & $\mathbf{B}$ & $26,33,34$ \\
\hline
\end{tabular}

$\mathrm{BBB}=$ bundle branch block; EPS = electrophysiological study

${ }^{a}$ Class of recommendation.

bLevel of evidence.

${ }^{\mathrm{c}}$ Reference(s) supporting recommendation(s). 
- In patients with BBB and severe systolic left ventricular (LV) dysfunction an implantable cardioverter defibrillator (ICD) or CRT-D should be considered instead of PM (refer to section 4.1).

- In patients with previous myocardial infarction and BBB, EPS with programmed ventricular stimulation is particularly advisable. If a sustained ventricular tachycardia is induced, an ICD must be implanted instead of a PM.

\subsubsection{Reflex syncope}

The diagnostic work-up of the patient with syncope should follow that recommended by the 2009 ESC Guidelines for diagnosis and management of syncope. ${ }^{\text {w25 }}$ Often, reflex syncope has an 'atypical' presentation. The term 'atypical form' is used to describe those situations in which reflex syncope occurs with uncertain —or even apparently absent-triggers. The diagnosis then relies less on historytaking alone and more on the exclusion of other causes of syncope (absence of structural heart disease) and on reproducing similar symptoms with carotid sinus massage and tilt-table testing.

\section{Carotid sinus syncope}

This syndrome is currently defined as syncope with carotid sinus massage yielding either asystole of $>3 \mathrm{sec}$ or fall in systolic blood pressure of $>50 \mathrm{mmHg}$, or both, and reproduction of the spontaneous syncope. In order to be as diagnostic as possible, massage is recommended in both supine and erect positions. There is, however, a case for adjusting the diagnostic criteria, particularly in terms of the duration of asystole to be classed as positively diagnostic to $>6 \mathrm{sec}^{\mathrm{w} 74}$ Recommendations for pacing in carotid sinus syncope are confirmed as before, ${ }^{\text {w25 }}$ but a full $10 \mathrm{sec}$ of massage is required; massage is to be performed supine and erect and pacing (dual chamber) is indicated when $>6 \mathrm{sec}$ asystole occurs with reproduction of syncope. The decision to implant a PM should be kept in the context of a relatively benign condition, with the aim of preventing traumatic recurrences, which are frequent in old patients with recurrent carotid sinus syncope. ${ }^{\text {w75 }}$

\section{Carotid sinus syncope (Recommendation 1)}

The evidence supporting the benefit of cardiac pacing in patients affected by carotid sinus syncope is limited to a few small controlled trials and retrospective observational studies. In a review of the literature, ${ }^{35}$ the natural history was analysed of 305 patients and the effect of cardiac pacing of another 601 patients affected by severe recurrent syncope, who were followed-up to 5 years (Figure 5).

The studies were largely heterogeneous as regards selection of the patients, duration and position (supine or standing) of the carotid sinus massage, criteria for identification of mixed form and different modes of pacing (single vs. dual-chamber). In general, with pacing, the syncopal recurrence rate during follow-up ranged between $0-20 \%$, whereas the recurrence of syncope was always higher in untreated patients, who showed a rate of $20-60 \%$. In a meta-analysis of the three studies with a control group of untreated patients, ${ }^{36-38}$ syncope recurred in $9 \%$ of 85 patients treated with PM and in 38\% of 91 controls (relative risk: $0.24,95 \%$ confidence interval $(\mathrm{Cl})$ $0.12-0.48)$. In a large registry of 169 consecutive patients treated with $\mathrm{PM},{ }^{39}$ the actuarial estimate of syncopal recurrence was $7 \%$ at 1 year, $16 \%$ at 3 years and $20 \%$ at 5 years. Mixed form of CSS and pacing in VVI mode hampered the efficacy of pacing therapy. Finally, in a small study in which the diagnosis of a cardio-inhibitory carotid sinus syndrome was validated by ILR documentation of spontaneous asystolic pauses, ${ }^{40}$ syncope burden decreased from 1.68 (95\% Cl 1.66-1.70) episodes per patient per year before PM

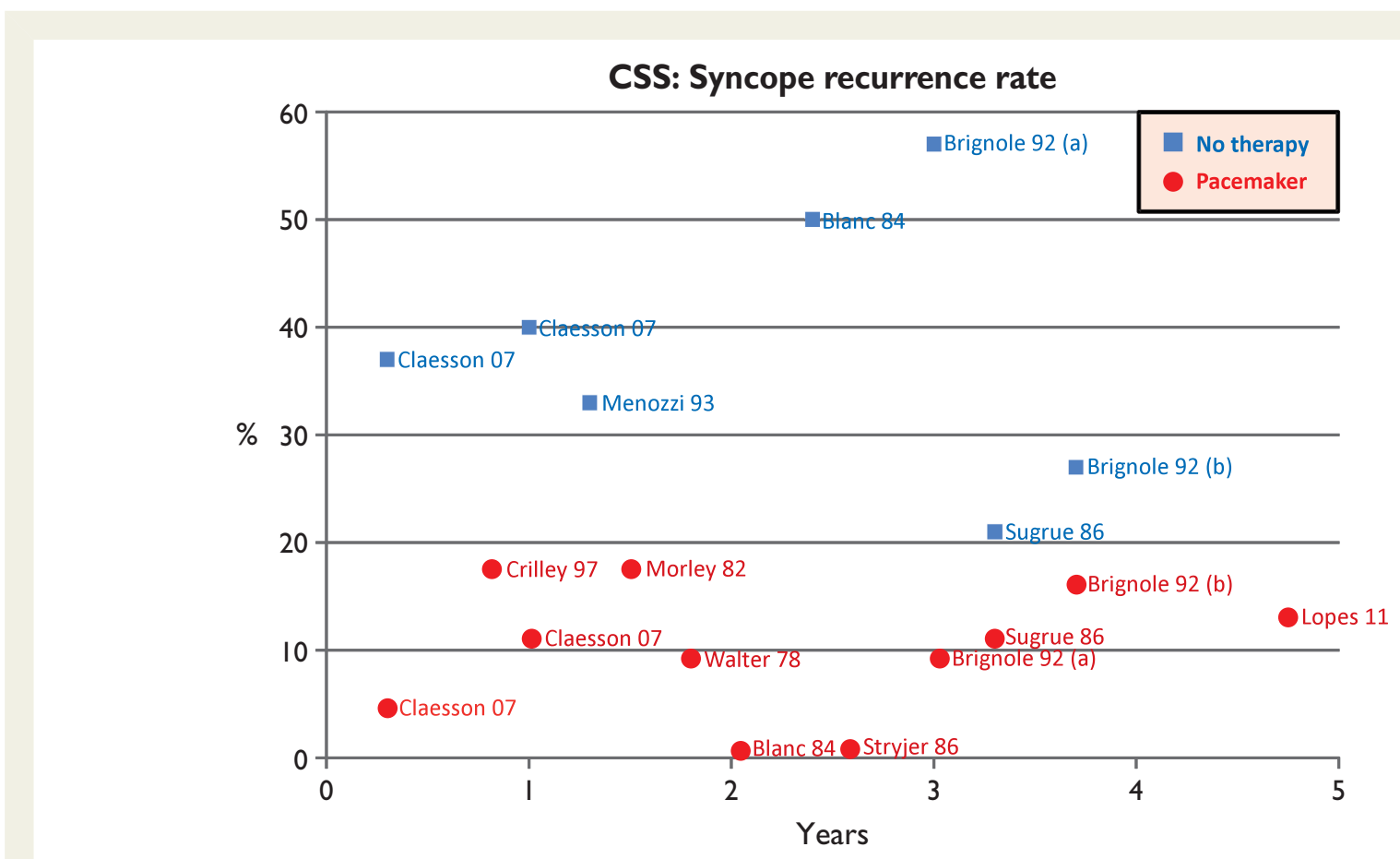

Figure 5 Recurrence of syncope in untreated and paced patients affected by carotid sinus syndrome (CSS). Reproduced with permission from Brignole et al. ${ }^{35}$ 
implantation to 0.04 (95\% Cl 0.038-0.042) after PM implantation ( $98 \%$ relative risk reduction). In conclusion, cardiac pacing is effective in preventing recurrences of syncope, but syncopal recurrence is expected to occur in up to $20 \%$ of patients within 5 years.

Despite the lack of large RCTs, the review of the literature supports the benefit of cardiac pacing in patients affected by carotid sinus syncope. A large randomized trial would be unlikely to change this knowledge.

In respect of the ESC Guidelines on Syncope, ${ }^{\mathrm{w} 25}$ the systematic literature review justifies a recommendation upgrade from class $2 a$ to class 1.

\section{Clinical perspectives:}

- The decision to implant a pacemaker should be made in the context of a relatively benign condition with the aim of preventing traumatic recurrences which are frequent in old patients with recurrent carotid sinus syncope.

- Since carotid sinus syndrome does not affect survival, the reduction of the burden of syncope (i.e. number of syncopes per patient peryear) and the reduction of related morbidities (i.e. trauma secondary to syncope) should probably better describe the benefit of cardiac pacing therapy.

- Compared with the natural history of carotid sinus syndrome, we can expect that the patients who receive a pacemaker will have about a $75 \%$ reduction of recurrences. However, syncopal recurrences are still expected to occur in up to $20 \%$ of paced patients within 5 years.

- Mixed forms of CSS and VVI mode hamper the efficacy of pacing therapy. In addition, cardiac pacing is not effective in preventing presyncopal recurrences.

\section{Choice of pacing mode (Recommendation 4)}

The optimal pacing mode is dual-chamber. In an acute intra-patient study, ${ }^{41} \mathrm{VVI}$ mode caused an important deterioration, compared with $D V I$ pacing characterized by a greater fall of systolic blood pressure ( 59 vs. $37 \mathrm{mmHg} ; P=0.001)$ and higher rate of symptom persistence ( 91 vs. $27 \% ; P=0.008$ ). In a 2 -month randomized crossover study on DVI vs. VVI mode, performed in 23 patients affected by mixed carotid sinus syndrome, ${ }^{42}$ syncope occurred in 0 vs. $13 \%(P=0.25)$, pre-syncope in 48 vs. $74 \%(P=0.04)$; DVI was the mode preferred by $64 \%$ of the patients, whereas the remaining $36 \%$ did not express any preference $(P=0.001)$. In the Westminster study of 202 patients, ${ }^{43}$ syncope recurred in $9 \%$ of DDD-paced patients while, in $\mathrm{VVI}$-paced patients, the rate of recurrence was twice as high (18\%).

\section{Tilt-induced vasovagal syncope}

Typically, the vasovagal reflex induced during tilt table testing is both hypotensive and bradycardic. The rationale for efficacy of cardiac pacing is that the cardio-inhibitory reflex is dominant, since there is no role of pacing in preventing vasodilatation and hypotension. The lack of reproducibility of tilt testing limits its utility as a means of assessing therapy. Moreover, the mechanism of tilt-induced syncope is frequently different from that of the spontaneous syncope recorded with the ILR. ${ }^{\text {w25,w76 }}$

\section{Tilt-induced vasovagal syncope (Recommendations 2 and 3)}

Effectiveness of pacing has been studied in five multi-centre RCTs, ${ }^{20-24}$ three non-blinded trials gave positive results and two blinded trials gave negative results. ${ }^{21-24}$ In the randomized, openlabel SYncope Dlagnosis and Treatment [SYDIT] and VASIS-PM trials, ${ }^{20,24}$ patients were selected on the basis of a positive cardio-inhibitory (mostly asystolic) response during tilt test. Syncopal recurrence at 2 years in the PM arm was 7\% in SYDIT and 6\% in VASIS-PM. Admittedly, any open-label trial has the potential for bias in reporting and assessment of outcomes. The Second Vasovagal Pacemaker Study (VPS II) and the Vasovagal SYNcope and PACing trial (SYNPACE) gave totally different results. ${ }^{22,23}$ The patients in these trials were younger (mean age: 50 years), had both cardio-inhibitory (mostly non-asystolic) and non-cardio-inhibitory responses during tilt test and patients in the control arm received a permanent PM programmed 'off'. Although there was a 30\% reduction in recurrence in the two groups $(95 \% \mathrm{Cl} 33$ to $63 \%$; $P=$ $0.14)$, the VPS II study failed to demonstrate significant superiority of pacing. In the SYNPACE trial, syncope recurred in $50 \%$ of patients assigned to pacing 'on' and in $38 \%$ of patients assigned to pacing 'off'. All these studies have limitations (particularly the pre-implantation selection criteria) and further studies are needed. Indeed, the ISSUE 2 study showed a weak correlation between the mechanism of syncope during tilt table testing and during spontaneous events documented by ILR. ${ }^{\text {w76 }}$ This finding suggests caution in implanting $\mathrm{PM}$, based on the tilt response. The clinical presentation is probably more important than tilt test positivity, in order to select patients who can benefit from cardiac pacing. In this regard, a high mean age, a history of recurrent syncope beginning in middle or older ages and frequent injuries, probably due to presentation without warning, characterized the ISSUE population (see section 3.2). ${ }^{\text {w74 }}$

Due to the contrasting results of the randomized trials, the estimate of benefit of pacing in cardio-inhibitory tilt-positive patients is weak. Divergence of opinion exists among experts. Further research is very likely to have an important impact on recommendations. Conversely, there is strong evidence that pacing cannot be offered to patients with non-cardio-inhibitory tilt-positive response and further tests (e.g. ILR) are warranted in order to document the mechanism of the spontaneous reflex.

\section{Clinical perspectives:}

- The fact that pacing may be effective does not mean that it is also always necessary. It must be emphasized that the decision to implant a pacemaker needs to be made in the clinical context of a benign condition that frequently affects young patients. Thus, cardiac pacing should be limited, as a last resort choice, to a highly selected small proportion of patients affected by severe reflex syncope. Patients suitable for cardiac pacing are probably those $>60$ years with a history of recurrent syncope beginning in middle or older ages and with frequent injuries, probably due to presentation without warning.

- Syncopal recurrences are still expected to occur despite cardiac pacing in a minority of patients.

- The weak correlation between the mechanism of syncope during tilt test and during spontaneous events suggests caution in implanting a pacemaker based on the tilt response.

\section{Choice of pacing mode (Recommendation 5)}

In all trials, ${ }^{20-24}$ dual-chamber pacing was used, with a rate drop response feature of the PM that instituted rapid DDD pacing if the device detected a rapid decrease in heart rate, but no comparison with conventional dual-chamber pacing has ever been made. 


\section{Indication for cardiac pacing in patients with undocumented reflex syncope}

\begin{tabular}{|l|c|c|c|}
\hline Recommendations & Class $^{\text {a }}$ & Level $^{\text {b }}$ & Ref. $^{\text {C }}$ \\
\hline $\begin{array}{l}\text { I) Carotid sinus } \\
\text { syncope. } \\
\text { Pacing is indicated in patients } \\
\text { with dominant cardioinhibitory } \\
\text { carotid sinus syndrome and } \\
\text { recurrent unpredictable } \\
\text { syncope. }\end{array}$ & & & \\
\hline $\begin{array}{l}\text { 2) Tilt-induced } \\
\text { cardioinhibitory } \\
\text { syncope. } \\
\text { Pacing may be indicated in } \\
\text { patients with tilt-induced } \\
\text { cardioinhibitory response } \\
\text { with recurrent frequent } \\
\text { unpredictable syncope and } \\
\text { age }>40 \text { years after alternative } \\
\text { therapy has failed. }\end{array}$ & B & \\
\hline $\begin{array}{l}\text { 3) Tilt-induced } \\
\text { non-cardioinhibitory } \\
\text { syncope. } \\
\text { Cardiac pacing is not } \\
\text { indicated in the absence of a } \\
\text { documented cardioinhibitory } \\
\text { reflex. }\end{array}$ & & \\
\hline
\end{tabular}

${ }^{a}$ Class of recommendation.

bevel of evidence.

${ }^{\mathrm{c}}$ Reference(s) supporting recommendation(s).

\section{Choice of pacing mode}

\begin{tabular}{|c|c|c|c|}
\hline Recommendations & Class $^{a}$ & Level $^{\text {b }}$ & Ref. ${ }^{c}$ \\
\hline $\begin{array}{l}\text { 4) Carotid sinus } \\
\text { syncope. } \\
\text { In patients with carotid sinus } \\
\text { syndrome, dual-chamber } \\
\text { pacing is the preferred mode } \\
\text { of pacing. }\end{array}$ & I & $\mathbf{B}$ & $41-43$ \\
\hline $\begin{array}{l}\text { 5) Tilt-induced } \\
\text { cardioinhibitory } \\
\text { syncope. } \\
\text { In patients with } \\
\text { cardioinhibitory vasovagal } \\
\text { syncope, dual-chamber pacing } \\
\text { is the preferred mode of } \\
\text { pacing. }\end{array}$ & I & C & - \\
\hline $\begin{array}{l}\text { 6) Lower rate and rate } \\
\text { hysteresis should be } \\
\text { programmed in order to } \\
\text { achieve back-up pacing } \\
\text { function which preserves } \\
\text { native heart rhythm and AV } \\
\text { conduction. }\end{array}$ & Ila & C & - \\
\hline
\end{tabular}

${ }^{\mathrm{a} C l a s s}$ of recommendation

${ }^{b}$ Level of evidence.

${ }^{\mathrm{c}}$ Reference(s) supporting recommendation(s).

\subsubsection{Unexplained syncope (and fall)}

The cause of syncope may remain unexplained at the end of a complete work-up performed in accordance with the recommendations of the 2009 ESC Guidelines for diagnosis and management of syncope. $^{\text {w25 }}$

\section{Unexplained syncope and positive adenosine triphosphate test (Recommendation 1)}

The role of the adenosine triphosphate test is controversial. Three studies showed no correlation between AV block induced by adenosine triphosphate and ECG findings (documented by ILR) during spontaneous syncope. ${ }^{w 76-w 78}$ Thus, the low predictive value of the test does not support its use as a solitary diagnostic test for selecting patients for cardiac pacing. Adenosine triphosphate may, however, have a role to play in assessing patients with unexplained, recurrent syncope, presenting without or with very short prodrome and absence of cardiac and ECG abnormalities, suspected to have an idiopathic paroxysmal AV block. ${ }^{4}$ In a small multi-centre trial performed on 80 highly selected elderly patients with unexplained syncope, who had a positive response to intravenous injection of a bolus of $20 \mathrm{mg}$ of adenosine triphosphate, dualchamber cardiac pacing significantly reduced 2-year syncopal recurrence from $69 \%$ in the control group to $23 \%$ in the active group. ${ }^{44}$ For the above considerations-whether the efficacy of pacing was due to adenosine triphosphate response or to other factors (i.e. patient selection) - remains to be determined. Because of this uncertainty over the mechanism of efficacy of pacing, this Task Force believes that the documentation of possible bradyarrhythmia in spontaneous syncope remains the preferred eligibility criterion for pacing.

\section{Unexplained syncope (Recommendation 2)}

In patients with unexplained syncope at the end of a complete work-up and absence of any conduction disturbance, the lack of a rationale and the negative results of a small controlled trial and of an observational trial give sufficient evidence of inefficacy of cardiac pacing. ${ }^{w 79, w 80}$ Thus, PM therapy is not recommended until a diagnosis is made.

\section{Unexplained falls (Recommendation 3)}

It has been estimated that $15-20 \%$ of unexplained falls may be syncopal in nature, possibly bradyarrhythmic; retrograde amnesia, which is frequent in the falling elderly, is responsible for misinterpretation of the event. However, in a randomized double-blind trial, ${ }^{45}$ cardiac pacing has been shown to be ineffective in preventing recurrences in patients with unexplained fall and carotid sinus hypersensitivity.

\section{Clinical perspectives:}

- In patients with recurrent, unexplained syncopes or falls at the end of the conventional work-up, ILR monitoring should be considered in an attempt to document a spontaneous relapse, instead of embarking on empiric cardiac pacing. 
Indication for cardiac pacing in patients with unexplained syncope

\begin{tabular}{|l|c|c|c|}
\hline Recommendations & Class $^{\text {a }}$ & Level $^{\text {b }}$ & Ref. $^{\text {c }}$ \\
\hline $\begin{array}{l}\text { I) Unexplained syncope } \\
\text { and positive adenosine } \\
\text { triphosphate test. } \\
\text { Pacing may be useful to reduce } \\
\text { syncopal recurrences. }\end{array}$ & IIb & B & 4,44 \\
\hline $\begin{array}{l}\text { 2) Unexplained syncope. } \\
\text { Pacing is not indicated in } \\
\text { patients with unexplained } \\
\text { syncope without evidence of } \\
\text { bradycardia or conduction } \\
\text { disturbance. }\end{array}$ & III & C & - \\
\hline $\begin{array}{l}\text { 3) Unexplained falls. } \\
\text { Pacing is not indicated in } \\
\text { patients with unexplained falls. }\end{array}$ & III & B & \\
\hline
\end{tabular}

${ }^{\mathrm{a}}$ Class of recommendation.

bLevel of evidence.

${ }^{\mathrm{c}}$ Reference(s) supporting recommendation(s).

\section{Indications for cardiac resynchronization therapy}

\subsection{Epidemiology, prognosis, and pathophysiology of heart failure suitable for cardiac resynchronization therapy}

Approximately $2 \%$ of the adult population in developed countries has HF; most patients will be aged $>70$ years and about half will have an LVEF $<50 \%$. ${ }^{\text {w1 }}$ About $1 \%$ of emergency hospital admissions amongst adults are primarily due to heart failure, which contributes to a further $4 \%$, although these may be underestimated due to issues with diagnosis and case definition. ${ }^{\text {} 81}$ In the EuroHeart Failure survey, $36 \%$ of those who had LV function assessed had an LVEF $\leq 35 \%$ and, of these, $41 \%$ had a QRS duration $\geq 120 \mathrm{~ms} ; 7 \%$ had RBBB, 34\% had LBBB or other intraventricular conduction delay (IVCD) and 17\% had QRS $\geq 150$ ms. $^{\text {w82 }}$ In the Italian Network on CHF (IN-CHF) registry, 1391 patients (25\%) had complete LBBB, 336 (6\%) had complete RBBB and 339 (6\%) had other forms of IVCD. ${ }^{\text {w83 }}$ The annual incidence of $L B B B$ is about $10 \%$ in ambulatory patients with left ventricular systolic dysfunction (LVSD) and chronic HF. ${ }^{\text {w84 }}$

Based on current guideline criteria, ${ }^{\text {w85 }}$ only a small proportion of patients with HF (perhaps 5-10\%) are indicated for CRT but this is still a large number of patients. Based on data from two EuroHeart Failure surveys and extrapolating from hospital discharge statistics, ${ }^{\text {w82,w86,w87 }}$ we estimate that about 400 patients per million population per year might be suitable for CRT, or up to 400,000 patients per year in ESC countries.

\section{Proportion of patients with heart failure currently treated with cardiac resynchronization therapy}

In 2011, the average CRT implantation rate in western and central Europe was 140 units per million population, ${ }^{\text {w2 }}$ of which 107 units were CRT-D and 33 were cardiac resynchronization therapy and pacemaker (CRT-P). A marked increase in CRT implantation rate was observed between 2005 and 2011 (Web Figure 6, left panel), which was consistent between countries, despite vastly different absolute rates (Web Figure 6, right panel), which approached zero in some eastern European and Middle-Eastern countries. ${ }^{\text {w88 }}$

\section{Mortality of heart failure}

The prognosis of HF is generally poor. Of patients admitted to hospital with $\mathrm{HF}$, the one-year mortality is about $20 \%$ in those aged $>75$ years and $>40 \%$ if aged $>75$ years, despite contemporary pharmacological therapy. ${ }^{\text {87,w89 }}$ High-quality information on the prognosis of outpatient populations with $\mathrm{HF}$ is harder to find. Patients in clinical trials tend to be younger and with fewer co-morbidities than in clinical practice and consequently have a better prognosis, with an annual mortality of $5-10 \%$ in recent trials, even though the trial protocols excluded

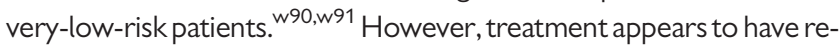
markably improved the prognosis of patients with chronic HF over the last 20 years. For example, the median life expectancy of patients enrolled in the Vasodilator in HEart Failure Trial V-HeFT-I trial (study period 1980-85), was just 3.5 years compared with more than 8 years for an age-equivalent population with moderately severe heart failure, treated with pharmacological therapy plus CRT, enrolled in CArdiac REsynchronization in Heart Failure (CARE-HF; study period 2001-2009). ${ }^{46, w 92, w 93}$ An ESC survey found that patients who received a CRT device had a one-year mortality of $<10 \% .{ }^{47}$

\section{Prognosis according to QRS morphology}

Patients with a broad QRS complex have a worse prognosis that may

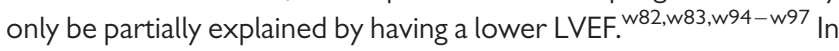
the ICD arm of the Multicenter Automatic Defibrillator Trial (MADIT) CRT study, the patients with IVCD, RBBB and LBBB had 3 -year mortality rates of 4,7 and $8 \%$, respectively. ${ }^{48}$

\section{Role of atrial fibrillation}

$\mathrm{AF}$ is the most common arrhythmia in patients with $\mathrm{HF}$. The Euro Heart Failure survey reported that up to $45 \%$ of patients with $\mathrm{HF}$ also presented with intermittent or permanent AF. ${ }^{\text {w82 }}$ The overall prevalence of new-onset $A F$ in patients hospitalized for congestive heart failure (CHF) was 13\%, ranging from $8-36 \%$ in different European regions. ${ }^{\text {w82,w86,w98 }}$ In chronic $\mathrm{HF}$, the prevalence of $\mathrm{AF}$ is linked directly to disease severity, ranging from $10-20 \%$ in mild-to-moderate CHF up to $50 \%$ in patients with advanced disease. ${ }^{\text {w99 }}$ Atrial fibrillation is a common cause of worsening HF and complicates management. Incident $A F$ is associated with a worse prognosis but it is unclear whether patients with chronic AF have a worse prognosis than those in sinus rhythm (SR), after correcting for age and co-morbidity. ${ }^{\text {w100- }}$

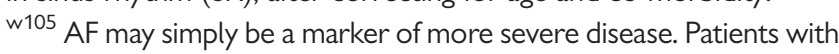
chronic AF are those who have tolerated and survived its development.

\section{Pathophysiology of heart failure relevant to cardiac resynchro- nization therapy}

Cardiac dyssynchrony is complex and multifaceted. Prolongation of the AV interval delays systolic contraction, which might then encroach on early diastolic filling. ${ }^{\text {w106 }}$ Atrial pressure falls as the atria relax. If ventricular contraction is delayed, then LV diastolic pressures will exceed atrial pressure causing diastolic mitral regurgitation. The loss of ventricular pre-load then leads to a reduction in LV 
contractility, due to loss of the Starling mechanism. Both inter- and intra-ventricular conduction delays lead to asynchronous contraction of LV wall regions (ventricular dyssynchrony), impairing cardiac efficiency and reducing stroke volume and systolic blood pressure. Poorly coordinated papillary muscle function may cause or aggravate functional systolic mitral regurgitation. Impaired performance promotes adverse LV remodelling.

Cardiac resynchronization therapy helps to restore $\mathrm{AV}$, inter- and intra-ventricular synchrony, improving LV function, reducing functional mitral regurgitation and inducing LV reverse remodelling, as evidenced by increases in LV filling time and LVEF, and decreases in LV end-diastolic- and end-systolic volumes, mitral regurgitation and septal dyskinesis. ${ }^{49,50, w 107}$ The dominant mechanism of benefit is likely to vary from one patient to the next and within an individual patient over time. It is possible that no single measure will accurately predict the response to CRT, since the mechanism of benefit is so heterogeneous. $^{\text {w108,w109 }}$

\section{Section 3.2 Patients in sinus rhythm}

\subsubsection{Indications for cardiac resynchronization therapy}

\subsubsection{Patients in New York Heart Association functional class III-IV}

There is conclusive evidence of both short- and long-term benefits of CRT in patients with New York Heart Association (NYHA) class III HF from a series of RCTs. The first randomized trials demonstrated the benefits of CRT on symptoms, exercise capacity and LV structure and function. ${ }^{49,51-54}$ The CARE-HF and COmparison of Medical Therapy, Pacing and Defibrillation in Heart Failure (COMPANION) trials evaluated the effects of CRT-P on $\mathrm{HF}$ hospitalizations and all-cause mortality. ${ }^{55,56} \mathrm{~A}$ recent meta-analysis showed that, in these patients, CRT improved symptoms and reduced all-cause mortality by $22 \%$ (risk ratio $0.78,95 \%$ $\mathrm{Cl} 0.67-0.91$ ) and $\mathrm{HF}$ hospitalizations by $35 \%$ (risk ratio $0.65,95 \%$ $\mathrm{Cl}$ 0.50-0.86). ${ }^{57}$ The evidence among NYHA class IV heart failure patients is limited, due to the low number of patients enrolled in RCTs (from 7 to 15\%). In a sub-study of the COMPANION trial, ${ }^{\text {w110 }}$ class IV patients who had had no scheduled or unscheduled HF hospitalizations during the last month (termed 'ambulatory' class IV) showed a significant reduction in the combined primary endpoint of time to all-cause mortality and hospitalization, but only a trend for all-cause mortality and HF deaths. Table 10 summarizes the main randomized CRT trials that included patients with NYHA class III-IV HF symptoms, sinus rhythm, poor LVEF $(\leq 35 \%)$ and prolonged QRS interval ( $\geq 120 \mathrm{~ms}$ ).

Duration of QRS interval $\geq 120 \mathrm{~ms}$ was the inclusion criterion used in most RCTs. Subgroup analysis, in a recent meta-analysis evaluating the impact of QRS duration on the efficacy of CRT, has shown that, in NYHA class III-IV HF patients, CRT significantly reduced all-cause mortality or hospitalization in patients with QRS duration $\geq 150 \mathrm{~ms}$ (data extracted from COMPANION and CARE-HF). ${ }^{58}$ The magnitude of effect and certainty of benefit declined with shorter QRS duration. Furthermore, most patients in the RCTs had LBBB morphology, which was associated with a more pronounced benefit, compared with non-LBBB patients (defined in the Web Table 11). ${ }^{48,59, w 111}$ The relationship between QRS duration and morphology requires further exploration.

\subsubsection{Patients in New York Heart Association functional class I-II}

Four RCTs have demonstrated that CRT improves LV function, allcause mortality and HF hospitalizations of patients with mild HF symptoms (NYHA class I-II), sinus rhythm, LVEF $\leq 30-40 \%$ and QRS duration $\geq 120-130 \mathrm{~ms}^{50,60-62}$ However, improvement in functional status or quality of life among patients randomized to CRT were modest and not robust. Most patients enrolled had NYHA class II HF symptoms; only 15\% in REsynchronization reVErses Remodelling in Systolic left vEntricular dysfunction (REVERSE) and 18\% in MADIT-CRT were in NYHA class I. ${ }^{50,61}$ CRT did not reduce all-cause mortality or HF events among NYHA class I patients. Therefore, the recommendation is restricted to patients in NYHA class II.

Pre-specified subgroup analyses of data collected in the MADIT-CRT, REVERSE and Resynchronization-Defibrillation for Ambulatory Heart Failure Trial (RAFT) trials demonstrated that patients with a QRS duration $\geq 150 \mathrm{~ms}$ benefited most from CRT. ${ }^{50,61,62}$ Meta-analyses using aggregate data from randomized trials showed that CRT was effective in reducing adverse clinical events in patients with baseline QRS duration $\geq 150 \mathrm{~ms}$ and suggested that CRT might not reduce events in patients with a QRS $<150$ ms. $^{58}$

Sub-group analyses based on QRS morphology in the MADIT-CRT, RAFT and REVERSE trials, ${ }^{48,50,62,63}$ and a meta-analysis of COMPANION, CARE-HF, MADIT-CRT and RAFT, ${ }^{64}$ suggested that patients with complete LBBB (defined in Web Table 11) showed a greater benefit on the composite of morbidity/mortality from CRT, compared with patients with non-specific IVCD or RBBB. Whether this is also true when applied to the effect on mortality is uncertain. Also, patients with LBBB had longer QRS duration, and therefore analyses by morphology may be confounded by QRS duration. In particular, the MADIT-CRT trial showed that, in patients with LBBB, CRT-D reduced by $53 \%$ the risk of death or HF hospitalization, compared with ICD alone, whereas non-LBBB patients did not derive clinical benefit from CRT therapy (statistically not significant $24 \%$ increased risk). ${ }^{48}$ With the exception of NYHA functional class I, all the pre-specified subgroups based on age, QRS duration $\geq 150 \mathrm{~ms}$, LV volumes and LVEF showed consistent results that indicated a clinical benefit of CRT-D compared with ICD-only therapy in all subgroups of LBBB patients (Web Figure 7). In the non-LBBB patients, there was no evidence of clinical benefit from CRT-D, regardless of the subgroup evaluated (Web Figure 7). Similar results were observed in the RAFT and REVERSE trials. ${ }^{62,63}$ Based on this evidence, current class I recommendations were restricted to patients with complete LBBB.

\section{Conclusions (Recommendations 1, 2, and 3).}

There is strong evidence that CRT reduces mortality and hospitalization, improves cardiac function and structure in symptomatic chronic HF patients with optimal medical treatment, severely depressed LVEF (i.e. $\leq 35 \%$ ) and complete LBBB. In these patients, CRT was superior either to optimal medical therapy or to ICD alone. In these patients, further research is very unlikely to change our confidence in the estimate of effect.

There is no evidence of a substantial heterogeneity of effect on morbidity or on mortality amongst patients with HF in NYHA classes II, III 
Table 10 Inclusion criteria, design, endpoints, and main findings of the randomized clinical trials evaluating cardiac resynchronization therapy in heart failure patients and sinus rhythm

\begin{tabular}{|c|c|c|c|c|c|c|c|c|}
\hline Trial (ref) & No. & Design & NYHA & LVEF & QRS & $\begin{array}{l}\text { Primary } \\
\text { endpoints }\end{array}$ & Secondary endpoints & Main Findings \\
\hline MUSTIC-SR ${ }^{52}$ & 58 & $\begin{array}{l}\text { Single-blinded, } \\
\text { crossover, } \\
\text { randomized CRT vs. } \\
\text { OMT, } 6 \text { months }\end{array}$ & III & $<35 \%$ & $\geq 150$ & 6MWD & $\begin{array}{l}\text { NYHA class, QoL, } \\
\text { peak VO LV volumes, } \\
\text { MR hospitalizations, } \\
\text { mortality }\end{array}$ & $\begin{array}{l}\text { CRT-P improved 6MWD, } \\
\text { NYHA class, QoL, peak VO, } \\
\text { reduced LV volumes and MR } \\
\text { and reduced hospitalizations }\end{array}$ \\
\hline PATH-CHF ${ }^{51}$ & 41 & $\begin{array}{l}\text { Single-blinded, } \\
\text { crossover, } \\
\text { randomized RV vs. } \\
\text { LV vs. BiV, } \\
\text { I2 months }\end{array}$ & III-IV & NA & $\geq 150$ & $\begin{array}{l}\text { Peak VO, } \\
\text { 6MWD }\end{array}$ & $\begin{array}{l}\text { NYHA class, QoL } \\
\text { hospitalizations }\end{array}$ & $\begin{array}{c}\text { CRT-P improved NYHA class, } \\
\text { QoL and 6MWD and reduced } \\
\text { hospitalizations }\end{array}$ \\
\hline $\mathrm{MIRACLE}^{49}$ & 453 & $\begin{array}{l}\text { Double-blinded, } \\
\text { randomized CRT vs. } \\
\text { OMT, } 6 \text { months }\end{array}$ & III-IV & $\leq 35 \%$ & $\geq 130$ & $\begin{array}{l}\text { NYHA class, } \\
\text { 6MWD, QoL }\end{array}$ & $\begin{array}{l}\text { Peak VO, LVEDD, } \\
\text { LVEF, MR } \\
\text { clinical composite } \\
\text { response }\end{array}$ & $\begin{array}{l}\text { CRT-P improved NYHA class, } \\
\text { QoL and 6MWD and reduced } \\
\text { LVEDD, MR and increased LVEF }\end{array}$ \\
\hline MIRACLE-ICD ${ }^{54}$ & 369 & $\begin{array}{l}\text { Double-blinded, } \\
\text { randomized } \\
\text { CRT-D vs. ICD, } \\
6 \text { months }\end{array}$ & III-IV & $\leq 35 \%$ & $\geq 130$ & $\begin{array}{l}\text { NYHA class, } \\
\text { 6MWD, QoL }\end{array}$ & $\begin{array}{c}\text { Peak VO } \\
\text { LVEDD, LVEF, MR } \\
\text { clinical composite } \\
\text { response }\end{array}$ & $\begin{array}{c}\text { CRT-D improved NYHA class, } \\
\text { QoL, peak } \mathrm{VO}_{2}\end{array}$ \\
\hline CONTAK-CD ${ }^{53}$ & 490 & $\begin{array}{l}\text { Double-blinded } \\
\text { randomized } \\
\text { CRT-D vs. ICD, } \\
6 \text { months }\end{array}$ & $\begin{array}{l}\text { II-III- } \\
\text { IV }\end{array}$ & $\leq 35 \%$ & $\geq 120$ & $\begin{array}{l}\text { NYHA class, } \\
\text { 6MWD, QoL }\end{array}$ & $\begin{array}{l}\text { LV volume, LVEF } \\
\text { composite of } \\
\text { mortality, VT/VF, } \\
\text { hospitalizations }\end{array}$ & $\begin{array}{l}\text { CRT-D improved 6MWD, } \\
\text { NYHA class, QoL, } \\
\text { reduced LV volume and } \\
\text { increased LVEF }\end{array}$ \\
\hline MIRACLE-ICD $\|^{60}$ & 186 & $\begin{array}{l}\text { Double-blinded, } \\
\text { randomized } \\
\text { CRT-D vs. ICD, } \\
6 \text { months }\end{array}$ & II & $\leq 35 \%$ & $\geq 130$ & Peak $\mathrm{VO}_{2}$ & $\begin{array}{l}\text { VE/VCO }, \text { NYHA, } \\
\text { QoL, 6MWD, LV } \\
\text { volumes and EF, } \\
\text { composite clinical } \\
\text { endpoint }\end{array}$ & $\begin{array}{c}\text { CRT-D improved NYHA, } \\
\text { VE/CO and reduced } \\
\text { LV volumes and improved LVEF }\end{array}$ \\
\hline COMPANION ${ }^{55}$ & 1520 & $\begin{array}{l}\text { Double-blinded } \\
\text { randomized } \\
\text { OMT vs. CRT-P / or } \\
\text { vs. CRT-D, } \\
\text { I5 months }\end{array}$ & III-IV & $\leq 35 \%$ & $\geq 120$ & $\begin{array}{l}\text { All-cause } \\
\text { mortality or } \\
\text { hospitalization }\end{array}$ & $\begin{array}{l}\text { All-cause mortality, } \\
\text { cardiac mortality }\end{array}$ & $\begin{array}{l}\text { CRT-P and CRT-D reduced } \\
\text { all-cause mortality or } \\
\text { hospitalization }\end{array}$ \\
\hline CARE-HF ${ }^{56}$ & 813 & $\begin{array}{l}\text { Double-blinded } \\
\text { randomized } \\
\text { OMT vs. CRT-P } \\
29.4 \text { months }\end{array}$ & III-IV & $\leq 35 \%$ & $\geq 120$ & $\begin{array}{l}\text { All-cause } \\
\text { mortality or } \\
\text { hospitalization }\end{array}$ & $\begin{array}{l}\text { All-cause mortality, } \\
\text { NYHA class, QoL }\end{array}$ & $\begin{array}{c}\text { CRT-P reduced all-cause } \\
\text { mortality and hospitalization } \\
\text { and improved NYHA class and } \\
\text { QoL }\end{array}$ \\
\hline REVERSE $^{61}$ & 610 & $\begin{array}{l}\text { Double-blinded, } \\
\text { randomized } \\
\text { CRT-ON vs. } \\
\text { CRT-OFF, } \\
\text { I2 months }\end{array}$ & I-II & $\leq 40 \%$ & $\geq 120$ & $\begin{array}{l}\% \text { worsened } \\
\text { by clinical } \\
\text { composite } \\
\text { endpoint }\end{array}$ & $\begin{array}{l}\text { LVESV index, } \\
\text { heart failure } \\
\text { hospitalizations and } \\
\text { all-cause mortality }\end{array}$ & $\begin{array}{l}\text { CRT-P/CRT-D did not change } \\
\text { the primary endpoint and did } \\
\text { not reduce all-cause mortality } \\
\text { but reduced LVESV index and } \\
\text { heart failure hospitalizations. }\end{array}$ \\
\hline MADIT-CRT ${ }^{50}$ & 1820 & $\begin{array}{l}\text { Single-blinded, } \\
\text { randomized } \\
\text { CRT-D vs. ICD, } \\
\text { I2 months }\end{array}$ & I-II & $\leq 30 \%$ & $\geq 130$ & $\begin{array}{c}\text { All-cause } \\
\text { mortality or } \\
\text { heart failure } \\
\text { hospitalizations }\end{array}$ & $\begin{array}{c}\text { All-cause mortality } \\
\text { and LVESV }\end{array}$ & $\begin{array}{l}\text { CRT-D reduced the endpoint } \\
\text { heart failure hospitalizations or } \\
\text { all-cause mortality and LVESV. } \\
\text { CRT-D did not reduced } \\
\text { all-cause mortality }\end{array}$ \\
\hline RAFT $^{62}$ & 1798 & $\begin{array}{l}\text { Double-blinded, } \\
\text { randomized CRT-D } \\
\text { vs. ICD } \\
40 \text { months }\end{array}$ & II-III & $\leq 30 \%$ & $\geq 120$ & $\begin{array}{c}\text { All-cause } \\
\text { mortality or } \\
\text { heart failure } \\
\text { hospitalizations }\end{array}$ & $\begin{array}{c}\text { All-cause mortality } \\
\text { and cardiovascular } \\
\text { death }\end{array}$ & $\begin{array}{l}\text { CRT-D reduced the endpoint } \\
\text { all-cause mortality or heart } \\
\text { failure hospitalizations. In } \\
\text { NYHA III, CRT-D only reduced } \\
\text { significantly all-cause mortality }\end{array}$ \\
\hline
\end{tabular}

CARE-HF = Cardiac Resynchronization-Heart Failure; CONTAK-CD = CONTAK-Cardiac Defibrillator; COMPANION = Comparison of Medical Therapy, Pacing and Defibrillation in Heart Failure; CRT-D = cardiac resynchronization therapy with defibrillator; CRT-P = cardiac resynchronization therapy pacemaker; $L V=$ left ventricular; LVEDD = left ventricular end-diastolic dimension; LVEF = left ventricular ejection fraction; LVESV = left ventricular end-systolic volume; MADIT-CRT = Multicenter Automatic Defibrillator Implantation Trial with Cardiac Resynchronization Therapy; MIRACLE = Multicenter InSync Randomized Clinical Evaluation; MIRACLE-ICD = Multicenter InSync Implantable Cardioverter Defibrillator trial; $\mathrm{MR}=$ mitral regurgitation; $\mathrm{MUSTIC}=$ Multisite Stimulation in Cardiomyopathies; No. = number of patients; $\mathrm{NYHA}=$ New York Heart Association; PATH-CHF = Pacing Therapies in Congestive Heart Failure trial; QoL = quality-of-life score; RAFT= Resynchronization-Defibrillation for Ambulatory Heart Failure Trial; $\mathrm{VE} / \mathrm{VCO}_{2}=$ minute ventilation/minute volume carbon dioxide production; $\mathrm{VF}=$ ventricular fibrillation; $\mathrm{VO}_{2}=$ volume of oxygen; $\mathrm{VT}=$ ventricular tachycardia; $6 \mathrm{MWD}=$ 6-min walk distance. 
or ambulatory IV. Therefore, this Task Force agrees to merge the recommendations of the 2012 ESC Guidelines on HF, ${ }^{\text {w81 }}$ and to provide one common set of recommendations for all patients with symptomatic HF irrespective to NYHA class (II-IV).

In patients who do not meet the above criteria, the evidence of efficacy is weak and further research is likely to have an important impact on our confidence in the estimate of effect, especially for those in NYHA class I and IV and those with non-LBBB morphology with QRS duration $<150 \mathrm{~ms}$.

Finally, there is no evidence of benefit in patients with HF and QRS $<120$ ms. In the Cardiac REsynchronization THerapy IN Patients with Heart Failure and Narrow QRS (RethinQ) trial, ${ }^{65}$ CRT did not improve peak oxygen consumption (primary endpoint) or quality of life in the subgroup of patients with QRS $<120 \mathrm{~ms}$ and evidence of echocardiographic dyssynchrony. The study was of too short a duration to observe effects on morbidity and mortality. The recent randomized, double-blind Evaluation of Resynchronization Therapy for Heart Failure in Patients with a QRS Duration Lower Than $120 \mathrm{~ms}$ (LESSER-EARTH) trial, ${ }^{66}$ which compared active vs. inactive CRT therapy, was prematurely interrupted due to futility and safety concerns after 85 patients were randomized. Indeed, CRT was associated with a significant reduction in the 6-minute walk distance and a non-significant trend towards an increase in heart failure-related hospitalizations.

\section{Key evidence supporting recommendations:}

- The relative magnitude of the benefits in patients with mild symptoms (NYHA class II), in terms of mortality, hospitalization, cardiac function and structure, is similar to those observed in patients with NYHA class III symptoms. Therefore, this Task Force agreed to give unique recommendations for all patients with symptomatic HFand severely depressed LVEF.

- The evidence for recommending CRT in patients with NYHA class I is inconclusive, due to the low number of patients enrolled in RCTs.

- The evidence for recommending CRT in patients with NYHA class IV is inconclusive, due to the low number of patients enrolled in RCTs. However, an individual situation should be taken into account, especially for NYHA class IV patients who have had no scheduled or unscheduled HF hospitalizations during the last month (termed 'ambulatory' class IV) in order to reduce hospitalizations and to improve symptoms.

- LBBB morphology (defined in the Web Table 11) is required in class I recommendation. Sub-analyses of randomized clinical trials and meta-analyses have shown that the beneficial effects of CRT were observed in patients with typical LBBB.

- Sub-analyses from randomized clinical trials suggest that the beneficial effects of CRT on morbidity and mortality and LV function may be greater in females, ${ }^{w 112}$ patients with non-ischaemic cardiomyopathy and patients with QRS duration $>150$ ms (the longer the QRS duration, the greater the benefit) (Figure 8). ${ }^{\text {w113 }}$

- The low number of HF patients with non-LBBB configuration included in randomized, controlled trials precludes firm conclusions for CRT implantation in this subgroup of patients. The evidence of benefit in patients with non-LBBB configuration is weak, particularly in patients with QRS $<150$ ms and NYHA classes I and II.

- RBBB most often implies a worse disease state than $\angle B B B$ and is generally expected not to benefit from CRT. For these patients, the decision to

\section{Magnitude of benefit from CRT}

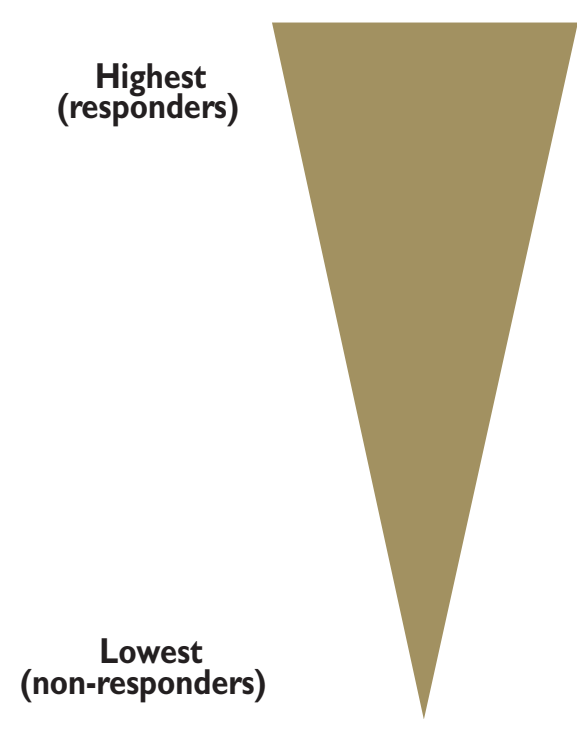

Wider QRS, left bundle branch block, females, non-ischaemic cardiomyopathy

Males, ischaemic cardiomyopathy

Narrower QRS, non-left bundle branch block

Figure 8 Clinical factors influencing the likelihood to respond to CRT. 
implant a CRT should be individualized, based on other clinical/imaging criteria.

- There is no evidence of benefit from CRT for patients with QRS $<120$ ms.

\section{Areas of dissent from recommendations:}

The recommendations given for indications for CRT (see below and Figure 8) represent a majority view of this Task Force but not all who contributed agreed. Several statements are based on subgroup analyses of RCTs that pose many problems with interpretation (interrelationship between QRS morphology and QRS duration, gender differences in response, prognostic benefit in ischaemic vs. non-ischaemic patients) or with areas of uncertainty that are still the objects of investigation (potential role of echocardiographic dyssynchrony in narrow QRS). Future studies might change our knowledge and recommendations.

3.2.1.3 Patient selection: role of imaging techniques to evaluate mechanical dyssynchrony criteria to select patients for cardiac resynchronization therapy

There is a spectrum of response to CRT, as with most other treatments. A substantial minority of patients have little or no symptomatic improvement after CRT and some will deteriorate, although this may reflect the natural history of the disease rather than the effect of CRT. Also, it is possible that the link between benefits to symptoms and prognosis may not be strong. Imaging techniques may be able to identify those patients who will respond favourably to CRT. ${ }^{\text {w114,w115 }}$ A sub-analysis of data collected in the CARE-HF trial showed that an interventricular mechanical delay (measured as the time difference between onset of pulmonary and aortic flow in pulsed wave Doppler spectral recordings) $\geq 49.2$ ms was an independent predictor of response to CRT. ${ }^{\text {w116 }}$ Many observational studies have demonstrated that the presence of LV dyssynchrony is associated with improved outcomes in patients treated with CRT. However, these results were challenged by the larger multi-centre and open PRedictors Of Response to Cardiac Resynchronization Therapy (PROSPECT) trial, where the tested echocardiographic parameters of cardiac mechanical dyssynchrony showed a modest accuracy to predict response to $\mathrm{CRT}$, defined by improvement in the composite clinical score and $\geq 15 \%$ reduction in LV end-systolic volume. ${ }^{w 117} A$ subsequent sub-study of this trial showed that the extent of LV reverse remodelling was associated with the extent of baseline interventricular and intra-left-ventricular mechanical dyssynchrony. ${ }^{\text {w118 }}$ After the PROSPECT trial, other imaging techniques were evaluated (magnetic resonance imaging, speckle tracking echocardiography, strain imaging, nuclear imaging) and yielded several parameters of LV mechanical dyssynchrony that have demonstrated to be independent determinants of CRT response and long-term outcome in several observational studies. ${ }^{\text {w119-w123 }}$ The real value of these novel technologies remains to be determined in randomized trials.

\section{Clinical perspectives:}

- Selection of HF patients for CRT based on LV mechanical dyssynchrony assessed with imaging techniques is uncertain and should therefore not be used as a selection criterion for CRT. However, data from several observational studies suggest that baseline LV mechanical dyssynchrony and acute resynchronization effect after CRT are independent determinants of CRT response and long-term outcome.

\section{Indications for cardiac resynchronization therapy in patients in sinus rhythm}

\begin{tabular}{|c|c|c|c|}
\hline Recommendations & Class $^{a}$ & Level $^{\mathrm{b}}$ & Ref. $^{c}$ \\
\hline $\begin{array}{l}\text { 1) LBBB with QRS } \\
\text { duration }>150 \text { ms. } \\
\text { CRT is recommended in } \\
\text { chronic HF patients and LVEF } \\
\leq 35 \% \text { who remain in NYHA } \\
\text { functional class II, III and } \\
\text { ambulatory IV despite adequate } \\
\text { medical treatment. }\end{array}$ & I & $\mathbf{A}$ & $48-64$ \\
\hline $\begin{array}{l}\text { 2) LBBB with QRS } \\
\text { duration I } 20-150 \text { ms. } \\
\text { CRT is recommended in } \\
\text { chronic HF patients and LVEF } \\
\leq 35 \% \text { who remain in NYHA } \\
\text { functional class II, III and } \\
\text { ambulatory IV despite adequate } \\
\text { medical treatment. }\end{array}$ & I & B & $48-64$ \\
\hline $\begin{array}{l}\text { 3) Non-LBBB with QRS } \\
\text { duration }>150 \text { ms. } \\
\text { CRT should be considered in } \\
\text { chronic HF patients and LVEF } \\
\leq 35 \% \text { who remain in NYHA } \\
\text { functional class II, III and } \\
\text { ambulatory IV despite adequate } \\
\text { medical treatment. }{ }^{d}\end{array}$ & Ila & B & $48-64$ \\
\hline $\begin{array}{l}\text { 4) Non-LBBB with QRS } \\
\text { duration I } 20-150 \text { ms. } \\
\text { CRT may be considered in } \\
\text { chronic HF patients and LVEF } \\
\leq 35 \% \text { who remain in NYHA } \\
\text { functional class II, III and } \\
\text { ambulatory IV despite adequate } \\
\text { medical treatment. }{ }^{d}\end{array}$ & Ilb & B & $48-64$ \\
\hline $\begin{array}{l}\text { 5) } C R T \text { in patients with } \\
\text { chronic } \mathrm{HF} \text { with QRS duration } \\
<120 \mathrm{~ms} \text { is not recommended. }\end{array}$ & III & B & 65,66 \\
\hline
\end{tabular}

$\mathrm{CRT}=$ cardiac resynchronization therapy; $\mathrm{HF}=$ heart failure; $\mathrm{LBBB}=$ left bundle branch block; LV = left ventricular; $L V E F=$ left ventricular ejection fraction; NYHA $=$ New York Heart Association.

${ }^{a}$ Class of recommendation.

bLevel of evidence.

'Reference(s) supporting recommendation(s).

'Patients should generally not be implanted during admission for acute decompensated HF. In such patients, guideline-indicated medical treatment should be optimized and the patient reviewed as an out-patient after stabilization. It is recognized that this may not always be possible.

\subsubsection{Choice of pacing mode (and cardiac resynchronization therapy optimization)}

The usual (standard) modality of CRT pacing consists of simultaneous ( $R V$ and LV) pacing with a sensed AV delay programmed between 100-120 ms with an LV lead possibly located in a lateral or posterolateral vein. This practice is largely empirically derived from pathophysiological reasoning and from the evidence provided by earlier clinical trials. ${ }^{55,56}$ Optimization of CRT has the objective of reducing the percentage of non-responders. In this respect, four major areas have been the subjects of research: 
- how to achieve biventricular pacing as close to $100 \%$ as possible;

- how to select the best LV lead position;

- how to program the AV interval in order to achieve the maximum contribution of LA contraction to LV filling (AV resynchronization); and

- how to eliminate the residual LV dyssynchrony after simultaneous biventricular pacing by selecting the timing of $R V$ and $L V$ pacing by means of device interventricular (VV) interval optimization (including, at its extreme, LV pacing alone).

For CRT optimization during follow-up, which goes beyond the scope of this guideline, we refer to a recent European and American expert consensus document. ${ }^{\text {w124 }}$

\section{Loss of biventricular pacing (Recommendation 1)}

Sustained and effective biventricular pacing is crucial to achieving the best results from CRT. In a recent trial involving $1812 \mathrm{HF}$ patients treated with CRT, a percentage of biventricular pacing between $93-100 \%$ was associated with a $44 \%$ reduction in the composite end point (all-cause mortality and heart failure hospitalization), compared with a percentage of biventricular pacing between 0-92\% (HR 0.56; $P=0.00001) .{ }^{67}$ These results were confirmed in 36935 patients, ${ }^{68}$ showing the greatest magnitude of reduction in mortality in patients with biventricular pacing exceeding $98 \%$ of all ventricular beats. Furthermore, in a cross-sectional analysis including 80768 patients, ${ }^{69}$ a percentage of biventricular pacing $>98 \%$ was achieved in only $59 \%$ of patients. For patients with $<98 \%$ biventricular pacing, the most frequent cause of pacing loss was inappropriately programmed long AV delay (accounting for $34 \%$ of cases) followed by atrial tachycardia/AF (31\% of cases) and premature ventricular complexes (17\% of cases). This evidence indicates that biventricular pacing has to be kept as close as possible to $100 \%$. How to achieve this goal goes beyond the scope of this guideline. We refer to a recent European and American expert consensus document. ${ }^{\text {w124 }}$

Selection of left ventricular lead position and single left ventricular vs. multiple site (Recommendations 2 and 3 )

The largest delay in mechanical contraction in an HF patient with LBBB is most often located in the LV posterolateral region, which is therefore also the preferred location to place the LV lead. A subanalysis of the COMPANION trial showed that anterior, lateral and posterior positions of the LV lead yielded similar clinical improvements and survival benefit. ${ }^{70}$ The REVERSE study indicated that a lateral LV lead position was associated with superior results concerning reverse LV remodelling and time to death and/or first HF hospitalization. $^{71}$ Data collected from the MADIT-CRT trial have demonstrated that basal or mid-ventricular positions of the LV lead portended superior long-term outcomes (defined as fewer hospitalizations for HF), compared with apical positions. ${ }^{72}$ These results may be explained by the presence of mid-ventricular or basal regions as the latest activated segments in patients with ventricular conduction disturbances. An apical position of the LV lead may be in close spatial relation with the $\mathrm{RV}$ lead, reducing the inter-electrode distance and precluding resynchronization. ${ }^{\text {w125-w127 }}$

However, several series have demonstrated that an LV lead position, coinciding with the regions of latest mechanical activation, yields superior outcomes compared with discordant positions. Individual variations in the conduction delay, which may modify the site of latest activation and the presence of transmural myocardial scar in the region targeted by the LV lead, may influence the results. ${ }^{\text {w128 }}$ The TARgeted Left Ventricular Lead Placement to Guide Cardiac Resynchronization Therapy (TARGET) trial randomized $220 \mathrm{HF}$ patients to an LV non-apical lead position, coincident with the latest activated areas (as assessed with speckle tracking echocardiography) or to standard unguided LV lead position. ${ }^{73}$ The group of patients with the LV lead positioned at the latest activated areas had a greater proportion of echocardiographic responders at 6 months follow-up (70 vs. $55 \% ; P=0.031)$, more clinical responders and lower rates of allcause mortality and HF hospitalizations (log-rank $P=0.0031$ ).

Regarding multiple-site LV pacing, a small study including 14 NYHA class III-IV HF patients in SR and LBBB demonstrated that dual-site LV pacing conferred larger acute haemodynamic improvements, compared with single-site LV pacing. ${ }^{\text {w129 }}$ Two small controlled trials showed some functional benefit. ${ }^{\mathrm{w} 130, w 131}$ Additional larger randomized trials with long-term clinical follow-up are needed in order to determine the real value of this pacing modality. Similarly, endocardial LV lead positioning has been shown to provide more homogeneous ventricular resynchronization and larger acute and mid-term improvements in LV function. ${ }^{\text {w132 }}$ However, the associated thrombo-embolic and infection complications need to be resolved before recommending this pacing modality. Ongoing randomized trials using wireless leads will provide further evidence to this field.

\section{Cardiac resynchronization therapy device optimization}

Observational studies have identified suboptimal programming of the $\mathrm{AV}$ and/or $\mathrm{VV}$ delays as determinant factors of poor response to CRT. $^{\text {w133 }}$ Small randomized trial and some observational series have shown significant improvements in HF symptoms and HF hospitalizations following optimization of the AV and $\mathrm{VV}$ delays, ${ }^{74,75, w 134-w 143}$ especially in ischaemic HF patients. ${ }^{\text {w144 }}$ These findings were not corroborated by the results of larger multicentre trials, ${ }^{76-83}$ which suggested that routine $\mathrm{AV}$ and $\mathrm{VV}$ delay optimization has limited effect on clinical or echocardiographic outcomes in CRT recipients, compared with a fixed 100-120 ms AV delay and simultaneous biventricular pacing (RV and LV) (Web Table 12). Patient selection, procedural timings and methodology employed (device algorithms, ECG or echocardiography) were not homogeneous across the studies, thus preventing firm conclusions from being obtained. Therefore, current evidence does not support AV and $\mathrm{V}$ optimization routinely in all patients receiving CRT.

However, in non-responders to CRT, in patients with ischaemic heart disease or in need of atrial pacing, evaluation of $\mathrm{AV}$ and $\mathrm{VV}$ delay may be recommended in order to correct suboptimal device settings (Web Figure 9). Several methods have been proposed to optimize the AV and VV delays (Table 13). ${ }^{74-83}$ These methods can be classified into two main groups: echocardiography-based and non-echocardiography-based methods. No clear difference between automatic electrocardiographic algorithms and echocardiographic CRT optimization has been found in The SMARTDelay Determined AV Optimization: a Comparison with Other AV Delay Methods Used in Cardiac Resynchronization Therapy 
Table I 3 Summary of current evidence for CRT optimization

\begin{tabular}{|c|c|c|c|c|}
\hline Parameter & $\begin{array}{l}\text { Standard } \\
\text { (current practice) }\end{array}$ & CRT optimization & $\begin{array}{l}\text { Additional clinical benefit } \\
\text { (compared to standard) }\end{array}$ & References \\
\hline LV lead position & Posterolateral & $\begin{array}{l}\text { - Avoid apical } \\
\text { - Target latest activated area }\end{array}$ & $\begin{array}{l}\text { Benefit likely (less hospitalization for HF) } \\
\text { Benefit likely (one RCT more responders, } \\
\text { less hospitalization for HF) }\end{array}$ & $\begin{array}{l}70-72 \\
73\end{array}$ \\
\hline AV delay & $\begin{array}{l}\text { Fixed empirical AV } \\
\text { interval } 120 \mathrm{~ms} \\
\text { (range } 100-120 \mathrm{~ms} \text { ) }\end{array}$ & $\begin{array}{l}\text { - Echo-Doppler: shortest AV delay without } \\
\text { truncation of the A-wave (Ritter's } \\
\text { method) or change in LV systolic function }\end{array}$ & $\begin{array}{l}\text { - Uncertain or mild (one small RCT and } \\
\text { several observational positive) }\end{array}$ & 74 \\
\hline & & $\begin{array}{l}\text { - Device-based algorithms } \\
\text { (SmartDelay, QuickOpt) }\end{array}$ & - Uncertain (two RCTs negative) & 76,79 \\
\hline VV delay & Simultaneous BiV & - Echo: residual LV dyssynchrony & $\begin{array}{l}\text { - Uncertain or mild (one RCT showed } \\
\text { mild benefit) }\end{array}$ & 77 \\
\hline & & - Echo-Doppler: largest stroke volume & $\begin{array}{l}\text { - Uncertain (one RCT negative, one } \\
\text { controlled positive) }\end{array}$ & 78,80 \\
\hline & & $\begin{array}{l}\text { - ECG: narrowest LV-paced QRS; difference } \\
\text { between BiV and preimplantation QRS }\end{array}$ & - Unknown (no comparative study) & 75 \\
\hline & & $\begin{array}{l}\text { Device-based algorithms (Expert-Ease, } \\
\text { Quick-Opt, Peak endocardial acceleration) }\end{array}$ & - Uncertain (three RCTs negative) & $76,82,83$ \\
\hline LV pacing alone & Simultaneous BiV & n.a. & Non-inferior & $84-88$ \\
\hline
\end{tabular}

$\mathrm{AV}=$ atrioventricular; $\mathrm{BiV}=$ biventricular; $\mathrm{CRT}=$ cardiac resynchronization therapy; $\mathrm{DTI}=$ tissue Doppler imaging; $\mathrm{HF}=$ heart failure; $\mathrm{LV}=$ left ventricular; n.a. = not available; $\mathrm{RCT}=$ randomized controlled trial; $\mathrm{W}=$ interventricular delay.

(SMART-AV), ${ }^{79}$ Optimization Study Using the QuickOpt Method (FREEDOM) $^{76}$ and Adaptive CRT trials. ${ }^{81}$

\section{Biventricular pacing vs. left ventricular pacing alone}

Biventricular pacing is the most common mode of delivering CRT. However, several studies have demonstrated the non-inferiority of LV pacing alone. The Bi vs. Left Ventricular Pacing: an International Pilot Evaluation on Heart Failure Patients with Ventricular Arrhythmias (BELIEVE) trial randomized, to biventricular- or LV pacing, 69 HF patients in NYHA functional class II-IV, QRS duration $\geq 130 \mathrm{~ms}$, LBBB and LVEF $\leq 35 \%$ who fulfilled the criteria for ICD implantation. ${ }^{84}$ After 12 months of follow-up, LV pacing induced similar improvements in clinical status, exercise capacity and LV dimensions and function, compared with biventricular pacing. These results were further confirmed in the recent Biventricular vs. LEFT Univentricular Pacing with ICD Back-up in Heart Failure Patients (B-LEFT) HF trial, which randomized 176 CRT-D recipients to biventricular or LV pacing. ${ }^{85}$ A meta-analysis of pooled data collected from 574 patients has shown that LV pacing induced similar improvements in 6-min walk distance, quality of life, NYHA functional class and peak oxygen consumption, compared with biventricular pacing. However, in terms of echocardiographic endpoints, biventricular pacing tended to induce larger improvements in LVEF and reductions in LV volumes, compared with LV pacing. ${ }^{86}$ The results of the multi-centre, double-blind, crossover Evaluation of Resynchronization Therapy for Heart Failure in Patients with a QRS Duration GREATER Than 120 ms (GREATEREARTH) trial showed that LV pacing was similar to biventricular pacing in terms of improvement in exercise capacity, LV function and volumes and $\mathrm{N}$-terminal pro-B type natriuretic peptide circulating levels. ${ }^{87}$ Moreover, a respective $21 \%$ and $17 \%$ of patients who did not respond clinically or echocardiographically to biventricular pacing, responded to LV pacing mode. Finally, a recent meta-analysis of five randomized trials for a total of 372 patients randomized to biventricular pacing and 258 to LV-only pacing showed that, in patients with moderate-to-severe HF, these two pacing modalities did not differ with regard to death/heart transplantation or need for hospitalizations. ${ }^{88}$

\section{Clinical perspectives:}

- The usual (standard) modality of CRT pacing consists of simultaneous biventricular pacing (RV and LV) with a fixed 100-120 ms AV delay with LV lead located in a posterolateral vein, if possible. This practice is largely empirical. However, AV and VV delay optimization has limited effect on clinical or echocardiographic outcomes in CRT recipients. Current evidence does not strongly support the performance of $A V$ and $\mathrm{V}$ optimization routinely in all patients receiving CRT.

- LV pacing alone, in non-pacemaker-dependent patients, seems to be non-inferior to biventricular pacing for improving soft endpoints (quality of life, exercise capacity and LV reverse remodelling) and might be considered, to lower the costs and complexity of the procedure and to increase the longevity of the device. LV pacing alone seems particularly appealing in children and young adults (see section 4.3).

- Additional trials are needed in order to determine the real value of multiple-site LV pacing. 


\section{Choice of pacing mode (and cardiac resynchronization therapy optimization)}

\begin{tabular}{|c|c|c|c|}
\hline Recommendations & Class $^{a}$ & Level $^{\mathrm{b}}$ & Ref. $^{c}$ \\
\hline $\begin{array}{l}\text { I) The goal of CRT should be } \\
\text { to achieve BiV pacing as close } \\
\text { to } 100 \% \text { as possible since the } \\
\text { survival benefit and reduction } \\
\text { in hospitalization are strongly } \\
\text { associated with an increasing } \\
\text { percentage of BiV pacing. }\end{array}$ & Ila & B & $67-69$ \\
\hline $\begin{array}{l}\text { 2) Apical position of the LV } \\
\text { lead should be avoided when } \\
\text { possible. }\end{array}$ & Ila & B & $70-72$ \\
\hline $\begin{array}{l}\text { 3) LV lead placement may be } \\
\text { targeted at the latest activated } \\
\text { LV segment. }\end{array}$ & Ilb & B & 73 \\
\hline $\begin{array}{l}\text { = cardiac resynchronization th } \\
\text { ss of recommendation. } \\
\text { el of evidence. } \\
\text { erence(s) supporting recommen }\end{array}$ & $\mathrm{n}(\mathrm{s})$ & & \\
\hline
\end{tabular}

\section{Section 3.3. Patients in atrial fibrillation}

Only patients with permanent AF or long-standing persistent AF will be considered in this document. For other forms of AF (i.e. paroxysmal or persistent) refer to the 2010 ESC Guidelines for the management of AF. ${ }^{\mathrm{w} 145}$ There are two ways of considering CRT for AF patients: (i) AF patients with moderate-to-severe HF with a haemodynamic indication for CRT and (ii) patients with a fast ventricular rate with HF or LV dysfunction justifying a strong rate control strategy with an $\mathrm{AV}$ junction ablation.

\subsubsection{Patients with heart failure, wide QRS, and reduced ejection fraction}

Despite a clear lack of evidence from randomized and controlled trials, in which only 200 patients with permanent AF have been included, ${ }^{89,90} 23 \%$ of patients who received CRT were in AF in a European clinical practice registry and an even higher number may be eligible. $^{\text {w146,w147 }}$ AF patients differ from SR patients because the ventricular rate is irregular and usually faster. The LV dysfunction in some AF patients may be a result of a tachycardiomyopathy process, in others poor LV function is the result of long-standing $\mathrm{HF}$; both situations are potentially correctable by rate control strategy using the AV junction ablation. ${ }^{\mathrm{w} 148-\mathrm{w} 150}$ Finally, AF patients in studies are generally older, sicker and have more co-morbidities and thus a worse prognosis, compared with SR patients, which may be reflected in the results comparing CRT effects in AF patients with the effects in SR patients. ${ }^{91,92}$ ICD back-up should be considered in patients at high risk of sudden death (see section 3.5).

\section{Heart failure, intrinsic QRS $\geq 120 \mathrm{~ms}$ and ejection fraction $\leq 35 \%$ (Recommendation $1 A$ and $1 B$ )}

The only prospective and randomized trial truly dedicated to patients with permanent AF and severe HF is the MUltisite STimulation In Cardiomyopathies (MUSTIC) AF trial. ${ }^{90,93}$ While the results were neutral in the intention-to-treat analysis, the per-protocol analysis including only patients with a biventricular pacing rate $>85 \%$ did show a slight but significant improvement in functional status at 6-month and 1-year follow-up. ${ }^{90,93}$ In the Ablate and Pace in Atrial Fibrillation (APAF) trial, ${ }^{89}$ the indication for CRT was primarily an indication for AV junction ablation; in the subgroups of patients with low LVEF, NYHA class $\geq$ III and QRS $\geq 120 \mathrm{~ms}$, CRT significantly reduced the primary endpoint, including death from HF, hospitalizations for $\mathrm{HF}$ or worsening of $\mathrm{HF}$, as well as a beneficial effect on $\mathrm{LV}$ reverse remodelling. The same findings were observed in the Left Ventricular-Based Cardiac Stimulation Post AV Nodal Ablation Evaluation (PAVE) trial. ${ }^{94}$ In order to overcome the lack of RCTs, some meta-analyses have compared the CRT results of patients with moderate or severe heart failure in SR with those in $A F^{91,92,95}$ In general, these studies showed that, in AF patients, the change in LVEF was similar-or even better-but the improvement in NYHA class, 6-min walk test or quality of life was significantly lower. Wilton et al., ${ }^{92}$ in a meta-analysis including 7495 patients from 33 observational studies with $22.5 \%$ AF patients, found a higher all-cause mortality in AF patients than in SR patients (10.8 vs. $7.1 \%$ per year; $P=$ 0.001). The risk of non-response to CRT was higher in AF patients (34.5 vs. $26.7 \% ; P=0.01$ ) and the presence of $A F$ was also associated with less improvement in quality of life scores, 6-min walk distance and LV end-systolic volume. In the RAFT trial, ${ }^{62}$ which included patients in NYHA class II, AF was present in $15 \%$ of the population; CRT seemed less efficient in AF patients than in SR patients at reducing the composite endpoint of death or hospitalizations for HF, but the interaction was not significant. In all these studies, the comparison was hampered by the difficulty of achieving an adequate biventricular pacing in AF patients.

Indeed, a major determinant of the success of CRT is the effective delivery of biventricular pacing. Data from large registries showed that a high percentage of biventricular pacing ( $\geq 99 \%)$ was a prerequisite for successful CRT pacing and that AF was a major determinant of loss of biventricular pacing (see also section 3.2.2). ${ }^{67-69}$ Failure of CRT was associated with new-onset AF during follow-up. ${ }^{\text {w151,w152 }}$ A particular aspect of AF patients is that AF rhythm with fast ventricular rate and irregularity may interfere with adequate biventricular pacing delivery. Competing AF rhythm - by creating spontaneous, fusion or pseudo-fusion beats-may reduce the rate of real biventricular capture. A careful analysis of the surface ECG is mandatory and in some cases a Holter recording could be useful, to assess the completeness of biventricular capture and to exclude pseudo-fusion, which the device algorithms might register as paced beats. ${ }^{96}$ In most AF patients with intact AV conduction, an adequate biventricular pacing delivery can be achieved only by means of AV junction ablation, and some authors require systematic $\mathrm{AV}$ junction ablation in these patients. It is important to emphasize that the use of AV junction ablation was highly variable between the different studies, ranging from $15 \%{ }^{97}$ to $100 \% .{ }^{90}$ The decision to perform AV junction ablation is still a matter of debate but most studies demonstrate a beneficial effect of enhancing the effects of CRT. ${ }^{98-102}$ In these studies, patients with AF without AV junction ablation had a worse response to CRT than patients in SR or AF with AV junction ablation. In a large, prospective, observational study, Gasparini et al. demonstrated that, over a long-term follow-up, the combination of CRT with AV junction ablation 
conferred improvements in LV function, functional capacity and exercise capacity (with the same magnitude as in patients with SR). ${ }^{101}$ In contrast, patients without completion of AV junction ablation had a worse result. In a recent systematic review, ${ }^{99} \mathrm{CRT}$ and $\mathrm{AV}$ junction ablation was associated with a substantial reduction in all-cause mortality (risk ratio 0.42 ) and cardiovascular mortality (risk ratio 0.44 ) and improvements in NYHA functional class (risk ratio 0.52), compared with CRT without AV junction ablation. Conversely, other studies, ${ }^{97,103-105}$ which showed similar improvement in SR and $A F$, support a conservative initial strategy in patients with AF by optimizing the medical treatment and programming the CRT device to obtain a higher percentage of ventricular pacing, and reserving $\mathrm{AV}$ node ablation only for patients with poor heart rate control.

In conclusion, the routine use of $\mathrm{AV}$ junction ablation ensures adequate biventricular pacing in patients with AF (Figure 10). The potential benefits must be balanced against the risks associated with creating PM dependency. AV junction ablation can be performed at the time of the CRT implantation or a few weeks later, after which time lead and device functioning are ensured.

Despite the weak evidence-due to lack of large randomized trials - the prevailing opinion of experts is in favour of the usefulness of CRT in AF patients with the same indications as for patients in $S R$, provided that AV junction ablation is added in those patients with incomplete $(<99 \%)$ biventricular capture. There are no data regarding NYHA class II patients.
These considerations justify upgrading the recommendation of the 2012 ESC Guidelines on HF from class Ilb to class Ila. ${ }^{\text {w81 Com- }}$ plete biventricular capture (either pharmacological or by means of AV junction ablation) is now warranted as a quality standard of practice.

Some experts were concerned about the paucity of evidence, from randomized trials, of benefit from CRT amongst patients with $\mathrm{AF}$, and felt that too much emphasis had been placed on the theory that the predominant mechanism of CRT is bi-ventricular stimulation. Much of the benefit of CRT could be due to atrioventricular-, rather than biventricular stimulation, in which case CRT may be less effective or ineffective in patients with AF since no atrioventricular stimulation can be provided in AF. Demonstrating that CRT is or is not effective in patients with AF should provide further insights into the mechanisms of CRT effect.

\subsubsection{Patients with uncontrolled heart rate who are candidates for atrioventricular junction ablation}

$\mathrm{AV}$ junction ablation and permanent pacing from the RV apex provides highly efficient rate control and regularization of the ventricular response in $A F$ and improves symptoms in selected patients. ${ }^{w 148-w 150, w 153-w 155}$ However, the downside is that LV dyssynchrony can be induced in about $50 \%$ of patients, ${ }^{\text {w1 } 156}$ which may be followed by worsening of HF symptoms. CRT may prevent the potential LV dyssynchrony induced by RV pacing and therefore

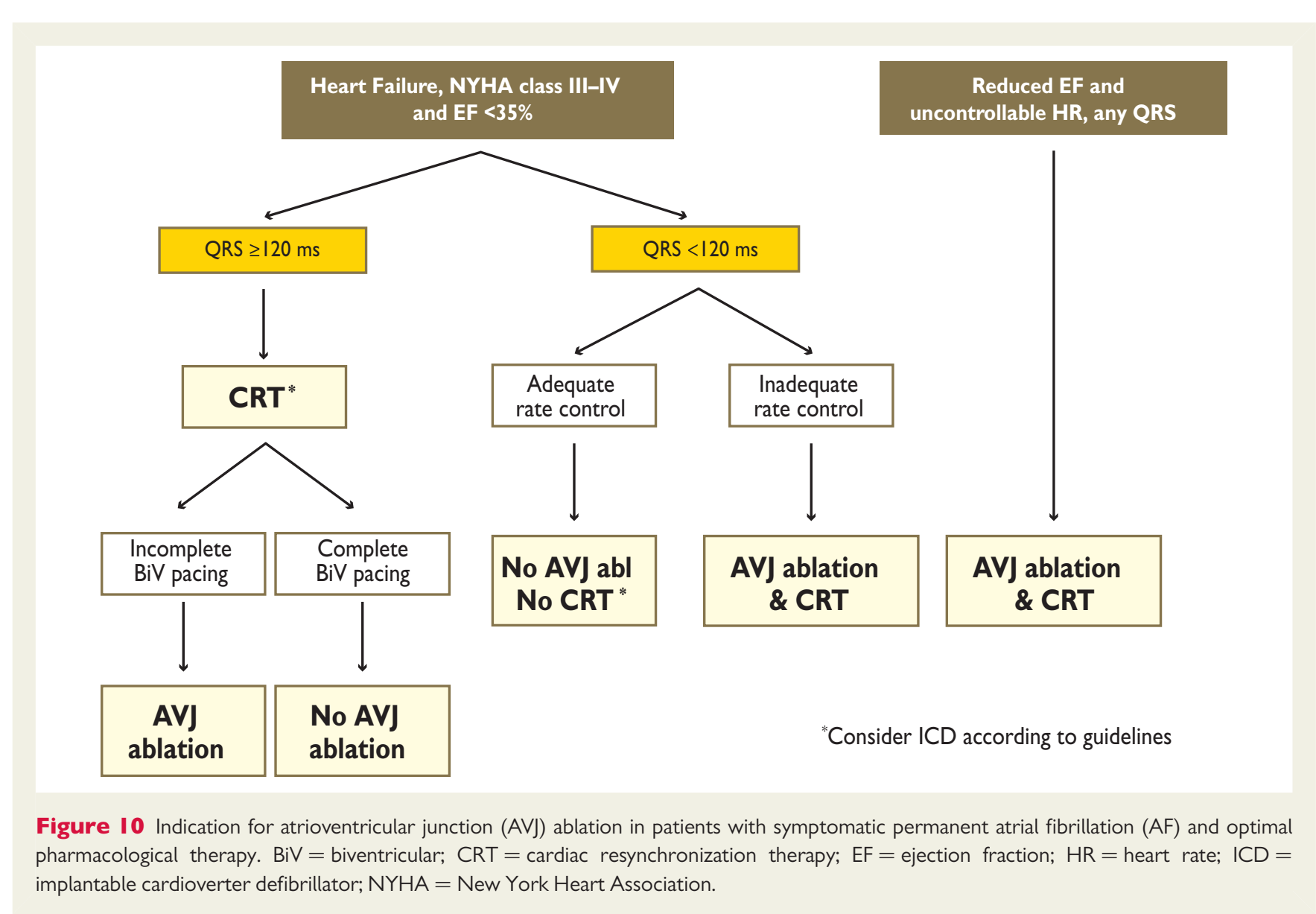


appears an interesting approach for patients eligible for $\mathrm{AV}$ junction ablation due to rapid $\mathrm{AF}$.

\section{Indications for cardiac resynchronization therapy (Recommendation 2)}

The PAVE, ${ }^{94}$ Optimal Pacing SITE (OPSITE) ${ }^{106}$ and AV Node Ablation with CLS and CRT Pacing Therapies for Treatment of AF (AVAIL CLS/CRT) ${ }^{105}$ trials showed that CRT exerted a modest but significant effect on quality of life, NYHA class and LVEF, as compared with $\mathrm{RV}$ pacing, in patients with $\mathrm{AF}$ and $\mathrm{AV}$ node ablation and various degrees of $L V$ function. The multi-centre, randomized and prospective APAF trial included 186 patients in whom a CRT or RV pacing device was implanted, followed by AV junction ablation. ${ }^{89}$ During a median follow-up of 20 months, CRT significantly decreased the primary composite endpoint (of death due to HF, hospitalization due to HF or worsening of HF) by $63 \%$ in the overall population. Compared with the RV pacing group, responders increased from 63 to $83 \%(P=0.003){ }^{107}$ The beneficial effects of CRT were similarly consistent in 46 patients (25\% of total) who had EF $\leq 35 \%$, NYHA class $\geq I I I$ and QRS width $\geq 120$, thus meeting the

\section{Indications for cardiac resynchronization therapy in patients with permanent atrial fibrillation}

\begin{tabular}{|c|c|c|c|}
\hline Recommendations & Class $^{a}$ & Level $^{\mathrm{b}}$ & Ref. $^{c}$ \\
\hline $\begin{array}{l}\text { I) Patients with HF, wide } \\
\text { QRS and reduced LVEF: }\end{array}$ & \multirow[b]{2}{*}{ Ila } & \multirow[b]{2}{*}{$\mathbf{B}$} & \multirow[b]{2}{*}{$62,89-95$} \\
\hline $\begin{array}{l}\text { IA) CRT should be } \\
\text { considered in chronic HF } \\
\text { patients, intrinsic } \mathrm{QRS} \geq \mathrm{I} 20 \\
\text { ms and LVEF } \leq 35 \% \text { who } \\
\text { remain in NYHA functional } \\
\text { class III and ambulatory IV } \\
\text { despite adequate medical } \\
\text { treatment }{ }^{\mathrm{d}} \text {, provided that a } \\
\text { BiV pacing as close to I00\% } \\
\text { as possible can be achieved. }\end{array}$ & & & \\
\hline $\begin{array}{l}\text { IB) AV junction ablation } \\
\text { should be added in case of } \\
\text { incomplete BiV pacing. }\end{array}$ & Ila & B & $\begin{array}{c}67-69,90 \\
96-105\end{array}$ \\
\hline $\begin{array}{l}\text { 2) Patients with } \\
\text { uncontrolled heart rate } \\
\text { who are candidates for } \\
\text { AV junction ablation. } \\
\text { CRT should be considered in } \\
\text { patients with reduced LVEF } \\
\text { who are candidates for AV } \\
\text { junction ablation for rate } \\
\text { control. }\end{array}$ & Illa & B & $\begin{array}{l}89,94 \\
105-107\end{array}$ \\
\hline
\end{tabular}

$\mathrm{AV}=$ atrioventricular; $\mathrm{CRT}=$ cardiac resynchronization therapy; $\mathrm{HF}=$ heart failure; ICD = implantable cardioverter defibrillator; LVEF = left ventricular ejection fraction; NYHA = New York Heart Association.

${ }^{a}$ Class of recommendation.

bLevel of evidence.

${ }^{\mathrm{c}}$ Reference(s) supporting recommendation(s).

${ }^{d}$ Patients should generally not be implanted during admission for acute

decompensated HF. In such patients, guideline-indicated medical treatment should be optimized and the patient reviewed as an out-patient after stabilization. It is recognized that this may not always be possible. requirements of the guidelines, to those observed in the other 140 patients $(75 \%$ of total) who did not $(\mathrm{HR}=0.32$ and 0.41 , respectively vs. RV pacing).

There is evidence, from small randomized trials, of an additional benefit of performing CRT pacing in patients with reduced EF, who are candidates for AV junction ablation for rate control, in order to reduce hospitalization and improve quality of life. However, the quality of evidence is moderate and discordance of opinion exists among experts. RCTs are warranted.

There is weak evidence that CRT is superior to RV pacing in patients with preserved systolic function.

Seen in perspective, there is a need for large RCTs to assess the efficacy of CRT in patients with permanent or longstanding, persistent AF, in terms of morbidity and mortality. These trials should compare not only CRT combined with medical treatment, but also CRT with AF ablation, which has been proposed and has to be evaluated as a potential treatment. ${ }^{\text {w157,w158 }}$

\subsection{Patients with heart failure and conventional pacemaker indications}

For patients with conventional PM who develop HF, upgrading from VVI or DDD to CRT devices represents an important part of the patient population implanted with a CRT device, being $23-28 \%$ of the CRT implantations in different registries. ${ }^{108, w 146}$ A de novo CRT indication in patients with conventional bradycardia indications is a matter of debate. Contrary to the recent 2012 ESC Guidelines on HF, ${ }^{\text {w81 }}$ separate recommendations are provided for these two situations.

\subsubsection{Patients with an indication for upgrading from conventional pacemaker or implantable cardioverter defibrillator to cardiac resynchronization therapy devices}

Previous studies have clearly shown that RV apical pacing might have deleterious effects on cardiac structure and function. ${ }^{\text {w156,w159 }}$ Moreover, different clinical trials have shown that there was a positive correlation between the rate of $\mathrm{R} V$ pacing and the occurrence of adverse events. ${ }^{17, w 160-w 162}$

\section{Upgrade from conventional pacemaker or implantable cardio- verter defibrillator (Recommendation 1)}

The additional benefit of biventricular pacing should be considered in patients requiring permanent or frequent $\mathrm{RV}$ pacing for bradycardia, who have symptomatic HF and low LVEF. Although large prospective, randomized trials specifically addressing the issue of upgrading to CRT are currently lacking, results from four small randomized trials are encouraging (Table 14). ${ }^{108-111}$ All of them had a crossover design, in which a 2-6 month period of CRT was compared with a 2-6 month period of RV pacing. The patients had conventional bradycardia indications (mostly permanent AV block), severe symptoms of HF (mostly NYHA class III or IV) and depressed EF (mostly <40\%). During the CRT study phase, the patients consistently showed clinical subjective improvement, less hospitalization and improved cardiac function, compared with the RV study phase.

The above results are consistent with those found in seven small observational studies, ${ }^{112-118}$ in which patients underwent CRT upgrade because of worsening of severe symptoms of HF and 
Table I4 Summary of evidence for upgrading from conventional pacemaker or implantable cardioverter defibrillator to cardiac resynchronization therapy devices

\begin{tabular}{|l|c|c|c|c|c|l|}
\hline Studies & No. of patients & $\begin{array}{c}\text { Echo, ESD } \\
(\%)\end{array}$ & $\begin{array}{c}\text { Echo, EF } \\
(\%)\end{array}$ & $\begin{array}{c}\text { QoL scores } \\
(\%)\end{array}$ & $\begin{array}{c}\text { NYHA class } \\
(\%)\end{array}$ & Clinical outcome \\
\hline RCT, cross-over design, upgraded CRT vs RV \\
\hline Hoijer ${ }^{\prime \prime}$ & 10 & -2 & - & Improved & - & Patient's preference: $90 \%$ CRT $(P=0.01)$ \\
\hline Leclercq $^{108}$ & 32 & -4 & 0 & -44 & -16 & $\begin{array}{l}\text { Fewer hospitalizations } \\
(4 \text { vs. I7, } P=0.00 I)\end{array}$ \\
\hline van Gerlop $^{\prime \prime \prime}$ & 36 & -9 & +18 & -10 & -16 & Responders, clinically relevant: $53 \%$ \\
\hline Delnoy & 309 & -31 & +30 & -19 & -26 & - \\
\hline Total & 118 & -6 & +17 & -22 & -18 & - \\
\hline
\end{tabular}

Observational studies, post-CRT upgrading vs. pre-CRT

\begin{tabular}{|c|c|c|c|c|c|c|}
\hline Leon 115 & 20 & -8 & +44 & -33 & -29 & Fewer hospitalizations: $-81 \%$ \\
\hline Baker ${ }^{112}$ & 60 & - & +26 & -31 & -29 & - \\
\hline Valls ${ }^{177}$ & 14 & -8 & +17 & - & -24 & - \\
\hline Eldadah ${ }^{1 / 3}$ & 12 & - & +16 & - & - & - \\
\hline Shimano 116 & 18 & - & +23 & - & -35 & Fewer hospitalizations: $-81 \%$ \\
\hline Laurenzi $^{114}$ & 38 & -5 & +41 & -68 & -36 & Responders, clinically relevant: $84 \%$ \\
\hline Vatankulu ${ }^{118}$ & 26 & -13 & +18 & - & - & - \\
\hline Total & 188 & -7 & +28 & -43 & -31 & \\
\hline
\end{tabular}

Controlled studies, upgraded CRT vs. de novo $\mathrm{CRT}^{\mathrm{a}}$

\begin{tabular}{|c|c|c|c|c|c|c|}
\hline Marai ${ }^{\mid 21}$ & 25 vs. 73 & -1 vs. -1 & +1 vs. +1 & - & -0.3 vs. -0.7 & $\begin{array}{l}\text { NYHA } \geq \text { I class: } 76 \text { vs. } 42 \% \\
(P=0.0 \mathrm{I})\end{array}$ \\
\hline Foley ${ }^{119}$ & 58 vs. 336 & - & +10 vs. +4 & Similar & Similar & $\begin{array}{l}\text { Responders: } 47 \text { vs. } 46 \% \\
\text { Mortality: } 27 \text { vs. } 26 \%\end{array}$ \\
\hline Paparella ${ }^{122}$ & 39 vs. 43 & - & +10 vs. +8 & - & -1.2 v.s -1.1 & $\begin{array}{l}\text { Hospitalization: }-8 \mathrm{I} \text { vs. }-77 \% \\
\text { Non-responders: } 9 \text { vs. } 10 \%\end{array}$ \\
\hline Frohlich ${ }^{120}$ & 70 vs. 102 & -7 vs. -6 & +10 vs. +10 & - & - & $\begin{array}{l}\text { NYHA } \geq 1 \text { class: } 53 \text { vs. } 51 \% \\
\text { Responders: } 56 \text { vs. } 56 \%\end{array}$ \\
\hline EU survey ${ }^{47}$ & 692 vs. 1675 & - & - & - & -1.0 vs. -1.0 & $\begin{array}{l}\text { At I-year follow-up: similar mortality ( } 8.6 \text { vs. } 7.9 \%) \text {, } \\
\text { hospitalization ( } 23 \text { vs. } 27 \% \text { ), improved quality of life } \\
\text { ( } 27 \text { vs. } 20 \% \text { ) and complications (II vs. } 10 \%)\end{array}$ \\
\hline Total & 884 vs. 2229 & - & - & - & - & \\
\hline
\end{tabular}

*Differences from baseline.

$\mathrm{CRT}=$ cardiac resynchronization therapy; ESD = end-systolic diameter; EF = ejection fraction; ICD = implantable cardioverter defibrillator; NYHA = New York Heart Association; $\mathrm{PM}=$ pacemaker; $\mathrm{QoL}=$ quality of life; $\mathrm{RCT}=$ randomized controlled trial; $\mathrm{RV}=$ right ventricle.

deterioration of cardiac function several months or years after RV pacing for bradycardia (Table 14). At the time of the upgrading procedure, almost all patients were in NYHA class III or IV and had EF $<35 \%$. In comparison with the period before CRT upgrade, these patients showed substantial subjective clinical improvement during the subsequent follow-up of 1-20 months, had fewer hospitalizations and demonstrated improved cardiac function.

Finally, five studies compared the clinical outcomes of patients who received an upgrade to CRT with those who received a de novo CRT implant for conventional indications. ${ }^{47,119-122}$ Baseline characteristics of the two groups were reasonably similar. During the subsequent follow-up of 3-38 months, upgraded patients showed improvement similar to the de novo patients (Table 14). In particular, the European CRT Survey, ${ }^{108}$ which compared 692 upgrades with 1675 de novo procedures at 141 centres in Europe, showed that there were no significant differences in clinical outcomes, mortality (Web Figure 11) or complication rates between upgrades and de novo procedures.

Upgrade to CRT is associated with a high complication rate, which was $18.7 \%$ in a recent large prospective trial. ${ }^{\text {w163 }}$ The decision to upgrade should therefore be made after careful assessment of the risk-benefit ratio (see also section 5, Complications).

Despite the lack of large randomized trials, there is sufficient evidence and general consensus that, in patients paced for conventional bradycardia indications who, during follow-up, develop severe symptoms of HF and have depressed EF, an upgrading to CRT pacing is likely to reduce hospitalization and improve their symptoms and cardiac performance. 
However, the quality of evidence is moderate and further research is likely to have an important impact on our confidence in the estimate of effect and might change the estimate. Moreover, the risk of complications is higher in upgrading procedures than in primary implantation procedures.

3.4.2. De novo cardiac resynchronization therapy pacing in patients with conventional indication for anti-bradycardia pacing (Recommendation 2)

Small randomized trials suggested that patients with moderate-to-severe LV dysfunction might benefit from CRT instead of conventional RV apical pacing (Table 15). ${ }^{123-130}$ In general, the primary endpoints of these trials were surrogate haemodynamic endpoints. While these trials consistently confirmed that chronic RV pacing leads to sustained and progressive deterioration of $L V$ function, and that this adverse remodelling process is prevented by CRT pacing, it is not yet known how this can translate into a better clinical outcome because of the lack of data on long-term clinical follow-up. Yu et al. compared RV apical pacing and CRT in a prospective, randomized double-blind study. ${ }^{130}$ Surprisingly, $50 \%$ of the study population had sinus node dysfunction, which is typically a condition with a contra-indication for permanent RV pacing. CRT prevented the reduction in LVEF and the increase in LV end-systolic volume observed at 1 year with RV apical pacing. No significant difference was observed in clinical endpoints for the two groups. The same findings were observed at 2-year follow-up. ${ }^{124}$ The PREventing VENTricular Dysfunction in Pacemaker Patients Without Advanced Heart Failure (PREVENT-HF) trial, ${ }^{129}$ randomly assigned 108 patients with high-degree AV block to CRT or RV pacing with or without an ICD, showed no advantage to CRT in terms of LV remodelling at 12 months. There were few clinical events with a trend in favour of CRT. In the Biventricular versus right ventricular pacing in patients with AV block (BLOCK HF) trial, ${ }^{125,126} 691$ patients with
AV block and systolic dysfunction were randomly assigned to CRT-P and RV pacing with or without an ICD and followed for an average of 37 months. The primary endpoint, a composite of mortality and urgent care visits for HF or a $15 \%$ or more increase in the LVSV, was significantly improved in those assigned to CRT. In a secondary analysis, hospitalization was $30 \%$ and mortality $17 \%$ lower in those assigned to CRT, although only the former achieved statistical significance. LV lead complications occurred in $6.5 \%$ of those assigned to CRT. The results of the ongoing Biventricular Pacing for Atrioventricular Block to Prevent Cardiac Desynchronization BIOPACE trial, ${ }^{\text {w164 }}$ which has a similar design, are awaited.

There is emerging evidence that de novo CRT implantation may reduce HF hospitalization, improve quality of life and reduce symptoms of HF in patients with history of HF, depressed cardiac function and a bradycardia indication for pacing. The benefit should be weighed against the added complication rate and costs of CRT devices and their shorter service life. The quality of evidence is low and further research is likely to have an important impact on our confidence in the estimate of effect, and may change the estimate.

\section{Clinical perspectives:}

- Owing to the heterogeneity of published studies, it is difficult to identify the brady-paced population who may benefit from upgrading to CRT. In general, however, it seems that patients who might benefit are those who-early or late after conventional permanent RV pacing - have a deterioration of $L V$ function (e.g. significant reduction of $E F$ to $<35 \%$ ), relevant worsening of symptoms of HF and hospitalizations for HF, despite optimal medical therapy. Owing to the lack of high-quality evidence, the indication for CRT remains largely individual.

- Late upgrade after HF development seems to provide similar benefit to de novo implantation in patients with initial preserved cardiac function. Therefore, a strategy of initially conventional anti-brady pacing, with later upgrade in case of worsening symptoms, seems reasonable.

Table I 5 Summary of evidence of RCTs of de novo CRT implantation compared with RV apical pacing in patients with conventional indication for anti-bradycardia pacing

\begin{tabular}{|c|c|c|c|c|c|c|}
\hline Studies & No. of patients & $\begin{array}{c}\text { Echo, ESV } \\
(\%)\end{array}$ & $\begin{array}{c}\text { Echo, EF } \\
(\%)\end{array}$ & $\begin{array}{c}\text { QoL scores } \\
\text { (\%) }\end{array}$ & $\begin{array}{c}\text { NYHA class } \\
(\%)\end{array}$ & Clinical outcome \\
\hline \multicolumn{7}{|c|}{ Patients with moderate/severe systolic dysfunction, CRT vs RV } \\
\hline HOBIPACE ${ }^{127}$ & 30 & -9 & +22 & -19 & -24 & Patient's preference: $67 \%$ CRT, $7 \%$ RV $(P=0.0002)$ \\
\hline COMBAT 128 & 60 & -24 & -21 & -47 & -24 & Worsening HF or hospitalization: 3 vs. 8 patients \\
\hline BLOCK HF ${ }^{125,} 126$ & 691 & - & - & - & - & $\begin{array}{l}\text { Significant } 28 \% \text { reduction in the combined primary endpoint of } \\
\text { mortality, heart-failure related urgent care, and increase in LV } \\
\text { end-systolic volume }\end{array}$ \\
\hline \multicolumn{7}{|c|}{ Patients with preserved systolic function, CRT vs RV } \\
\hline Albertsen 123 & 50 & - & +5 & - & -17 & - \\
\hline $\mathrm{PACE}^{124,130}$ & 177 & -22 & +13 & No difference & - & Hospitalization for HF: 6 vs. $7 \%$ (ns) \\
\hline PREVENT-HF 129 & 108 & -5 & +7 & - & - & Worsening of HF: 6 vs. $14 \%$ (ns) \\
\hline
\end{tabular}

$\mathrm{CRT}=$ cardiac resynchronization therapy; ESV = end-systolic volume; EF = ejection fraction; ICD = implantable cardioverter defibrillator; NYHA = New York Heart Association $\mathrm{PM}=$ pacemaker; $\mathrm{QoL}=$ quality of life; $\mathrm{RCT}=$ randomized controlled trial; $\mathrm{RV}=$ right ventricular; $\mathrm{ns}=$ not significant. 
- In patients being considered for de novo implantation, it is important to distinguish to what extent clinical presentation may be secondary to the underlying bradyarrhythmias, rather than to LV dysfunction. This is often difficult to recognize.

- In the decision-making process between upgraded and de novo CRT pacing instead of conventional RV pacing, physicians should take into account the added complication rate related to the more complex biventricular system, the shorter service life of CRT devices with the consequent need for earlier pacemaker replacement and the additional costs. See also section 5, Complications.

Indication for upgraded or de novo cardiac resynchronization therapy in patients with conventional pacemaker indications and heart failure

\begin{tabular}{|l|c|c|c|}
\hline Recommendations & Class $^{\mathrm{a}}$ & Level $^{\mathrm{b}}$ & Ref. $^{\mathrm{C}}$ \\
\hline I) Upgrade from & & \\
conventional PM or ICD. \\
CRT is indicated in HF patients \\
with LVEF <35\% and high \\
percentage of ventricular \\
pacing who remain in NYHA \\
class III and ambulatory IV \\
despite adequate medical \\
treatment.
\end{tabular}

$\mathrm{CRT}=$ cardiac resynchronization therapy; $\mathrm{HF}=$ heart failure; $I C D=$ implantable cardioverter defibrillator; LVEF = left ventricular ejection fraction; PM = pacemaker; NYHA = New York Heart Association.

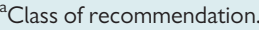

bLevel of evidence.

'Reference(s) supporting recommendation(s)

dPatients should generally not be implanted during admission for acute decompensated HF. In such patients, guideline-indicated medical treatment should be optimized and the patient reviewed as an out-patient after stabilization. It is recognized that this may not always be possible.

\section{Section 3.5 Back-up implantable cardioverter defibrillator in patients indicated for cardiac resynchronization therapy}

3.5.1 Benefit of adding cardiac resynchronization therapy in patients with indications for implantable cardioverter defibrillator (Recommendation 1)

Five large randomized trials compared the effects of CRT-D with ICD alone and showed an advantage for CRT-D in terms of survival, morbidity and symptom reduction. ${ }^{50,53,54,60,62}$ Therefore, when an ICD is indicated in secondary or primary prevention of sudden death, according to current guidelines to which we refer, ${ }^{\text {w69 }}$ it is recommended that CRT be added to improve symptoms, exercise tolerance and cardiac function and to reduce hospitalization in symptomatic chronic HF patients with optimal medical treatment, $\mathrm{LVEF} \leq 35 \%$ and complete LBBB, according to the recommendation reported in the section 3.2 and 3.3 of these Guidelines.

\subsubsection{Benefit of adding implantable cardioverter defibrillator in patients with indications for cardiac resynchronization therapy (Recommendation 2)}

Even though the theoretical reason for adding an ICD to CRT is clear - to reduce of the risk of arrhythmic death - the survival benefit of CRT-D over CRT-P is still a matter of debate, mainly because no RCT has been designed to compare these treatments.

COMPANION had three study arms - optimal medical therapy, CRT-P and CRT-D_but was not designed to compare CRT-D with CRT-P. ${ }^{55}$ Only CRT-D was associated with a significant decrease in total mortality at 1 year, compared with optimal medical therapy $(P=0.003)$, whereas the $24 \%$ relative risk reduction in the CRT-P arm was only marginally significant $(P=0.059)$. Sudden cardiac death was only significantly reduced by CRT-D, compared with medical therapy, over 16 months follow-up. Studies that compared CRT alone against optimal medical therapy overall, did not show a reduction in sudden cardiac death risk with $\mathrm{CRT}^{52,56}$ CARE-HF, with a 29-month follow-up period, was the first study to show a reduction in total mortality by CRT, compared with control, but without significant lowering of the risk of sudden cardiac death. ${ }^{56}$ However, in the extension study with a 37.4-month follow-up time, ${ }^{46}$ there was a significant $5.6 \%$ reduction in the absolute risk of dying suddenly. The results imply that, although the risk of dying from HF is immediately lowered by CRT, the reduction of risk of sudden cardiac death evolves at a much slower rate. It is very probable that reduction in risk of sudden cardiac death by CRT is related to the extent of reverse remodelling.

In a recent meta-analysis, ${ }^{57}$ which encompassed virtually all published trials on CRT, the mortality benefit of CRT was largely driven by a reduction in HF-related mortality. However, the CRT and control groups did not differ in their risk for sudden cardiac death (12 trials, 175 events in 3592 patients; RR 1.04; 95\% Cl 0.771.41). Another meta-analysis, ${ }^{131}$ made using a Bayesian approach, which included 12 studies (but not REVERSE, MADIT-CRT or RAFT) and encompassed 8307 patients and 1636 events, failed to show a superiority of CRT-D over CRT-P. The Bayesian approach models the multivariate intervention effects of multi-group trials and thus provides higher methodological quality than previous meta-analyses. Combined CRT and ICD therapy reduced the number of deaths by one third, compared with medical therapy alone [OR 0.57; 95\% Cl 0.40-0.80], but did not further improve survival when compared with ICD (OR $0.82 ; 95 \% \mathrm{Cl} 0.57$ to 1.18 ) or CRT (OR 0.85; 95\% Cl 0.60-1.22) therapy alone. In conclusion, the evidence from RCTs is insufficient to show the superiority of combined CRT and ICD over CRT alone. Nevertheless the Bayesian analysis, based on an extrapolated analysis, suggests that it is probable 
Table 16 Probability of best treatment for patients with left ventricular dysfunction (from a meta-analysis of 12 RCTs) 131

\begin{tabular}{|c|c|c|c|c|}
\hline \multirow{2}{*}{ Therapy } & \multicolumn{2}{|c|}{ All studies } & \multicolumn{2}{c|}{ NYHA III or IV HF } \\
\cline { 2 - 5 } & Overall mortality (\%) & Probability of best treatment & Overall mortality (\%) & Probability of best treatment \\
\hline Medical & 14.0 & 0 & 13.7 & 0 \\
\hline CRT & 10.3 & 0.14 & 10.5 & 0.27 \\
\hline ICD & 10.6 & 0.10 & 12.2 & 0.08 \\
\hline CRT + ICD & 9.1 & 0.75 & 9.7 & 0.62 \\
\hline
\end{tabular}

$\mathrm{CRT}=$ cardiac resynchronization therapy; $\mathrm{HF}=$ heart failure; ICD $=$ implantable cardioverter defibrillator; NYHA = New York Heart Association; RCTs = randomized controlled trials.

that combined therapy is the best option (probability of $75 \%$ in analysis) (Table 16).

\section{Selection of cardiac resynchronization therapy and defibrillator or cardiac resynchronization therapy and pacemaker}

There are reasons for preferentially implanting CRT-Ds in asymptomatic or mildly symptomatic patients. NYHA I-II patients are younger, have fewer co-morbidities and have a higher proportion of sudden- vs. non-sudden cardiac deaths. In Sudden Cardiac Death in Heart Failure Trial [SCD HeFT, ${ }^{w 165}$ a subgroup analysis revealed a greater benefit of ICD in NYHA II than in NYHA III patients. A significant $46 \%$ relative risk reduction of total mortality for the primary preventive ICD group was confined to NYHA II (representing $70 \%$ of patients) and could not be demonstrated in NYHA III. The possible survival benefit conferred by CRT-D in NYHA I-II must be balanced against the risk of ICD-related complications, in particular lead failure and inappropriate shocks. ${ }^{\mathrm{w} 166, \mathrm{w} 167}$ For patients in end-stage HF the individual patient situation must be taken into account when considering a CRT-D. In the COMPANION study, 217 out of 1520 patients were in NYHA class IV at baseline. ${ }^{\text {w110 }}$ Both CRT-D and CRT-P improved the primary endpoint of time to total mortality and allcause hospitalizations. In the medical therapy arm, CRT-P and CRT-D groups, 25, 16 and 9\%, respectively, died of sudden cardiac death over 2 years. Time to sudden cardiac death was only prolonged by CRT-D $(P=0.039)$ but did not differ significantly between the CRT-P and CRT-D treatment arms ( $P=0.07)$. Thus CRT-D is beneficial in all disease states, but the benefit appears relatively small in end-stage HF, in which the main reason for choice of device is related to improvement of quality of life and reduction of HF-related hospitalizations and death.

In the MADIT-II trial, risk stratification for primary preventive ICDs in ischaemic patients indicated five clinical factors predictive of total mortality in the control group and thus a potential for reduced benefit from ICD. These were NYHA class $\geq 11$, age $\geq 70$ years, blood urea nitrogen $\geq 26 \mathrm{mg} / \mathrm{dl}, \mathrm{QRS} \geq 120 \mathrm{~ms}$ and AF. ${ }^{\text {w168 }}{ }^{16 i g h t-}$ year benefit of ICD has been recently reported. ${ }^{\text {w169 }}$ Patients with low- (0 risk factors) and intermediate risk (1-2 risk factors) demonstrated a significantly higher probability of survival at 8-year follow-up when treated by ICD, compared with non-ICD therapy (75 vs. $58 \%$; $P$ $=0.004$ and 47 vs. $31 \% ; P=0.001$, respectively). By contrast, among high-risk patients ( 3 or more risk factors), there was no significant difference in 8-year survival between the ICD and non-ICD subgroups (19 vs. $17 \%)$

The cost-effectiveness of CRT-P or CRT-D in comparison with optimized medical therapy has been evaluated in several studies, taking into consideration different time horizons and using different modelling assumptions/contexts. ${ }^{\text {w170 }}$ Using a lifetime horizon and compared with optimal medical therapy, CRT-D is still a costeffective therapy below the benchmark of $\$ 50000$ per quality-adjusted life year (QALY) in USA. ${ }^{\text {w170 }}$ Thorough costeffectiveness analyses were performed in the COMPANION and CARE-HF trials. $^{\text {w171,w172 }}$ In COMPANION, intention-to-treat data were modelled to estimate the cost-effectiveness of CRT-D and CRT-P, compared with optimal medical treatment, over a base-case 7-year treatment episode. During 2 years of follow-up, hospitalization costs were reduced by 29 and 37\% for CRT-D and CRT-P, respectively. Based on a 7-year base-case time period analysis, the incremental cost-effectiveness ratios per life year gained were $\$ 46700$ and $\$ 28100$ for CRT-D and CRT-P, respectively. In addition, the incremental costs per QALY gained for CRT-D and CRT-P were $\$ 43000$ and $\$ 19600$, respectively. In the CARE-HF trial, during a mean follow-up of 29.4 months, the costeffectiveness analyses demonstrated that, compared with medical therapy, CRT-P was associated with an incremental costeffectiveness ratio of $€ 19319$ per QALY gained and $€ 43596$ per life-year gained. The REVERSE trial provides data on the costeffectiveness of CRT in patients with NYHA functional class I-II HF symptoms. ${ }^{\text {w173 }}$ Based on data from the 262 patients in the European cohort of this trial, the analysis demonstrated that CRT is a cost-effective therapy in patients with mild HF symptoms, showing an incremental cost-effectiveness ratio of $€ 14278$ per QALY gained.

\section{Clinical perspectives:}

- The evidence from RCTs is insufficient to show the superiority of combined CRT and ICD over CRT alone. Owing to the potential incremental survival benefit of CRT-D over CRT-P, the prevailing opinion among the members of this Task Force is in favour of a superiority of CRT-D in terms of total mortality and sudden death. Nevertheless, 
trial evidence is usually required before a new treatment is used routinely. In the absence of proven superiority by trials and the small survival benefit, this Task Force is of the opinion that no strict recommendations can be made, and prefers to merely offer guidance regarding the selection of patients for CRT-D or CRT-P, based on overall clinical condition, device-related complications and cost (Tables 17 and 18).

\section{Indication for concomitant implantable cardioverter defibrillator (cardiac resynchronization therapy and defibrillator)}

\begin{tabular}{|c|c|c|c|}
\hline Recommendations & Class $^{a}$ & Level $^{\mathrm{b}}$ & Ref. ${ }^{c}$ \\
\hline $\begin{array}{l}\text { I) When an ICD is planned, } \\
\text { a CRT is recommended when } \\
\text { indicated. }\end{array}$ & I & $\mathbf{A}$ & $\begin{array}{l}50,53 \\
54,60,62 \\
\text { (see also } \\
\text { sections } 3.2 \\
\text { and } 3.3 \text { ) }\end{array}$ \\
\hline $\begin{array}{l}\text { 2) When a CRT is planned, } \\
\text { implantation of CRT-D device } \\
\text { should be considered in } \\
\text { patients with clinical conditions } \\
\text { listed in Table I7. }\end{array}$ & Ila & $\mathbf{B}$ & $\begin{array}{c}46,55,57 \\
|3|\end{array}$ \\
\hline
\end{tabular}

$C R T=$ cardiac resynchronization therapy; CRT-D = CRT and defibrillator; $C R T-P=C R T$ and pacemaker; ICD = implantable cardioverter defibrillator ${ }^{a}$ Class of recommendation.

bLevel of evidence.

'Reference(s) supporting recommendation(s).

${ }^{\mathrm{d}}$ According to present ICD guidelines. ${ }^{\text {w69 }}$

Table 17 Clinical guidance to the choice of CRT-P or CRT-D in primary prevention

\begin{tabular}{|c|c|}
\hline Factors favouring CRT-P & Factors favouring CRT-D \\
\hline Advanced heart failure & Life expectancy $>$ I year \\
\hline $\begin{array}{c}\text { Severe renal insufficiency } \\
\text { or dialysis }\end{array}$ & $\begin{array}{c}\text { Stable heart failure, } \\
\text { NYHA II }\end{array}$ \\
\hline Other major co-morbidities & $\begin{array}{c}\text { Ischaemic heart disease } \\
\text { (low and intermediate MADIT } \\
\text { risk score) }\end{array}$ \\
\hline Frailty & Lack of comorbidities \\
\hline Cachexia & \\
\hline
\end{tabular}

CRT-D = cardiac resynchronization therapy and defibrillator; CRT-P = cardiac resynchronization therapy and pacemaker; MADIT = Multicentre Automatic Defibrillator Trial; NYHA = New York Heart Association.

\section{Indications for pacing in specific conditions}

\subsection{Pacing in acute myocardial infarction}

The incidence of new-onset AV block in patients with ST elevation myocardial infarction has decreased in the reperfusion
Table I 8 Comparative results of CRT-D vs. CRT-P in primary prevention

\begin{tabular}{|c|c|c|}
\hline $\begin{array}{c}\text { Mortality } \\
\text { reduction }\end{array}$ & $\begin{array}{c}\text { Similar level of } \\
\text { evidence but CRT-D } \\
\text { slightly better }\end{array}$ & $\begin{array}{c}\text { Similar level of } \\
\text { evidence but CRT-P } \\
\text { slightly worse }\end{array}$ \\
\hline Complications & Higher & Lower \\
\hline Costs & Higher & Lower \\
\hline
\end{tabular}

CRT-D = cardiac resynchronization therapy and defibrillator; CRT-P $=$ cardiac resynchronization therapy and pacemaker.

era from 5-7\% with thrombolytic therapy to $3.2 \%$ with primary percutaneous coronary intervention. ${ }^{132, w 174-w 179}$ High-degree AV block, associated with inferior wall infarction, is located above the His bundle in $90 \%$ of patients. ${ }^{\text {w18,w181 }}$ High-degree AV block associated with anterior infarction is more often located below the AV node. ${ }^{\text {w181 }}$ AV block complicating acute myocardial infarction most often resolves itself spontaneously within a few days or weeks, ${ }^{133-136}$ with only $9 \%$ of these patients requiring permanent pacing. ${ }^{132}$ Patients with AV block have a higher in-hospital and 30-day mortality than those with preserved AV conduction, irrespective of the site of the infarction. ${ }^{133}$ However, 30 days after the infarction, the mortality rates of patients with and without AV block are equal, indicating that both patient groups have the same long-term prognosis. ${ }^{132}$

Intraventricular conduction disturbances are more commonly developed in the setting of an anterior-anteroseptal infarction as a result of specific blood supply conditions. ${ }^{\text {w177,w182 }}$ Patients with $\mathrm{AV}$ block complicating an anterior infarction, and those with new-onset intraventricular conduction disturbances, have extremely high mortality (up to $80 \%$ ) due to the extensive myocardial necrosis. ${ }^{\text {} 182}$ Even if transient type 2 second- or third-degree heart block, associated with new-onset BBB, has been historically considered an indication for cardiac pacingeven in the absence of documentation of late development of $\mathrm{AV}$ block-there is no evidence of benefit of this strategy. ${ }^{135, \text { w183 }}$

\section{Clinical perspectives:}

- AV block complicating acute myocardial infarction most often resolves itself spontaneously within 2-7 days. Permanent cardiac pacing does not influence the prognosis of these patients and therefore is not recommended.

- In patients with anterior infarction, complicated by new-onset BBB and transient AV block, short- and long-term mortality is high irrespective of permanent pacing. There is no evidence that cardiac pacing improves the outcome. Since these patients often have HF and severe systolic dysfunction, it is the opinion of this Task Force that it seems more appropriate to evaluate the indications for CRT-D, rather than conventional antibradycardia pacing (see section 3.1). 


\section{Indications for permanent pacing}

\begin{tabular}{|l|c|c|c|}
\hline Recommendations & Class $^{\mathrm{a}}$ & Level $^{\mathrm{b}}$ & Ref. $^{\mathrm{C}}$ \\
\hline $\begin{array}{l}\text { I) In the rare cases in which } \\
\text { AV block becomes permanent, } \\
\text { cardiac pacing is indicated with } \\
\text { the same recommendations in } \\
\text { section 2.I. }\end{array}$ & I & C & - \\
\hline $\begin{array}{l}\text { 2) Cardiac pacing is not } \\
\text { indicated after resolution of } \\
\text { high degree or complete AV } \\
\text { block complicating the acute } \\
\text { phase of myocardial infarction. }\end{array}$ & III & B & 132-136 \\
\hline
\end{tabular}

$\mathrm{AV}=$ atrioventricular

${ }^{a}$ Class of recommendation.

b Level of evidence.

${ }^{c}$ Reference(s) supporting recommendation(s).

\subsection{Pacing after cardiac surgery, transcatheter aortic valve implantation, and heart transplantation}

Bradyarrhythmias are not uncommon after cardiac surgery, transcatheter aortic valve implantation (TAVI) and heart transplantion. Some bradyarrhythmias are transient and resolve themselves in the first days after surgery, others persist and permanent cardiac pacing has to be considered with the same recommendations as for unoperated patients. The clinically important question in managing post-operative bradyarrhythmias is related to the reasonable amount of time to allow for recovery of AV conduction or sinus node function after surgery before implanting a permanent PM.

\section{Cardiac surgery}

AV block may occur in 1-4\% of cases after cardiac surgery, in about $8 \%$ after repeat valve surgery and in $20-24 \%$ in interventions for calcific aortic stenosis or tricuspid valve replacement. ${ }^{\text {w184-w188 }}$ Sinus node dysfunction may occur after coronary artery bypass graft surgery, right lateral atriotomy or other transseptal superior approaches to the mitral valve. $^{\text {w185,w186 }}$ In clinical practice, an observation period of 5-7 days is usually applied before implanting a permanent PM, ${ }^{\text {w185 }}$ in order to allow regression of transient bradyarrhythmias. However, recovery may also occur later. At follow-up, the patients who are actually PM-dependent are $30-40 \%$ of those implanted for sinus node dysfunction and $65-100 \%$ of those implanted for AV block. ${ }^{\text {w185,w189 }}$ In case of complete AV block occurring in the first 24 hours after aortic and mitral valve surgery and persisting for $>48$ hours, resolution within the next 1 to 2 weeks is unlikely and earlier implantation of a PM can be considered in order to reduce post-operative length of stay. ${ }^{\text {w190,w191 }}$ The same approach appears reasonable for complete AV block with low rate of escape rhythm. ${ }^{\text {w185 }}$

\section{Transcatheter aortic valve implantation}

In a systematic review, ${ }^{\mathrm{w} 192}$ including retrospective series or registries, inclusive of 2047 patients from Europe and North America, the mean incidence of permanent PM implantation following TAVI was $14.2 \%$ (range $0-34 \%$; median 9.7\%): it was $20.8 \%$ (range $9.3-30.0 \%$ ) in five studies with the CoreValve prosthesis and $5.4 \%$ (range $0-10.1 \%$ ) in six studies with the Edwards-Sapien prosthesis. In a multi-centre registry, ${ }^{\text {w193 }}$ one third of patients undergoing a CoreValve transcatheter aortic valve implantation procedure required a PM within 30 days. In most cases, the PM was implanted within 5 days and, in three out of eight studies, within 24 hours. There is little evidence of recovery following complete $\mathrm{AV}$ block. ${ }^{\text {w192 }}$ New-onset persistent LBBB is common following TAVI, but the significance of the finding and the follow-up required is unclear. ${ }^{\text {w192 }}$ Independent predictors of PM requirement following TAVI include use of the CoreValve prosthesis and evidence of conduction system dysfunction, either pre-existing RBBB or AV block at the time of TAVI. Although TAVI patients usually meet the criteria for CRT in patients with conventional indication for anti-bradycardia pacing (see section 4.3.2), there is limited experience of CRT pacing in TAVI patients.

\section{Heart transplantation}

Sinus node dysfunction is common and leads to permanent PM implantation in $8 \%$ of patients. ${ }^{\text {w185 }}$ Possible causes of sinus node dysfunction include surgical trauma, sinus node artery damage, or ischaemia and prolonged cardiac ischaemic times. ${ }^{\text {w194,w195 }}$ AV block is less common and is probably related to inadequate preservation of the donor

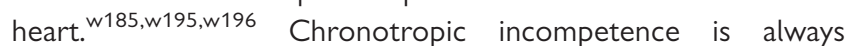
present following standard orthotopic heart transplantation, as a result of loss of autonomic control. Since sinus node and AV node function improve during the first few weeks after transplantation, an observation period before PM implantation may allow spontaneous improvement of bradycardia. ${ }^{\text {w197 }}$ There is a general consensus that patients in whom symptomatic bradycardia persists after the third post-operative week, require permanent PM implantation. DDDR mode with minimized ventricular pacing or AAIR in the case of intact AV nodal conduction are recommended. ${ }^{\mathrm{w} 195}$

\section{Clinical perspectives:}

- If significant bradyarrhythmia does not resolve in the suggested observation period after cardiac surgery, TAVI or heart transplantation, permanent cardiac pacing is indicated with the same recommendations as in section 2.2. However, in case of high-degree or complete AV block with low rate of escape rhythm, this observation period can be shortened since resolution is unlikely. For sinus node dysfunction in heart transplanted patients, the period of observation could be several weeks. 
Pacing after cardiac surgery, transcatheter aortic valve implantation and heart transplantation

\begin{tabular}{|c|c|c|}
\hline Recommendations & Class $^{a}$ & Level $^{\text {b }}$ \\
\hline $\begin{array}{l}\text { I) High degree or complete AV block } \\
\text { after cardiac surgery and TAVI. A period } \\
\text { of clinical observation up to } 7 \text { days is } \\
\text { indicated in order to assess whether the } \\
\text { rhythm disturbance is transient and resolves. } \\
\text { However, in case of complete AV block with } \\
\text { low rate of escape rhythm this observation } \\
\text { period can be shortened since resolution is } \\
\text { unlikely. }\end{array}$ & I & C \\
\hline $\begin{array}{l}\text { 2) Sinus node dysfunction after cardiac } \\
\text { surgery and heart transplantation. } \\
\text { A period of clinical observation from } \\
5 \text { days up to some weeks is indicated in order } \\
\text { to assess if the rhythm disturbance resolves. }\end{array}$ & I & C \\
\hline $\begin{array}{l}\text { 3) Chronotropic incompetence after } \\
\text { heart transplantation. Cardiac pacing should } \\
\text { be considered for chronotropic } \\
\text { incompetence impairing the quality of life late } \\
\text { in the post-transplant period. }\end{array}$ & Ila & C \\
\hline $\begin{array}{l}\text { s of recommendation. } \\
\text { l of evidence. } \\
\text { rence(s) supporting recommendation(s). }\end{array}$ & & \\
\hline
\end{tabular}

\subsection{Pacing and cardiac resynchronization therapy in children and in congenital heart disease}

Despite many similarities in pacing indications between young people and adults, several differences justify the writing of a separate, dedicated chapter. Since children are paced for a lifetime, they are prone to a higher incidence of long-term adverse events and are at high risk of experiencing the adverse consequences of cardiac stimulation at a non-optimal site. Because of a small body size, the presence of a congenital defect with a right-to-left shunt, or postoperative absence of transvenous access to the target chamber, children often need to be permanently paced epicardially. ${ }^{\text {w198- }}$ w200 Children's higher activity levels lead to greater stress on device hardware and their growth expectancy leads to higher incidence of lead dislodgement or fracture in follow-up. Concerns have been voiced regarding the long-term performance of endocardial leads in children, given the high incidence of abandonment, potential

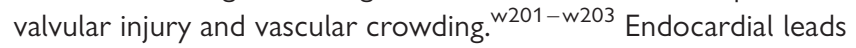
are contra-indicated in patients with right-to-left shunt because of the risk of systemic thromboemboli. ${ }^{\text {w200 }}$ Therefore, in young patients, it seems preferable to postpone endocardial pacing to minimize the risks associated with the presence of multiple intracardiac leads. When allowed during the surgical intervention, attempts should be made to stimulate either the left or the systemic ventricle, ${ }^{\text {w204 }}$ although studies looking at chronic results of LV or systemic pacing are warranted.

\section{Congenital AV block}

The decision to proceed with the implantation of a permanent PM in patients suffering from congenital AV block is strongly influenced by the awareness that (i) Adams-Stokes attacks and HF might develop in children, adolescents or adults of any age and (ii) the first manifestation of congenital AV block might be sudden death, without prodromal symptoms and in the absence of manifestations of underlying heart disease. ${ }^{\text {w205-w207 }}$ Consequently, any unnecessary postponement of permanent pacing increases the risk of cardiac remodelling and sudden death.

\section{Indications for cardiac pacing (Recommendations 1 and 2)}

The development of syncope or pre-syncope, HF or chronotropic incompetence limiting the level of physical activity justifies the implantation of a PM. ${ }^{\text {w205-w209 }}$ Patients presenting with ventricular dysfunction are also candidates for permanent pacing which, if instituted before the onset of symptoms, is likely to preserve cardiac function. ${ }^{\text {w208,w210 }}$ Prophylactic pacing is indicated in asymptomatic patients who are at risk of syncope or sudden death, heralded by bradycardia, long pauses greater than three times the cycle length during ventricular escape rhythm, a wide QRS complex, a prolonged QT interval or complex ventricular ectopy. ${ }^{\text {w209-w211 }}$ A subset of patients paced for isolated congenital AV block develops a dilated cardiomyopathy requiring close longterm surveillance of the proper functions of the pacing system, as well as their ventricles. ${ }^{\text {w212-w215 }}$

Even if the quality of evidence is modest, there is a strong consensus that patients with third- or second-degree (Mobitz II) AV block must receive permanent cardiac pacing therapy if symptomatic or with risk factors. In asymptomatic patients without risk factors, there is divergence of opinion on the benefit of cardiac pacing.

\section{Post-operative atrioventricular block}

In patients with congenital heart disease, post-operative AV conduction block complicates $1 \%$ to $3 \%$ of cardiac operations. ${ }^{\text {w216 }}$ The risk is greatest for the surgical repair of ventricular septal defects. Spontaneous resolution of complete AV block in the early post-operative period can occur, usually within 10 days after the operation. ${ }^{137-141}$ The prognosis for patients whose AV conduction returns to normal is favourable. In contrast, the prognosis for non-paced patients is very poor. In a large meta-analysis, residual bifascicular block that persisted after the disappearance of transient post-surgical complete heart block was associated with a high incidence of late recurrence of AV block or sudden death (29\%). ${ }^{138}$ Post-operative HV interval determination may help to assess the risk of late-onset AV block in patients with residual conduction disorder (long PR interval, bifascicular block). ${ }^{\text {2217 }}$

\section{Indications for cardiac pacing (Recommendations 3 and 4)}

There is modest evidence and strong consensus that patients with persistent third- or second-degree AV block must receive permanent cardiac pacing therapy. The evidence is modest and the consensus is weak for patients who have persistent bifascicular block (with or without PR interval prolongation) associated with transient AV block or with permanent prolonged PR interval. 


\section{Sinus node disease and bradycardia-tachycardia syndrome}

In children, sinus node dysfunction might precede or follow reparative cardiac surgery involving the atria, though it is also observed in patients treated with anti-arrhythmic drugs and in patients with an otherwise normal heart. ${ }^{\text {w218,w219 }}$ Contrary to AV block, sinus node dysfunction is not associated with increased mortality.

\section{Indications for cardiac pacing (Recommendations 5 and 6)}

The occurrence of symptomatic sinus node disease justifies the implantation of a PM if competing causes have been ruled-out after extensive examination. ${ }^{\text {w220,w221 }}$ Chronotropic incompetence has been correlated with the late development of postoperative atrial flutter. ${ }^{\text {w222 }}$ Pacing to restore chronotropic competence may prevent late post-operative atrial flutter and reduce exercise intolerance, especially late after Mustard, Senning or Fontan procedures. Pacing can also be used to treat congestive $\mathrm{HF}$ or fatigue and to prevent the development of supraventricular arrhythmias. ${ }^{\text {223,w224 }}$ The evidence of benefit is lacking for young, asymptomatic patients, with a heart rate $<40 \mathrm{bpm}$ or $>3$ sec pauses. ${ }^{\text {w218-w221 }}$

There is sufficient evidence and large consensus that cardiac pacing is beneficial in symptomatic sinus node disease. The evidence is modest and the consensus is weak for patients who have less severe forms of sinus node disease.

\section{Cardiac resynchronization in congenital heart disease}

Evidence of benefit from CRT is limited to case reports, retrospective analyses of heterogeneous populations, small crossover trials conducted in the immediate post-operative period and

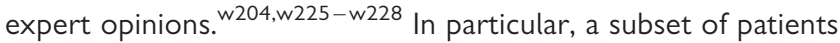
paced in RV for isolated congenital AV block develops a dilated cardiomyopathy. In these patients, single-site LV pacing is particularly attractive for children and young adults. A recent European multicentre study showed that, during a mean follow-up of 5.4 years, pacing from the RV outflow tract/lateral RV caused a significant decrease in LV function, whereas LV apex/LV mid-lateral wall pacing was associated with preserved LV function. ${ }^{\text {w229 }}$ For the purpose of single-site LV pacing, either epicardial implantation or implantation via the coronary sinus may be used (Web Figure 12). However, the evidence is not great enough and this Task Force cannot make any specific recommendation.

\section{Clinical perspectives:}

- An individualized evaluation of the benefits vs. potential complications of pacemaker implantation is recommended in children, taking into consideration the cardiac and venous anatomy, patient size and growth expectancy.

- The decision to implant a pacemaker in children is done in collaboration with paediatric cardiologists and should preferably be done in a specialized centre.

- Single-site LV pacing, instead of RV pacing, is an attractive mode of pacing in order to preserve cardiac function, but it requires further evidence.
Indications for pacing therapy in paediatric patients and congenital heart disease

\begin{tabular}{|c|c|c|c|}
\hline Recommendations & Class $^{a}$ & Level $^{b}$ & Ref. $^{c}$ \\
\hline $\begin{array}{l}\text { I) Congenital AV block. Pacing } \\
\text { is indicated in high degree and } \\
\text { complete AV block in symptomatic } \\
\text { patients and in asymptomatic } \\
\text { patients with any of the following } \\
\text { risk conditions: ventricular } \\
\text { dysfunction, prolonged QTc } \\
\text { interval, complex ventricular } \\
\text { ectopy, wide QRS escape rhythm, } \\
\text { ventricular rate }<50 \text { b.p.m., } \\
\text { ventricular pauses >three-fold the } \\
\text { cycle length of the underlying } \\
\text { rhythm. }\end{array}$ & I & C & - \\
\hline $\begin{array}{l}\text { 2) Congenital AV block. Pacing } \\
\text { may be considered in } \\
\text { asymptomatic patients with high } \\
\text { degree and complete AV block in } \\
\text { absence of the above risk } \\
\text { conditions. }\end{array}$ & Ilb & C & - \\
\hline $\begin{array}{l}\text { 3) Postoperative AV block in } \\
\text { congenital heart disease. } \\
\text { Permanent pacing is indicated for } \\
\text { postoperative advanced second } \\
\text { degree or complete AV block } \\
\text { persisting }>10 \text { days. }\end{array}$ & I & $\mathbf{B}$ & $|37-| 4 \mid$ \\
\hline $\begin{array}{l}\text { 4) Postoperative AV block in } \\
\text { congenital heart disease. } \\
\text { Permanent pacing should be } \\
\text { considered for persistent, } \\
\text { asymptomatic post-surgical } \\
\text { bifascicular block (with or without } \\
\text { PR prolongation) associated with } \\
\text { transient, complete AV block. }\end{array}$ & Ila & C & - \\
\hline $\begin{array}{l}\text { 5) Sinus node disease. } \\
\text { Permanent pacing is indicated for } \\
\text { symptomatic sinus node disease, } \\
\text { including brady-tachy syndrome, } \\
\text { when a correlation between } \\
\text { symptoms and bradycardia is } \\
\text { judged to be established. }\end{array}$ & I & C & - \\
\hline $\begin{array}{l}\text { 6) Sinus node disease. } \\
\text { Permanent pacing may be useful } \\
\text { for asymptomatic resting heart } \\
\text { rate }<40 \text { b.p.m. or ventricular } \\
\text { pauses lasting }>3 \mathrm{sec} \text {. }\end{array}$ & Ilb & C & - \\
\hline
\end{tabular}

$\mathrm{AV}=$ atrioventricular

${ }^{a}$ Class of recommendation.

bLevel of evidence.

'Reference(s) supporting recommendation(s)

\subsection{Pacing in hypertrophic cardiomyopathy \\ Bradyarrhythmia}

Atrioventricular block is uncommon in hypertrophic cardiomyopathy (HCM), but in context, can suggest specific aetiologies (for example, PRKAG2 gene mutations, Anderson-Fabry disease and amyloidosis). Atrioventricular block in patients with HCM should be treated in accordance with the general recommendations of this Guideline (see section 2.1 and 2.2).

Chronotropic incompetence during upright exercise testing is more common in patients with advanced disease and is an important determinant of exercise performance. ${ }^{\text {w230,w231 }}$ The role of pacing to 
improve exercise capacity in this context has not been formally assessed.

\section{Treatment of left ventricular outflow tract obstruction (Recom- mendation 1)}

In patients with symptoms caused by left ventricular outflow tract obstruction, treatment options include negative inotropic drugs, surgery, septal alcohol ablation and sequential AV pacing. Approximately $60-70 \%$ of patients improve with medical therapy alone. For the remainder, surgery (septal myectomy) or septal alcohol ablation can, in appropriately selected patients and in experienced centres, improve functional status with a similar procedural mortality, but a higher rate of permanent PM implantation following alcohol ablation. ${ }^{\text {232-w234 }}$ The risk of AV block is highest in patients with preexisting conduction disease and prophylactic permanent pacing before intervention has been advocated. ${ }^{142}$

Right ventricular apical pacing alone has also been advocated as a therapy for HCM. Pacing creates pre-excitation of the RV apex, which changes the ventricular contraction pattern and creates regional dyssynchrony. The result is late activation of the basal part of the septum and decreased LV contractility, which reduce systolic anterior motion of the mitral valve and the severity of $L V$ outflow tract obstruction. ${ }^{\text {w235 }}$ Pre-excitation of the RV apex is achieved by short $A V$ delay DDD pacing. The sensed AV delay needs to be shorter than the spontaneous PR interval in order to achieve RV pacing. However, short intervals may interfere with atrial emptying and result in reduced cardiac output. ${ }^{143, w 236}$ In order to be successful, AV delay values short enough to reduce the gradient but long enough to preserve LV filling (measured by echocardiography) are required; in general this is achieved with a resting $A V$ interval of $100 \pm 30 \mathrm{~ms}^{143}$ A dynamic AV interval can also be programmed to ensure complete ventricular capture during exercise. The upper rate limit should be programmed higher than the fastest sinus rate achievable during exercise, to ensure permanent ventricular pacing even during brisk exercise. In some patients with a very short PR interval, AV nodal ablation has been advocated as a method of achieving an optimal AV delay, but this is not recommended in this Guideline. ${ }^{\text {w237 }}$

Reduction in outflow tract gradients and inconsistent effects on symptoms and quality of life have been demonstrated in three small randomized, placebo-controlled studies of DDD vs. AAl pacing and in long-term observational studies. ${ }^{143-148}$ In general, the magnitude of gradient reduction is also less than with surgery or alcohol septal reduction. In one trial, a retrospective subgroup analysis suggested that older patients ( $>65$ years of age) are more likely to benefit.

Finally, a significant number of patients with HCM receive an ICD for primary or secondary prevention. Implanting a dual-chamber device and programming DDD pacing with short adapted AV delay may alleviate obstruction and prevent the need for complementary and risky procedures such as surgery or alcohol ablation.

There is sufficient evidence to suggest that permanent AV sequential pacing with short $A V$ interval can reduce outflow tract obstruction and improve symptoms in selected patients who are unsuitable for - or unwilling to consider-invasive septal reduction therapies.

\section{Cardiac resynchronization therapy}

Regional heterogeneity of contraction and relaxation is well recognized in HCM and the presence of dyssynchrony has been shown to be a marker of poor prognosis. ${ }^{\text {w238 }}$ Several case reports and a single centre cohort study have suggested that CRT pacing alleviated HF symptoms and, in patients with end-stage HCM, was associated with reverse remodelling of the left atrium and ventricle. ${ }^{\text {w239-w242 }}$ Small cohort studies have also examined CRT as a treatment for LV outflow tract obstruction, but its superiority over conventional $\mathrm{RV}$ pacing is not established. ${ }^{\mathrm{w} 243, \mathrm{w} 244}$

\section{Clinical perspectives:}

- In general, patients with drug-refractory symptoms caused by LV outflow tract obstruction should be considered for surgery or alcohol ablation.

- In patients with LV outflow tract obstruction treated with pacemaker or dual-chamber ICD, a short AV interval programming is crucial. The objective is to achieve maximum $R V$ apical pre-excitation without compromising LV diastolic filling.

- In the absence of LV outflow tract obstruction, AV block complicating HCM should be treated in accordance with general recommendations of this Guideline.

- Patients with HCM can develop systolic dysfunction and symptoms of heart failure. In the absence of randomized trials, CRT may be considered in individual cases in which there is some evidence for systolic ventricular impairment and dyssynchrony (see section 3.1).

\section{Indication for cardiac pacing in patients with hypertrophic cardiomyopathy}

\begin{tabular}{|c|c|c|c|}
\hline Recommendations & Class $^{\mathrm{a}}$ & Level $^{b}$ & Ref. $^{c}$ \\
\hline $\begin{array}{l}\text { I) Left ventricular outflow } \\
\text { tract obstruction. Sequential } \\
\text { AV pacing with short AV interval } \\
\text { may be considered in selected } \\
\text { patients with resting or } \\
\text { provocable LV outflow tract } \\
\text { obstruction and drug-refractory } \\
\text { symptoms who: } \\
\text { a) have contraindications for } \\
\text { septal alcohol ablation or septal } \\
\text { myectomy; }\end{array}$ & Illb & B & $\mid 42-148$ \\
\hline $\begin{array}{l}\text { or } \\
\text { b) or are at high risk of } \\
\text { developing heart block following } \\
\text { septal alcohol ablation or septal } \\
\text { myectomy. }\end{array}$ & Ilb & C & - \\
\hline $\begin{array}{l}\text { 2) For patients in whom there is } \\
\text { an indication for an ICD, a } \\
\text { dual-chamber ICD should be } \\
\text { considered }\end{array}$ & Ila & C & - \\
\hline
\end{tabular}

$\mathrm{AV}=$ atrioventricular

${ }^{\mathrm{a}}$ Class of recommendation.

bLevel of evidence.

${ }^{\mathrm{c}}$ Reference(s) supporting recommendation(s).

\subsection{Pacing in rare diseases}

Rare diseases (population prevalence less than 1 in 2000) affect 6$8 \%$ of the European population. Some, such as LQTS or familial AV block affect only the heart, whereas others are multi-system disorders with variable cardiac involvement. A full discussion of all rare disease relevant to the heart is beyond the scope of this Guideline, but some of the more commonly encountered disorders that 
cause clinically significant arrhythmia are listed in Web Table 19. Bradyarrhythmias in patients with inherited rare diseases should be treated in accordance with general recommendations of this Guideline (see sections 2.1 and 2.2).

\subsubsection{Long QT syndrome}

The trigger for most episodes of life-threatening arrhythmias is a sudden increase in sympathetic activity, mediated by left-sided cardiac sympathetic nerves. Beta-blockers are the mainstay of drug treatment in patients with LQT1 and LQT2 as large registries indicate that they reduce mortality, even in asymptomatic mutation carriers. When patients continue to experience symptoms in spite of ß-blockade, left stellate ganglionectomy or ICDs should be consider-

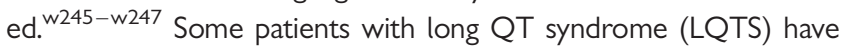
pause-dependent ventricular arrhythmia, particularly those with LQT3, in whom ventricular arrhythmias often occur at rest or during sleep. In the past, PMs have been advocated in patients with pausedependent ventricular arrhythmia, but data from small observational series suggest that pacing reduces syncopal events but does not prevent sudden cardiac death. ${ }^{\text {w248-w250 }}$ Therefore, an ICD is preferable in symptomatic patients with LQT3 or pause-dependent ventricular arrhythmia. Symptomatic infants may be an exception, as pacing with full dose ß-blockers might postpone the need for an ICD. Pacing algorithms in patients with an ICD can also help to prevent shocks.

The current role of PM therapy in long QT syndrome is very limited. An $I C D$ (with active pacing) is preferable in patients with symptoms unresponsive to B-blocker therapy or pause-dependent ventricular arrhythmia according to current ICD guidelines to which we refer.

\subsubsection{Muscular dystrophies}

Muscular dystrophies are a heterogeneous group of inherited disorders, characterized by progressive skeletal muscle wasting and weak-

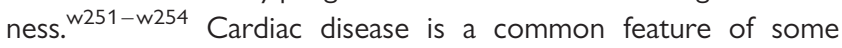
muscular dystrophies.

\section{Laminopathies}

Data from several series, ${ }^{\text {w255-w258 }}$ a meta-analysis and a multi-centre European registry indicate that cardiac disease caused by mutations in the lamin AC gene (LMNA) has a poor prognosis, with a $25 \%$ mortality from sudden cardiac death and progressive HF by the age of 50

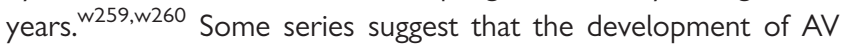
block is associated with poor outcomes, but pacing therapy alone does not prevent sudden cardiac death.

\section{Emery-Dreifuss muscular dystrophy}

A typical finding of $X$-linked recessive Emery-Dreifuss muscular dystrophy (EDMD) is atrial standstill or atrial paralysis, related to replacement of atrial myocardium by fibrous tissue. ${ }^{\text {w261 }}$ Pacing is indicated at the first appearance of bradyarrhythmias or conduction disturbances, in general before the age of 30 years. Following implantation of a PM, the incidence of sudden death appears low, but the risk of stroke remains high because of atrial standstill and AF. ${ }^{\text {w262 }}$

\section{Myotonic dystrophy}

In the heart, myotonic dystrophy type 1 (DM1) causes progressive conduction disease, ventricular arrhythmia and systolic impairment. In a recent systematic review of 18 studies (1828 patients), ventricular premature beats were the most prevalent arrhythmia (14.6\%) followed by AF or atrial flutter (5.0\%) and non-sustained ventricular

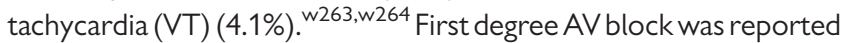
in $25-30 \%$ of patients and QRS duration $>120 \mathrm{~ms}$ in $19.9 \%$. The prevalence of PM or ICD implants in 13 cross-sectional studies $(n=1234)$ was 4.1 and $1.1 \%$, respectively. The probability of receiving a PM or ICD was 1.0 and $0.2 \%$ per follow-up year, respectively. Sudden cardiac death accounts for up to $33 \%$ of all deaths. The mechanism has been assumed to be progressive conduction disease, but reports of sudden death in patients with pacemakers and spontaneous or inducible VT suggest that ventricular arrhythmias might explain some cases. ${ }^{\text {w265,w266 }}$

Development of criteria for pacemaker and ICD implantation is challenging because of the small size and heterogeneity of published cohort studies and the confounding effect of progressive neuromuscular disease on survival. A number of clinical risk markers have been proposed including: age at symptom onset, severity of muscular involvement, number of CTG trinucleotide repeats, supraventricular arrhythmias, AV conduction disturbances, abnormal signal-averaged ECG and reduced heart rate turbulence. ${ }^{\text {w267-w271 }} \mathrm{A}$ small study has suggested that prolongation of the HV interval (>70 ms) at invasive EPS is predictive of complete AV block. ${ }^{\text {} 268}$ In a small study of serial invasive measurement of HV intervals, evidence of new conduction disease on a resting ECG and/or signal-averaged ECG were associated with subsequent lengthening of infrahissian conduction. ${ }^{\text {w267 }}$ In a large retrospective, single centre registry of 486 genetically confirmed patients with conduction disease (PR interval $>200 \mathrm{~ms}$ and/ or QRS duration $>100 \mathrm{~ms}$ ), ${ }^{\text {w271 }}$ a comparison was made between those who underwent an invasive EPS followed by a PM implantation if their HV interval was $>70 \mathrm{~ms}$, and those that did not. There was no significant difference in overall mortality over a median follow-up of 7.4 years. When adjusted for baseline characteristics, there was borderline significance in overall survival in favour of the invasive strategy, largely due to a significant reduction in adjusted survival from sudden cardiac death. Overall patients with conduction disease had a poorer survival, irrespective of EPS, compared with those without. These data provide incremental evidence in support of EPS in patients with conduction disease, but the small improvement in overall survival means that the clinical significance of this study is unclear.

\section{Desminopathies}

Desmin-related myopathy is characterized by progressive skeletal muscle weakness, cardiomyopathy and cardiac conduction disease, with variable age of disease onset and rate of progression. ${ }^{\text {w270 }}$ In a recent meta-analysis of 159 carriers of desmin mutation, ${ }^{\text {w269 }} 50 \%$ had cardiomyopathy and approximately $60 \%$ had cardiac conduction disease or arrhythmias. Twenty-five per cent of carriers died at a mean age of 49 years. Sudden cardiac death occurred in two patients with a pacemaker.

\subsubsection{Mitochondrial cytopathies}

Supraventricular and ventricular arrhythmias and conduction defects, are the most common cardiac presentations in patients with mitochondrial disease. Cardiac conduction disease is the key feature of the Kearns-Sayre disease. Ventricular arrhythmias and sudden death are also reported, often in association with HCM in adults and children. ${ }^{\text {w272-w274 }}$ 


\subsubsection{Metabolic disorders}

In adults, one of the most common metabolic disorders is AndersonFabry disease, an $\mathrm{X}$-linked lysosomal storage disorder that causes HCM in middle and later life. ${ }^{\text {275 }}$ In the later stages of the disease, sino-atrial dysfunction and AV conduction disturbance can necessitate PM implantation. QRS duration is a predictor for future PM implantation, ${ }^{\text {} 276}$ but in the absence of prospective trials we suggest adherence to conventional pacing and ICD indications, with close monitoring of patients with ECG evidence for conduction disease.

\section{Clinical perspectives:}

- Numerous rare genetic disorders can cause conduction disease but, for most, there is little evidence for disease-specific treatments, except possibly for laminopathies, in which early ICD might be considered, and myotonic dystrophy, in which PM might be considered if a prolonged HV interval is detected at EPS. Therefore conventional pacing/ICD indications should be applied in most cases.

\subsection{Pacing in pregnancy}

This topic has been recently covered by the ESC Guidelines on the management of cardiovascular diseases during pregnancy. ${ }^{\text {w277 }}$ Vaginal delivery carries no extra risks in a mother with congenital complete heart block, unless contra-indicated for obstetric reasons. For women who have a stable, narrow, complex junctional escape rhythm, PM implantation can be deferred until after delivery. ${ }^{\text {13,w278,w279 }}$ However, women with complete heart block who exhibit a slow, wide QRS complex escape rhythm should undergo PM implantation during pregnancy. ${ }^{\text {} 277}$ The risks of PM implantation are generally low and can be performed safely, especially if the foetus is beyond 8 weeks' gestation. A PM for the alleviation of symptomatic bradycardia can be implanted at any stage of pregnancy using echo guidance or electro-anatomic navigation avoiding fluoroscopy. ${ }^{\text {2278,w280-w282 }}$

\section{Pacing in pregnancy}

\begin{tabular}{l|l|c|}
\hline Recommendations & Class $^{\mathrm{a}}$ & Level $^{\mathrm{b}}$ \\
\hline $\begin{array}{l}\text { Implantation of permanent pacemakers } \\
\text { (preferably one chamber) should be } \\
\text { considered with echocardiographical } \\
\text { guidance, especially if the foetus is beyond } \\
8 \text { weeks gestation in selected women with } \\
\text { symptomatic complete AV block. }\end{array}$ & & \\
\hline
\end{tabular}

\subsection{Pacing for first-degree atrioventricular block (haemodynamic)}

First degree AV (1st AV) block is commonly considered a benign condition. However, a very long PR interval may exacerbate symptoms, especially during moderate or mild exercise. A marked 1st AV block, PR interval $>0.3 \mathrm{~s}$, in rare cases may result in symptoms similar to those in the PM syndrome. With a prolonged PR interval, atrial systole occurs too early in diastole, resulting in an ineffective or decreased contribution of the atrial systole to cardiac output. Echocardiographical studies show a fusion of the $E$ and $A$ waves in patients with a long PR interval, resulting in a shortening of the LV filling time and a diastolic mitral regurgitation. As a consequence, the increase of pulmonary capillary wedge pressure causes dyspnoea and retrograde blood flow in the jugular veins, leading to a sensation of fullness in the neck and palpitations described as 'pauses' or 'strong beats'. This deleterious effect is more marked in patients with LV dysfunction and/or HF. ${ }^{\text {w14-w16,w283 }}$

Some uncontrolled and non-randomized studies have suggested that a reduction of the $\mathrm{AV}$ timing using conventional DDD PM would improve symptoms and patients' functional status, especially in patients with preserved LV function. The improvement with DDD pacing is directly linked to the improvement in LV filling time. $^{\text {w14,w16 }}$

There are some potentially harmful consequences of conventional DDD pacing. The first one is that permanent $R V$ pacing may enhance LV dysfunction. To avoid this potential effect, biventricular pacing could be considered, but there is a definitive lack of data to support this concept, especially in patients with narrow QRS and/ or preserved LV function. The systematic use of biventricular pacing is not recommended at this time for this particular indication in the absence of other CRT indications. The second potential deleterious effect is the risk of functional atrial under-sensing due to the shift of the $P$ wave in the post-ventricular atrial refractory period, especially with fast heart rate; an exercise test would be helpful to ensure an adequate programming of the PM when the patient is exercising. ${ }^{\text {w283,w284 }}$

\section{Indication for pacing for first-degree atrioventricular block}

\begin{tabular}{l|l|l|}
\hline Recommendations & Class $^{\mathbf{a}}$ & Level $^{\mathbf{b}}$ \\
\hline $\begin{array}{l}\text { Permanent pacemaker implantation should } \\
\text { be considered for patients with persistent } \\
\text { symptoms similar to those of pacemaker } \\
\text { syndrome and attributable to first-degree } \\
\text { atrioventricular block (PR }>0.3 \mathrm{~s})\end{array}$ & Ila & C \\
\hline
\end{tabular}

\subsection{Algorithms for prevention and termination of atrial arrhythmias by pacing}

The rationale for the use of specific pacing algorithms is to avoid bradycardia and large atrial cycle length variations, which are thought to trigger atrial tachyarrhythmias (AT). Specific algorithms have included rate-adaptive pacing, which periodically assesses the underlying intrinsic rate to pace just above it, elevation of the pacing rate after spontaneous atrial ectopy, transient high-rate pacing after mode switch episodes and increased post-exercise pacing to prevent an abrupt drop in heart rate. In addition, some 
Table 20 Summary of randomized clinical studies of specific algorithms for prevention and termination of atrial tachyarrhythmias in patients with conventional brady indications and atrial tachyarrhythmias/fibrillation

\begin{tabular}{|c|c|c|c|c|c|}
\hline Trial & Study design & Algorithm/s & $\begin{array}{l}\text { No. of } \\
\text { patients }\end{array}$ & Effect on AF burden & Clinical result \\
\hline $\mathrm{ADOPT}^{|5|}$ & Parallel & Rate-adaptive pacing at high rest rate & 288 & $\begin{array}{l}25 \% \text { decrease in } \\
\text { symptomatic AF burden } \\
(P=0.005)\end{array}$ & $\begin{array}{l}\text { No change in quality of life, } \\
\text { hospitalizations and adverse } \\
\text { events }\end{array}$ \\
\hline PIRAT ${ }^{155}$ & Cross-over & Post-mode switch overdrive pacing & 37 & $\begin{array}{l}\text { No change in AT episodes, } \\
\text { AT burden }\end{array}$ & $\begin{array}{l}\text { No change in number of } \\
\text { symptoms and quality of life }\end{array}$ \\
\hline ATTEST $^{157}$ & Parallel & $\begin{array}{l}\text { Atrial preference } \\
\text { Atrial rate stabilization } \\
\text { Post-mode switch overdrive pacing } \\
\text { ATP therapy }\end{array}$ & 324 & $\begin{array}{l}\text { No difference in AT/AF } \\
\text { burden and frequency }\end{array}$ & Not assessed \\
\hline PIPAF $^{150}$ & Cross-over & $\begin{array}{l}\text { SR overdrive } \\
\text { Post-extrasystolic pause suppression } \\
\text { Acceleration after premature atrial beats }\end{array}$ & 28 & $\begin{array}{l}\text { No change in mode-switch } \\
\text { episodes and \% } \mathrm{A} / \mathrm{V} \text { pacing }\end{array}$ & $\begin{array}{l}\text { No difference in symptom } \\
\text { score }\end{array}$ \\
\hline PAFS 159 & Cross-over & $\begin{array}{l}\text { Rate-smoothing, } \\
\text { Rate stabilization }\end{array}$ & 182 & No change & $\begin{array}{l}\text { No change in episode number, } \\
\text { quality of life, or symptoms }\end{array}$ \\
\hline AOPS 156 & Cross-over & Rate-adaptive pacing at high rest rate & 99 & $\begin{array}{l}\text { No change in mode-switch } \\
\text { episodes }\end{array}$ & $\begin{array}{l}\text { No change in symptoms of } \\
\text { arrhythmia }\end{array}$ \\
\hline $\mathrm{POT}^{158}$ & Cross-over & $\begin{array}{l}\text { Atrial preference } \\
\text { Atrial rate stabilization } \\
\text { Post-mode switch overdrive pacing } \\
\text { ATP therapy }\end{array}$ & 85 & $\begin{array}{l}72 \% \text { decrease in AF burden } \\
\text { with preventive algorithms, } \\
\text { no further reduction with } \\
\text { ATP therapy }\end{array}$ & Not assessed \\
\hline SAFARI ${ }^{152}$ & Parallel & $\begin{array}{l}\text { Combination of six triggered and continuous } \\
\text { overdrive prevention pacing therapies }\end{array}$ & 240 & $\begin{array}{l}\text { Slight reduction in } \mathrm{AF} \\
\text { burden }(0.08 \mathrm{~h} / \text { day, } \\
P=0.03)\end{array}$ & Not assessed \\
\hline ASSERT ${ }^{153,154}$ & Parallel & Atrial overdrive pacing & 2343 & $\begin{array}{l}\text { No difference in } \\
\text { device-detected AT }\end{array}$ & $\begin{array}{l}\text { No difference in symptomatic } \\
\text { and asymptomatic AT. } \\
\text { No difference in stroke, } \\
\text { hospitalization and death }\end{array}$ \\
\hline
\end{tabular}

$\mathrm{AF}=$ atrial fibrillation; $\mathrm{AT}=$ atrial tachyarrhythmias; $\mathrm{ATP}=$ anti-tachycardia pacing; $\mathrm{SR}=$ sinus rhythm.

devices have incorporated atrial anti-tachycardia pacing (ATP) algorithms (high rate ramp and burst pacing) for termination of atrial tachycardia or atrial flutter that might degenerate into AF.

After the pivotal multi-centre study of Israel et al., ${ }^{149}$ several randomized trials were carried out, which showed that preventive algorithms had minimal or no incremental benefits for the prevention of AF (Table 20). ${ }^{150-159}$ In the largest of these trials, ${ }^{153,154} 2343$ patients with hypertension and no history of AF, in whom a PM or defibrillator had been implanted, were randomly assigned to receiveor not to receive - continuous atrial overdrive pacing, which did not prevent AF or a clinical outcome of stroke, systemic embolism, myocardial infarction, death from vascular causes, or hospitalization for HF. Similarly, some RCTs failed to show a clinical benefit from antitachycardia pacing algorithms (ATP). ${ }^{157,158}$ Similar results have been demonstrated in ICD patients. ${ }^{\text {w285,w286 }}$ In a pooled analysis of four ATP studies, Gillis et al. suggested that ATP may be valuable in a minority (about $30 \%$ ) of patients with particularly organized AT, in whom ATP is documented to be able to terminate $>60 \%$ of episodes. ${ }^{\text {w287 }}$

Finally, no consistent data from large randomized trials support the use of alternative single-site atrial pacing, ${ }^{288, w 289}$ dual-site right atrial pacing, ${ }^{\text {w290 }}$ or bi-atrial pacing, ${ }^{\text {w291 }}$ alone or in association with algorithms for prevention and termination of AT.

There is strong evidence that algorithms designed to prevent AF have no incremental benefits for the prevention of AF; further trials are unlikely to change the confidence in the estimate of effect.

\section{Indication for prevention and termination of atrial tachyarrhythmias}

\begin{tabular}{|l|c|c|c|}
\hline Recommendations & Class $^{\text {a }}$ & Level $^{\text {b }}$ & Ref. $^{\text {c }}$ \\
\hline $\begin{array}{l}\text { De novo indications. } \\
\text { Prevention and termination of } \\
\text { atrial tachyarrhythmias does } \\
\text { not represent a stand-alone } \\
\text { indication for pacing }\end{array}$ & III & A & 149-159 \\
\hline
\end{tabular}

${ }^{\mathrm{a}}$ Class of recommendation.

${ }^{\mathrm{b}}$ Level of evidence.

${ }^{c}$ Reference(s) supporting recommendation(s) 


\section{Complications of pacing and CRT implantation}

Pacing and CRT are associated with a substantial rate of complications (Web Table 21). The majority of the information in this field has come from retrospective studies based on implantations performed more than 20 years ago. ${ }^{\text {} 292}$ Recently published

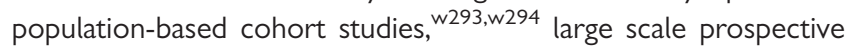
studies and a meta-analysis have provided modern information. ${ }^{57, w 295}$ In a prospective study of first implantation for bradycardia, short- and long-term complications of pacemaker therapy have been reported to be 12.4 and $9.2 \%$, respectively, after careful follow-up. ${ }^{\text {w295 }}$ Overall complication rates increased sharply as indi-

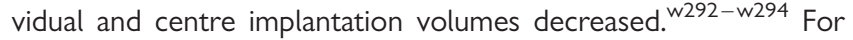
example, in a nationwide registry, the risk of complications was increased by a factor of 1.6 for inexperienced operators with a total of less than 25 implantations. ${ }^{\text {w293 }}$ Lead complications are the main reason for re-operation after implantation of PM or CRT devices. In a nationwide registry of 28860 patients, lead complications occurred in $3.6 \%$ of patients. Complications occurred in $4.3 \%$ of all LV leads, $2.3 \%$ of right atrial leads and $2.2 \%$ of RV leads. The presence of a CRT device (OR 3.3) or a passive-fixation right atrial lead (OR 2.2) were the two most important factors associated with lead complications. $^{\text {w293 }}$

The majority of the complications with pacemakers occur

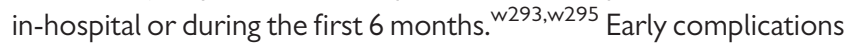
(i.e. occurring after $6-8$ weeks post-implantation) have been reported to range from $5.7 \%$ in a retrospective study to $12.4 \%$ in a recent prospective study of first implantations. ${ }^{\text {w292,w295 }}$ After this period, the complication rate decreases but is still substantial, being reported in $4.8 \%$ of cases at 30 days, $5.5 \%$ at 90 days and $7.5 \%$ at 3 years. $^{\text {w296 }}$ In a recent prospective study of first implantations, higher complication rates were reported in $15.6,18.3$ and $19.7 \%$ of the patients at 1, 3 and 5 years, respectively. ${ }^{\text {w295 }}$

A recent large prospective trial showed that, over 6 months of follow-up, device upgrade or revision is associated with a complication risk ranging from $4 \%$ of patients who had a generator replacement only, to $15.3 \%$ of patients who had a generator replacement or upgrade combined with one or more transvenous lead insertions. $^{\text {w163 }}$ In both cohorts, major complications were higher with CRT-D, compared with PM replacements. Complications were highest in patients who had an upgrade to- or a revised CRT device (18.7\%). These data support careful decision-making before device replacement and when considering upgrades to more complex systems.

A meta-analysis of 9082 patients in 25 CRT trials showed that the implantation success rate was $94.4 \%$; peri-implantation deaths occurred in $0.3 \%$ of trial participants, mechanical complications (including coronary sinus dissection or perforation, pericardial effusion or tamponade, pneumothorax and haemothorax) in 3.2\%, lead problems in $6.2 \%$ and infections in $1.4 \% .{ }^{57}$ Similar rates of complications (totalling 10.5\%) were observed by Reynolds et al. among 7874 Medicare beneficiaries. ${ }^{\text {w297 }}$

Haematomas are very frequent $(2.9-9.5 \%$ of the cases) and are usually managed conservatively. Evacuation is required in $0.3-2 \%$ of implantations and is associated with 15 times the original risk of infection. Many haematomas can be avoided by careful haemostasis and preparation of the patient, allowing correct management of antiplatelet and anticoagulant drugs. Compared with untreated patients, aspirin carries a two-fold risk of bleeding and dual antiplatelet therapy (aspirin plus thienopyridine) carries a four-fold risk of bleeding during the peri-operative period. ${ }^{\text {w298 }}$ The use of clopidogrel or intravenous heparin significantly increases the risk of haematoma at the time of pacemaker implantation. ${ }^{\text {w299 }}$ This risk was reduced by withholding clopidogrel 4 days before implantation. In most cases, antiplatelet medications can safely be discontinued, for a period of 5-7 days, specifically when prescribed for primary prevention of cardiovascular events (Web Table 22). ${ }^{\text {w300,w301 }}$ The use of heparin bridging to oral anticoagulation has also been shown to increase the risk of bleeding and continuation of warfarin (international normalized ratio $<2.0$ ) is proposed by several authors. ${ }^{\mathrm{w} 302-w 304}$ No data are available regarding peri-operative management of patients taking novel oral anticoagulant (thrombin or factor Xa inhibitors). Given the rapid onset and cessation of its action, no bridging therapy with low molecular weight heparin should be required and, following surgery, they should be restarted as soon as effective haemostasis has been achieved. ${ }^{\text {w305 }}$

Infection is one of the most worrying post-operative complications. In a population-based cohort study of 46299 consecutive patients, the incidence of PM infection was 1.82 per 1000 PM-years after the first implantation; the incidence was 4.8 per 1000 PM-years during the first year and 1.0 per 1000 PM-years thereafter. In case of PM replacement these figures increased to 12 per 1000 PM-years and 3.3 per 1000 PM-years, respectively. ${ }^{\text {w306 }}$ In another population-based study, ${ }^{w 07}$ the incidence of definite infection of PM and ICD was 1.9 per 1000 device-years. The incidence of pocket infection without bloodstream infection was 1.37 per 1000 device-years and pocket infection with bloodstream infection or device-related endocarditis was 1.14 per 1000 device-years. Infections occurred more frequently with use of temporary pacing or other procedures before implantation (OR 2.5 and 5.8, respectively), early re-interventions (OR 15) and with lack of antibiotic prophylaxis (OR 2.5). A meta-analysis of antibiotic prophylaxis using a regimen of pre-procedure and post-procedure administration suggested a significant reduction in the incidence of infection. ${ }^{\text {w308 }} \mathrm{A}$ recent large-scale, randomized, double-blind, placebo-controlled trial established the benefit of $1 \mathrm{~g}$ intravenous cefazolin antibiotic, administered immediately before the procedure, in reducing the incidence of procedure-related infection and systemic infections from $3.28 \%$ in patients not receiving antibiotic to $0.63 \%$ in those receiving antibiotic $(P=0.016){ }^{\text {w309 }}$

\section{Management considerations}

\subsection{Pacing from alternative right ventricular sites}

The haemodynamic and clinical effect of pacing from alternative RV sites in the His region, mid- or high ventricular septum and outflow tract has been evaluated in the last two decades (Web Table 23). ${ }^{143, w 310-326}$ In a systematic review and meta-analysis of 14 RCTs for a total of 754 patients, ${ }^{\text {w322 }}$ compared with subjects 
randomized to RV apical pacing, those randomized to $\mathrm{RV}$ non-apical pacing had greater LVEF at the end of follow-up $(+4.27 \%$; $95 \% \mathrm{Cl}$ $1.15-7.40 \%$ ) especially those with a baseline LVEF $<45 \%$ and with a follow-up length $>12$ months. No significant difference was observed in RCTs of patients whose baseline LVEF was preserved. Results were inconclusive with respect to exercise capacity, functional class, quality of life and survival. Results seem to be influenced by the pacing site, with septal pacing being less effective than the outflow tract and para-Hisian region. The complication rate of non- $R V$ apical pacing is similar to that of $R V$ apical pacing. This Task Force is unable to give definite recommendations until the results of larger trials become available.

\subsection{Re-implantation of pacemaker/cardiac resynchronization therapy after device explantation for infection}

Re-implantation is a matter of major concern in patients treated for PM/CRT infection. Although the recent ESC Guidelines on the prevention, diagnosis and treatment of infective endocarditis give some recommendations, ${ }^{\text {w327 }}$ these were based on expert consensus documents, ${ }^{\text {w328,w329 }}$ lacking data derived from randomized trials or large observational registries. In some studies, ${ }^{\text {w330-w332 }}{ }^{2}$ replacement could be avoided in $30-50 \%$ of patients as they lacked strong guideline-based indications for pacing. Thus, the decision to re-implant a device should be weighed carefully.

\section{Location}

The new PM or CRT should be implanted at a different site from the

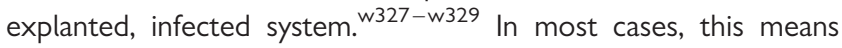
re-implantation on the opposite side of the chest. A new epicardial pacing system can be inserted, particularly if the infected lead is extracted at cardiotomy, or if venous access is impossible.

\section{Timing}

The optimal timing for re-implantation is not known. However, in patients with no evidence of valvular endocarditis or lead vegetations, a new transvenous device can be implanted at a new location after control of local infection, if blood cultures obtained within 24 hours after device removal remain negative for 72 hours. ${ }^{\text {w328,w329 }}$ This early re-implantation approach is usually reserved for patients who are PM-dependent. Indeed, it has recently been shown in a large, single centre, retrospective study that the infection relapse rate was higher in patients implanted during the same hospitalization for hardware removal. ${ }^{\text {w32 }}$ In patients with lead vegetations or valvular endocarditis, a new transvenous implantation should be postponed to 14 days after removal, ${ }^{\text {w328,w329 }}$ or even longer. ${ }^{\text {w327 }}$

\section{Interim management}

Patients who are not PM-dependent can be followed without a temporary PM until a new system is implanted. In patients who are PM-dependent, temporary transvenous pacing is continued until re-implantation. ${ }^{w 327-w 329, w 333}$ Temporary pacing yields the risk of malfunction, introduction of new infections and short duration capability (see section 6.4). The ESC Guidelines on infective endocarditis recommend avoidance of temporary pacing as much as possible. ${ }^{\text {w327 }}$ Alternatively, a new epicardial pacing system can be inserted immediately, particularly if the infected lead is extracted at cardiotomy.

\section{Clinical perspectives:}

- There is general consensus among experts that the indication should be re-assessed before re-implanting a PM after device explantation; if there is an established indication, re-implantation has to be performed at another site.

- For patients who are PM-dependent, the optimal management strategy - i.e. immediate epicardial vs. temporary transvenous pacing — is not well defined.

\subsection{Magnetic resonance imaging in patients with implanted cardiac devices}

Since it is estimated that, after implantation, up to $75 \%$ of patients with PMs develop an indication for magnetic resonance imaging (MRI) examination owing to medical co-morbidities, ${ }^{\text {w334,w335 }}$ this Task Force believes it is necessary to provide recommendations on how to perform an MRI examination safely in patients with conventional devices.

Potential adverse effects of MRI on implanted cardiac devices include: radiofrequency-induced heating of the lead tips, pacing inhibition/dysfunction, asynchronous pacing with the possibility of induction of atrial or ventricular tachyarrhythmias, transient reed switch activation, change or loss of programmed data and changes in capture threshold. ${ }^{\text {w336 }}$ The closer the scanning area is to the system, the higher is the risk. Initial experience at $0.5 \mathrm{~T}$ and subsequently at $1.5 \mathrm{~T}$ suggested that MRI can be performed safely provided that a safety protocol shown below is adopted. ${ }^{160-172, w 337, w 338}$ In the largest study to date, that of Nazarian et al., ${ }^{170}$ which included 438 patients over a period of 7 years, the primary clinically significant event attributable to MRI was the occurrence of power-on-reset events in up to $1.5 \%$ of device recipients. During power-on-reset, the device is susceptible to inhibition of pacing output and activation of anti-tachycardia therapies. Despite this evidence, all ICDs and the overwhelming majority of currently implanted PMs, are considered a contra-indication to MRI by the US Food and Drug Administration and by device manufacturers. $^{\text {w339-w341,w343 }}$

A recent prospective, randomized, controlled, worldwide clinical trial, ${ }^{173}$ which included 258 patients randomized to undergo an MRI over a period of 1.5 years, evaluated the safety and effectiveness of a PM system designed for safe use in MRI for any bradycardia indicated patient. No MRI-related complications occurred during MRI at $1.5 \mathrm{~T}$, nor at 1 week or 1 month postprocedure.

\section{Suggestions for device programming when magnetic resonance imaging is required}

\section{Conventional cardiac devices (Figure 2 and Recommendation 1)}

(i) Because changes in device variables and programming may occur, monitoring by qualified personnel during $\mathrm{MRI}$ is essential. 


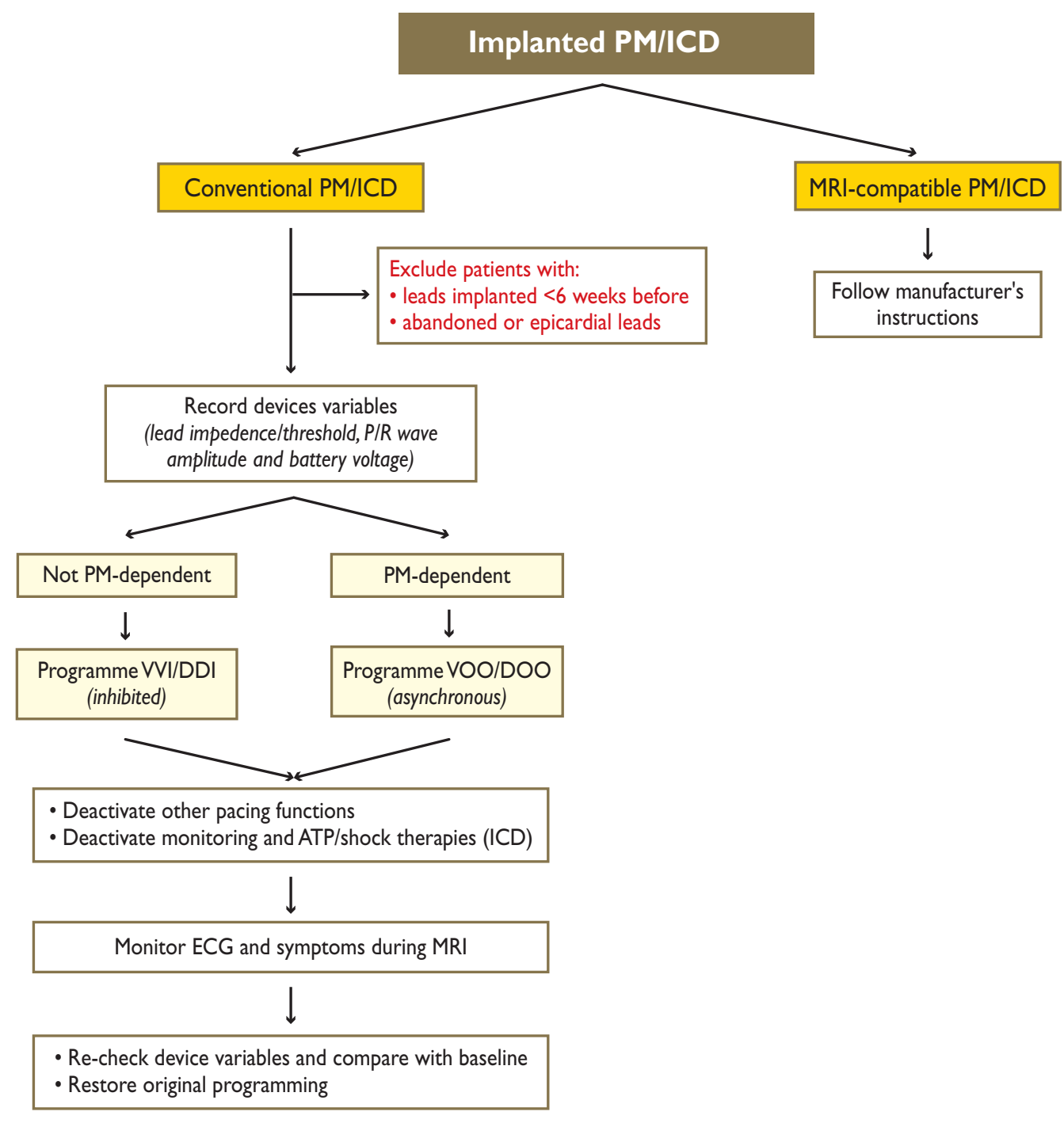

Figure I 2 Safety precautions for magnetic resonance imaging (MRI) in patients with conventional cardiac devices. ATP $=$ anti-tachycardiac pacing; $E C G=$ electrocardiogram; ICD = implantable cardioverter defibrillator; $\mathrm{PM}=$ pacemaker. Adapted from Nazarian et al. ${ }^{170}$

(ii) Exclude patients with leads that have not matured $(<6$ weeks since implantation, during which the leads are prone to spontaneous dislodgement) and those with epicardial and abandoned leads (which are prone to heating).

(iii) Programme an asynchronous pacing mode in PM-dependent patients to avoid inappropriate inhibition of pacing due to detection of electromagnetic interference.

(iv) In contrast, use an inhibited pacing mode for patients without PM dependence, to avoid inappropriate pacing due to tracking of electromagnetic interference.

(v) Deactivate other pacing functions (magnet, rate, noise, PVC, ventricular sense, AF response) in order to ensure that sensing of electromagnetic interference does not lead to unwarranted pacing.

(vi) Deactivate tachyarrhythmia monitoring and therapies (ATP/ shock) to avoid delivery of unwarranted therapies. (vii) Re-programme the device immediately after the MRI examination.

\section{Magnetic resonance imaging-conditional devices (Recommen- dation 2)}

The basic elements are identical to conventional cardiac devices (Figure 12). For MRI-conditional devices, programming as described in (iii), (iv), (v) and (vi) is automatically performed by an external physician-activated device.

\section{Clinical perspectives:}

- When MRI is necessary for clinical management of serious diseases, the benefit of MRI might outweigh the risk of performing MRI. Alternative imaging techniques have to be considered.

- A consultation with the EP specialist is warranted.

- No information exists for MRI performed at >1.5 T, even for MRIcompatible devices. This possibility needs further evaluation. 


\section{Magnetic resonance in patients with implanted cardiac devices}

\begin{tabular}{|l|c|c|c|}
\hline Recommendations & Class $^{\text {a }}$ & Level $^{\text {b }}$ & Ref. $^{\text {c }}$ \\
\hline $\begin{array}{l}\text { I) Conventional cardiac } \\
\text { devices. } \\
\text { In patients with conventional } \\
\text { cardiac devices, MR at I.5 T can } \\
\text { be performed with a low risk } \\
\text { of complications if appropriate } \\
\text { precautions are taken (see } \\
\text { additional advice). }\end{array}$ & Ilb & B & I60-I72 \\
\hline $\begin{array}{l}\text { 2) MR-conditional PM } \\
\text { systems. } \\
\text { In patients with MR-conditional } \\
\text { PM systems, MR at I.5T } \\
\text { can be done safely following } \\
\text { manufacturer instructions. }\end{array}$ & Ila \\
\hline
\end{tabular}

$\mathrm{MRI}=$ magnetic resonance imaging; $\mathrm{PM}=$ pacemaker

${ }^{a}$ Class of recommendation.

'Level of evidence.

'Reference(s) supporting recommendation(s).

\subsection{Emergency (transvenous) temporary pacing}

Complications are common in patients treated with temporary pacing. ${ }^{\text {w34-w351 }}$ These complications are not only restricted to the implantation per se but also involve securing the position of the implanted lead, the change of capture threshold, malfunction, faulty programming or battery depletion of the external PM; it also includes those complications related to the patient, who may extract the pacing lead accidentally. Furthermore, longer use of temporary transvenous pacing may restrict the patient to being bedridden, with accompanying risks for infection and thrombo-embolic events. Without recognition of the potential complications, adverse effects might outweigh the beneficial effects of the PM. Therefore, this Task Force believes that temporary pacing should be avoided as far as possible and, when used, the treatment time should be as brief as possible.

The following issues are relevant as guidance for clinical practice:

- Temporary transvenous pacing shall not be used routinelyand only as a last resort when chronotropic drugs are insufficient.

- Positive chronotropic drug infusion (e.g. isoproterenol, epinephrine, etc.) may be preferred for a limited time, unless there is a contra-indication.

- Temporary transvenous pacing should be limited to cases of (i) high-degree AV block without escape rhythm, (ii) lifethreatening bradyarrhythmias, such as those that occur during interventional procedures (e.g. during percutaneous coronary intervention, etc) or, rarely, in acute settings such as acute myocardial infarction, drug toxicity or concomitant systemic infection.

- If the indications for permanent pacing are established, every effort should be made to implant a permanent PM as soon as possible.

\section{Transcutaneous temporary pacing by an external defibrillator}

This Task Force warns that external pacing provided by patches and an external defibrillator does not provide reliable ventricular stimulation and therefore should only be used, under strict haemodynamic and ECG monitoring, when no other option is available. As soon as possible an alternative action should be undertaken, such as administration of chronotropic drugs or temporary or permanent pacing.

\subsection{Remote management of arrhythmias and device}

The usefulness of remote monitoring has been extensively addressed in the recent joint European and American expert consensus statement on CRT in heart failure to which we refer. ${ }^{\text {w124 }}$ This Task Force endorses that document and believes that there is sufficient evidence to give the recommendation that follows. ${ }^{174-176}$

Diagnosing AF before the first complications occur is a recognized priority for an early starting of anticoagulation therapy and prevention of stroke. Recent data, collected in patients with implanted devices, reinforce the assumption that even short episodes of 'silent' AF convey an increased risk for stroke. ${ }^{153}$ For this issue, we refer to a specific guideline and consensus document. ${ }^{\text {w352,w353 }}$

\begin{tabular}{|c|c|c|c|}
\hline Recommendations & Class $^{a}$ & Level $^{\text {b }}$ & Ref. $^{c}$ \\
\hline $\begin{array}{l}\text { Device-based remote } \\
\text { monitoring should be } \\
\text { considered in order to provide } \\
\text { earlier detection of clinical } \\
\text { problems (e.g. ventricular } \\
\text { tachyarrhythmias, atrial } \\
\text { fibrillation) and technical issues } \\
\text { (e.g. lead fracture, insulation } \\
\text { defect). }\end{array}$ & Ila & $\mathbf{A}$ & $174-176$ \\
\hline
\end{tabular}

${ }^{\mathrm{a}}$ Class of recommendation.

bLevel of evidence.

${ }^{c}$ Reference(s) supporting recommendation(s). 
The CME text '2013 ESC Guidelines on cardiac pacing and cardiac resynchronization therapy' is accredited by the European Board for Accreditation in Cardiology (EBAC). EBAC works according to the quality standards of the European Accreditation Council for Continuing Medical Education (EACCME), which is an institution of the European Union of Medical Specialists (UEMS). In compliance with EBAC/EACCME Guidelines, all authors participating in this programme have disclosed any potential conflicts of interest that might cause a bias in the article. The Organizing Committee is responsible for ensuring that all potential conflicts of interest relevant to the programme are declared to the participants prior to the CME activities.

CME questions for this article are available at: European Heart Journal http://www.oxforde-learning.com/eurheartj and European Society of Cardiology http://www.escardio. org/guidelines.

\section{References}

Addenda: Additional references are mentioned with ' $w$ ' in the main text and can be found on the online addenda along with 5 figures $(1,6,7,9,11,12)$ and 10 tables $(3,4,5,9,11,12,19,21,22,23)$. They are available on the ESC website only at http:// www.escardio.org/guidelines-surveys/esc-guidelines/Pages/cardiac-pacing-and-cardiacresynchronisation-therapy.aspx

1. Alboni P, Menozzi C, Brignole M, Paparella N, Gaggioli G, Lolli G, Cappato R. Effects of permanent pacemaker and oral theophylline in sick sinus syndrome the THEOPACE study: a randomized controlled trial. Circulation 1997;96:260-266.

2. Healey JS, ToffWD, Lamas GA, Andersen HR, Thorpe KE, Ellenbogen KA, Lee KL, Skene AM, Schron EB, Skehan JD, Goldman L, Roberts RS, Camm AJ, Yusuf S, Connolly SJ. Cardiovascular outcomes with atrial-based pacing compared with ventricular pacing: meta-analysis of randomized trials, using individual patient data. Circulation 2006;114:11-17.

3. Nielsen JC, Thomsen PE, Hojberg S, Moller M, Vesterlund T, Dalsgaard D, Mortensen LS, Nielsen T, Asklund M, Friis EV, Christensen PD, Simonsen EH, Eriksen UH, Jensen GV, Svendsen JH, Toff WD, Healey JS, Andersen HR. A comparison of single-lead atrial pacing with dual-chamber pacing in sick sinus syndrome. Eur Heart J 2011;32:686-696.

4. Brignole M, DeharoJC, De Roy L, Menozzi C, Blommaert D, Dabiri L, RufJ, Guieu R. Syncope due to idiopathic paroxysmal atrioventricular block: long-term follow-up of a distinct form of atrioventricular block. J Am Coll Cardiol 2011;58:167-173.

5. Brignole M, Sutton R, Menozzi C, Garcia-Civera R, Moya A, Wieling W, Andresen D, Benditt DG, Vardas P. Early application of an implantable loop recorder allows effective specific therapy in patients with recurrent suspected neurally mediated syncope. Eur Heart J 2006;27:1085-1092.

6. Breivik K, Ohm OJ, Segadal L. Sick sinus syndrome treated with permanent pacemaker in 109 patients. A follow-up study. Acta Med Scand 1979;206:153-159.

7. Hartel G, Talvensaari T. Treatment of sinoatrial syndrome with permanent cardiac pacing in 90 patients. Acta Med Scand 1975;198:341-347.

8. Rasmussen K. Chronic sinus node disease: natural course and indications for pacing. Eur Heart J 1981;2:455-459.

9. Sasaki Y, Shimotori M, Akahane K, Yonekura H, Hirano K, Endoh R, Koike S, Kawa S, Furuta S, Homma T. Long-term follow-up of patients with sick sinus syndrome: a comparison of clinical aspects among unpaced, ventricular inhibited paced, and physiologically paced groups. Pacing Clin Electrophysiol 1988;11:1575-1583.

10. Andersen HR, Thuesen L, Bagger JP, Vesterlund T, Thomsen PE. Prospective randomised trial of atrial versus ventricular pacing in sick-sinus syndrome. Lancet 1994;344:1523-1528.

11. Connolly SJ, Kerr CR, Gent M, Roberts RS, YusufS, Gillis AM, Sami MH, Talajic M, Tang AS, Klein GJ, Lau C, Newman DM. Effects of physiologic pacing versus ventricular pacing on the risk of stroke and death due to cardiovascular causes. Canadian Trial of Physiologic Pacing Investigators. N Engl J Med 2000;342:1385-1391.

12. Lamas GA, Lee KL, Sweeney MO, Silverman R, Leon A, Yee R, Marinchak RA, Flaker G, Schron E, Orav EJ, Hellkamp AS, Greer S, McAnulty J, Ellenbogen K, Ehlert F, Freedman RA, Estes NA 3rd, Greenspon A, Goldman L. Ventricular pacing or dual-chamber pacing for sinus-node dysfunction. N Engl J Med 2002; 346:1854-1862.

13. Lamas GA, Orav EJ, Stambler BS, Ellenbogen KA, Sgarbossa EB, Huang SK, Marinchak RA, Estes NA 3rd, Mitchell GF, Lieberman EH, Mangione CM, Goldman L. Quality of life and clinical outcomes in elderly patients treated with ventricular pacing as compared with dual-chamber pacing. Pacemaker Selection in the Elderly Investigators. N Engl J Med 1998;338:1097-1104.

14. Toff WD, Camm AJ, Skehan JD. Single-chamber versus dual-chamber pacing for high-grade atrioventricular block. N Engl J Med 2005;353:145-155.

15. Castelnuovo E, Stein K, Pitt M, Garside R, Payne E. The effectiveness and costeffectiveness of dual-chamber pacemakers compared with single-chamber pacemakers for bradycardia due to atrioventricular block or sick sinus syndrome: systematic review and economic evaluation. Health Technol Assess 2005;9:iii, xi-xiii, $1-246$.

16. Sweeney MO, Bank AJ, Nsah E, Koullick M, Zeng QC, Hettrick D, Sheldon T, Lamas GA. Minimizing ventricular pacing to reduce atrial fibrillation in sinus-node disease. N Engl J Med 2007;357:1000-1008.
17. Wilkoff BL, Cook JR, Epstein AE, Greene HL, Hallstrom AP, Hsia H, Kutalek SP, Sharma A. Dual-chamber pacing or ventricular backup pacing in patients with an implantable defibrillator: the Dual Chamber and VVI Implantable Defibrillator (DAVID) Trial. JAMA 2002;288:3115-3123.

18. Sud S, Klein GJ, Skanes AC, Gula LJ, Yee R, Krahn AD. Implications of mechanism of bradycardia on response to pacing in patients with unexplained syncope. Europace 2007;9:312-318.

19. Brignole M, Menozzi C, Moya A, Andresen D, Blanc JJ, Krahn AD, Wieling W, Beiras X, Deharo JC, Russo V, Tomaino M, Sutton R. Pacemaker therapy in patients with neurally mediated syncope and documented asystole: Third International Study on Syncope of Uncertain Etiology (ISSUE-3): a randomized trial. Circulation 2012;125:2566-2571.

20. Ammirati F, Colivicchi F, Santini M. Permanent cardiac pacing versus medical treatment for the prevention of recurrent vasovagal syncope: a multicenter, randomized, controlled trial. Circulation 2001;104:52-57.

21. Connolly SJ, Sheldon R, Roberts RS, Gent M. The North American Vasovagal Pacemaker Study (VPS). A randomized trial of permanent cardiac pacing for the prevention of vasovagal syncope. J Am Coll Cardiol 1999;33:16-20.

22. Connolly SJ, Sheldon R, Thorpe KE, Roberts RS, Ellenbogen KA, Wilkoff BL, Morillo C, Gent M. Pacemaker therapy for prevention of syncope in patients with recurrent severe vasovagal syncope: Second Vasovagal Pacemaker Study (VPS II): a randomized trial. JAMA 2003;289:2224-2229.

23. Raviele A, Giada F, Menozzi C, Speca G, Orazi S, Gasparini G, Sutton R, Brignole M. A randomized, double-blind, placebo-controlled study of permanent cardiac pacing for the treatment of recurrent tilt-induced vasovagal syncope. The vasovagal syncope and pacing trial (SYNPACE). Eur Heart J 2004;25:1741-1748.

24. Sutton R, Brignole M, Menozzi C, Raviele A, Alboni P, Giani P, Moya A. Dualchamber pacing in the treatment of neurally mediated tilt-positive cardio-inhibitory syncope: pacemaker versus no therapy: a multicenter randomized study. The Vasovagal Syncope International Study (VASIS) Investigators. Circulation 2000;102: 294-299.

25. Moya A, Garcia-Civera R, Croci F, Menozzi C, Brugada J, Ammirati F, Del Rosso A, Bellver-Navarro A, Garcia-Sacristan J, Bortnik M, Mont L, Ruiz-Granell R, Navarro $X$. Diagnosis, management, and outcomes of patients with syncope and bundle branch block. Eur Heart J 2011;32:1535-1541.

26. Scheinman MM, Peters RW, Suave MJ, Desai J, Abbott JA, Cogan J, Wohl B, Williams K. Value of the H-Q interval in patients with bundle branch block and the role of prophylactic permanent pacing. Am J Cardiol 1982;50:1316-1322.

27. Gronda M, Magnani A, Occhetta E, Sauro G, D’Aulerio M, Carfora A, Rossi P. Electrophysiological study of atrio-ventricular block and ventricular conduction defects. Prognostic and therapeutical implications. G Ital Cardiol 1984;14:768-773.

28. Bergfeldt L, Edvardsson N, Rosenqvist M, Vallin H, Edhag O. Atrioventricular block progression in patients with bifascicular block assessed by repeated electrocardiography and a bradycardia-detecting pacemaker. Am J Cardiol 1994;74:1129-1132.

29. Dini $P$, laolongo D, Adinolfi E et al. Prognostic value of His-ventricular conduction after ajmaline administration. In: Masoni A, Albani P, (eds). Cardiac Electrophysiology Today. London: Academic Press; 1982, 515-522.

30. Kaul U, Dev V, Narula J, Malhotra AK, Talwar KK, Bhatia ML. Evaluation of patients with bundle branch block and 'unexplained' syncope: a study based on comprehensive electrophysiological testing and ajmaline stress. Pacing Clin Electrophysiol 1988; 11:289-297.

31. Twidale N, Heddle WF, Tonkin AM. Procainamide administration during electrophysiology study-utility as a provocative test for intermittent atrioventricular block. Pacing Clin Electrophysiol 1988;11:1388-1397.

32. Santini M, Castro A, Giada F, Ricci R, Inama G, Gaggioli G, Calò L, Orazi S, Viscusi M, Chiodi L, Bartoletti A, Foglia-Manzillo G, Ammirati F, Loricchio ML, Pedrinazzi C, Turreni F, Gasparini G, Accardi F, Raciti G, Raviele A. Prevention of syncope through permanent cardiac pacing in patients with bifascicular block and syncope of unexplained origin: the PRESS study. Circ Arrhythm Electrophysiol. 2013;6: 101-107.

33. McAnulty JH, Rahimtoola SH, Murphy E, DeMots H, Ritzmann L, Kanarek PE, Kauffman S. Natural history of 'high-risk' bundle-branch block: final report of a prospective study. N Engl J Med 1982;307:137-143.

34. Peters RW, Scheinman MM, Modin C, O'Young J, Somelofski CA, Mies C. Prophylactic permanent pacemakers for patients with chronic bundle branch block. Am J Med 1979;66:978-985. 
35. Brignole M, Menozzi C. The natural history of carotid sinus syncope and the effect of cardiac pacing. Europace 2011;13:462-464.

36. Brignole M, Menozzi C, Lolli G, Bottoni N, Gaggioli G. Long-term outcome of paced and nonpaced patients with severe carotid sinus syndrome. Am J Cardiol 1992;69: 1039-1043.

37. Claesson JE, Kristensson BE, Edvardsson N, Wahrborg P. Less syncope and milder symptoms in patients treated with pacing for induced cardio-inhibitory carotid sinus syndrome: a randomized study. Europace 2007;9:932-936.

38. Sugrue DD, Gersh BJ, Holmes DR Jr., Wood DL, Osborn MJ, Hammill SC. Symptomatic 'isolated' carotid sinus hypersensitivity: natural history and results of treatment with anticholinergic drugs or pacemaker. J Am Coll Cardiol 1986;7:158-162.

39. Gaggioli G, Brignole M, Menozzi C, Devoto G, Oddone D, Gianfranchi L, Gostoli E, Bottoni N, Lolli G. A positive response to head-up tilt testing predicts syncopal recurrence in carotid sinus syndrome patients with permanent pacemakers. Am J Cardiol 1995;76:720-722.

40. Maggi R, Menozzi C, Brignole M, Podoleanu C, lori M, Sutton R, Moya A, Giada F, Orazi S, Grovale N. Cardio-inhibitory carotid sinus hypersensitivity predicts an asystolic mechanism of spontaneous neurally mediated syncope. Europace 2007; 9:563-567.

41. Madigan NP, Flaker GC, Curtis JJ, Reid J, Mueller KJ, Murphy TJ. Carotid sinus hypersensitivity: beneficial effects of dual-chamber pacing. Am J Cardiol 1984;53: 1034-1040.

42. Brignole M, Sartore B, Barra M, Menozzi C, Lolli G. Is DDD superior to VVI pacing in mixed carotid sinus syndrome? An acute and medium-term study. Pacing Clin Electrophysiol 1988;11:1902-1910.

43. Sutton R. Pacing in patients with carotid sinus and vasovagal syndromes. Pacing Clin Electrophysiol 1989;12:1260-1263.

44. Flammang D, Church TR, De Roy L, Blanc J), Leroy J, Mairesse GH, Otmani A, Graux PJ, Frank R, Purnode P. Treatment of unexplained syncope: a multicenter, randomized trial of cardiac pacing guided by adenosine 5'-triphosphate testing. Circulation 2012;125:31-36.

45. Parry SW, Steen N, Bexton RS, Tynan M, Kenny RA. Pacing in elderly recurrent fallers with carotid sinus hypersensitivity: a randomised, double-blind, placebo controlled crossover trial. Heart 2009;95:405-409.

46. Cleland JG, Freemantle N, Erdmann E, Gras D, Kappenberger L, Tavazzi L, Daubert JC. Long-term mortality with cardiac resynchronization therapy in the Cardiac Resynchronization-Heart Failure (CARE-HF) trial. Eur J Heart Fail 2012; 14:628-634.

47. Bogale N, Witte K, Priori S, Cleland J, Auricchio A, Gadler F, Gitt A, Limbourg T, Linde C, Dickstein K. The European Cardiac Resynchronization Therapy Survey: comparison of outcomes between de novo cardiac resynchronization therapy implantations and upgrades. Eur J Heart Fail 2011;13:974-983.

48. Zareba W, Klein H, Cygankiewicz I, Hall WJ, McNitt S, Brown M, Cannom D, Daubert JP, Eldar M, Gold MR, Goldberger JJ, Goldenberg I, Lichstein E, Pitschner H, Rashtian M, Solomon S, Viskin S, Wang P, Moss AJ. Effectiveness of Cardiac Resynchronization Therapy by QRS Morphology in the Multicenter Automatic Defibrillator Implantation Trial-Cardiac Resynchronization Therapy (MADIT-CRT). Circulation 2011;123:1061-1072.

49. Abraham WT, Fisher WG, Smith AL, Delurgio DB, Leon AR, Loh E, Kocovic DZ, Packer M, Clavell AL, Hayes DL, Ellestad M, Trupp RJ, Underwood J, Pickering F, Truex C, McAtee P, Messenger J. Cardiac resynchronization in chronic heart failure. N Engl J Med 2002;346:1845-1853.

50. Moss AJ, Hall WJ, Cannom DS, Klein H, Brown MW, Daubert JP, Estes NA 3rd, Foster E, Greenberg H, Higgins SL, Pfeffer MA, Solomon SD, Wilber D, Zareba W. Cardiac-resynchronization therapy for the prevention of heart-failure events. N Engl J Med 2009;361:1329-1338.

51. Auricchio A, Stellbrink C, Butter C, Sack S, Vogt J, Misier AR, Bocker D, Block M, Kirkels JH, Kramer A, Huvelle E. Clinical efficacy of cardiac resynchronization therapy using left ventricular pacing in heart failure patients stratified by severity of ventricular conduction delay. J Am Coll Cardiol 2003;42:2109-2116.

52. Cazeau S, Leclercq C, Lavergne T, Walker S, Varma C, Linde C, Garrigue S, Kappenberger L, Haywood GA, Santini M, Bailleul C, Daubert JC. Effects of multisite biventricular pacing in patients with heart failure and intraventricular conduction delay. N Engl J Med 2001;344:873-880.

53. Higgins SL, Hummel JD, Niazi IK, Giudici MC, Worley SJ, Saxon LA, Boehmer JP, Higginbotham MB, De Marco T, Foster E, Yong PG. Cardiac resynchronization therapy for the treatment of heart failure in patients with intraventricular conduction delay and malignant ventricular tachyarrhythmias. J Am Coll Cardiol 2003;42: 1454-1459.

54. Young JB, Abraham WT, Smith AL, Leon AR, Lieberman R, Wilkoff B, Canby RC, Schroeder JS, Liem LB, Hall S, Wheelan K. Combined cardiac resynchronization and implantable cardioversion defibrillation in advanced chronic heart failure: the MIRACLE ICD Trial. JAMA 2003;289:2685-2694.

55. Bristow MR, Saxon LA, Boehmer J, Krueger S, Kass DA, De Marco T, Carson P, DiCarlo L, DeMets D, White BG, DeVries DW, Feldman AM.
Cardiac-resynchronization therapy with or without an implantable defibrillator in advanced chronic heart failure. N Engl J Med 2004;350:2140-2150.

56. Cleland JG, Daubert JC, Erdmann E, Freemantle N, Gras D, Kappenberger L, Tavazzi $L$. The effect of cardiac resynchronization on morbidity and mortality in heart failure. N EnglJ Med 2005;352:1539-1549.

57. Al-Majed NS, McAlister FA, Bakal JA, Ezekowitz JA. Meta-analysis: cardiac resynchronization therapy for patients with less symptomatic heart failure. Ann Intern Med 2011;154:401-412.

58. Sipahi I, Carrigan TP, Rowland DY, Stambler BS, FangJC. Impact of QRS duration on clinical event reduction with cardiac resynchronization therapy: meta-analysis of randomized controlled trials. Arch Intern Med 2011;171:1454-1462.

59. Gervais R, Leclercq C, Shankar A, Jacobs S, Eiskjaer H, Johannessen A, Freemantle N, Cleland JG, Tavazzi L, Daubert C. Surface electrocardiogram to predict outcome in candidates for cardiac resynchronization therapy: a sub-analysis of the CARE-HF trial. Eur J Heart Fail 2009;11:699-705.

60. Abraham WT, Young JB, Leon AR, Adler S, BankAJ, Hall SA, Lieberman R, Liem LB, O'Connell JB, Schroeder JS, Wheelan KR. Effects of cardiac resynchronization on disease progression in patients with left ventricular systolic dysfunction, an indication for an implantable cardioverter-defibrillator, and mildly symptomatic chronic heart failure. Circulation 2004;110:2864-2868.

61. Linde C, Abraham WT, Gold MR, St John Sutton M, Ghio S, Daubert C. Randomized trial of cardiac resynchronization in mildly symptomatic heart failure patients and in asymptomatic patients with left ventricular dysfunction and previous heart failure symptoms. J Am Coll Cardiol 2008;52:1834-1843.

62. Tang AS, Wells GA, Talajic M, Arnold MO, Sheldon R, Connolly S, Hohnloser SH, Nichol G, Birnie DH, Sapp JL, Yee R, Healey JS, Rouleau JL. Cardiac-resynchronization therapy for mild-to-moderate heart failure. $N$ Engl J Med 2010;363:2385-2395.

63. Gold MR, Thebault C, Linde C, Abraham WT, Gerritse B, Ghio S, St John Sutton M, Daubert JC. Effect of QRS duration and morphology on cardiac resynchronization therapy outcomes in mild heart failure: results from the Resynchronization Reverses Remodelling in Systolic Left Ventricular Dysfunction (REVERSE) study. Circulation 2012;126:822-829.

64. Sipahi I, Chou JC, Hyden M, Rowland DY, Simon DI, Fang JC. Effect of QRS morphology on clinical event reduction with cardiac resynchronization therapy: meta-analysis of randomized controlled trials. Am Heart /2012;163:260-267 e263.

65. Beshai JF, Grimm RA, Nagueh SF, Baker JH 2nd, Beau SL, Greenberg SM, Pires LA, Tchou PJ. Cardiac-resynchronization therapy in heart failure with narrow QRS complexes. N Engl J Med 2007;357:2461-2471.

66. Thibault B, Harel F, Ducharme A, White M, Ellenbogen KA, Frasure-Smith N Roy D, Philippon F, Dorian P, Talajic M, Dubuc M, Guerra PG, Macle L, Rivard L, Andrade J, Khairy P, LESSER-EARTH Investigators. Cardiac Resynchronization Therapy in Patients With Heart Failure and a QRS Complex $<120$ Milliseconds: The Evaluation of Resynchronization Therapy for Heart Failure (LESSER-EARTH) Trial. Circulation 2013;127:873-881.

67. Koplan BA, Kaplan AJ, Weiner S, Jones PW, Seth M, Christman SA. Heart failure decompensation and all-cause mortality in relation to percent biventricular pacing in patients with heart failure: is a goal of $100 \%$ biventricular pacing necessary? J Am Coll Cardiol 2009;53:355-360.

68. Hayes DL, Boehmer JP, Day JD, Gilliam FR 3rd, Heidenreich PA, Seth M, Jones PW Saxon LA. Cardiac resynchronization therapy and the relationship of percent biventricular pacing to symptoms and survival. Heart Rhythm 2011;8:1469-1475.

69. Cheng A, Landman SR, Stadler RW. Reasons for loss of cardiac resynchronization therapy pacing: insights from 32844 patients. Circ Arrhythm Electrophysiol 2012;5: 884-888

70. Saxon LA, Olshansky B, Volosin K, Steinberg JS, Lee BK, Tomassoni G, Guarnieri T, Rao A, Yong P, Galle E, Leigh J, Ecklund F, Bristow MR. Influence of left ventricular lead location on outcomes in the COMPANION study. J Cardiovasc Electrophysiol 2009;20:764-768

71. Thebault C, Donal E, Meunier C, Gervais R, Gerritse B, Gold MR, Abraham WT, Linde C, Daubert JC. Sites of left and right ventricular lead implantation and response to cardiac resynchronization therapy observations from the REVERSE trial. Eur Heart J 2012;33:2662-2671.

72. Singh JP, Klein HU, Huang DT, Reek S, Kuniss M, Quesada A, Barsheshet A, Cannom D, Goldenberg I, McNitt S, Daubert JP, Zareba W, Moss AJ. Left ventricular lead position and clinical outcome in the multicenter automatic defibrillator implantation trial-cardiac resynchronization therapy (MADIT-CRT) trial. Circulation 2011:123:1159-1166.

73. Khan FZ, Virdee MS, Palmer CR, Pugh PJ, O'Halloran D, Elsik M, Read PA, Begley D Fynn SP, Dutka DP. Targeted left ventricular lead placement to guide cardiac resynchronization therapy: the TARGET study: a randomized, controlled trial. J Am Coll Cardiol 2012;59:1509-1518.

74. Sawhney NS, Waggoner AD, Garhwal S, Chawla MK, Osborn J, Faddis MN. Randomized prospective trial of atrioventricular delay programming for cardiac resynchronization therapy. Heart Rhythm 2004;1:562-567. 
75. Vidal B, Tamborero D, Mont L, Sitges M, Delgado V, Berruezo A, Diaz-Infante E, Tolosana JM, Pare C, Brugada J. Electrocardiographic optimization of interventricular delay in cardiac resynchronization therapy: a simple method to optimize the device. J Cardiovasc Electrophysiol 2007;18:1252-1257.

76. Abraham WT, Calò L, Islam N, Klein N, Alawwa A, Exner D, Goodman J, Messano L, Clyne C, Pelargonio G, Hasan A, Seidl K, Sheppard R, Yu CM, Herre J, Lee LY, Boulogne E, Petrutiu S, Birgersdotter-Green U, Gras D. Randomized controlled trial of frequent optimization of cardiac resynchronization therapy: results of the Frequent Optimization Study Using the QuickOpt ${ }^{\text {TM }}$ Method (FREEDOM) Trial. (in press).

77. Abraham WT, Leon AR, St John Sutton MG, Keteyian SJ, Fieberg AM, Chinchoy E, Haas $G$. Randomized controlled trial comparing simultaneous versus optimized sequential interventricular stimulation during cardiac resynchronization therapy. Am Heart / 2012;164:735-741.

78. Boriani G, Muller CP, Seidl KH, Grove R, Vogt J, Danschel W, Schuchert A, Djiane P, Biffi M, Becker T, Bailleul C, Trappe HJ. Randomized comparison of simultaneous biventricular stimulation versus optimized interventricular delay in cardiac resynchronization therapy. The Resynchronization for the HemodYnamic Treatment for Heart Failure Management II implantable cardioverter defibrillator (RHYTHM II ICD) study. Am Heart J 2006;151:1050-1058.

79. Ellenbogen KA, Gold MR, Meyer TE, Fernndez Lozano I, Mittal S, Waggoner AD, Lemke B, Singh JP, Spinale FG, Van Eyk JE, Whitehill J, Weiner S, Bedi M, Rapkin J, Stein KM. Primary results from the SmartDelay determined AV optimization: a comparison with other AV delay methods used in cardiac resynchronization therapy (SMART-AV) trial: a randomized trial comparing empirical, echocardiography-guided, and algorithmic atrioventricular delay programming in cardiac resynchronization therapy. Circulation 2010;122:2660-2668.

80. Leon AR, Abraham WT, Brozena S, Daubert JP, Fisher WG, Gurley JC, Liang CS, Wong $G$. Cardiac resynchronization with sequential biventricular pacing for the treatment of moderate-to-severe heart failure. J Am Coll Cardiol 2005;46: 2298-2304.

81. Martin DO, Lemke B, Birnie D, Krum H, Lee KL, Aonuma K, Gasparini M, Starling RC, Milasinovic G, Rogers T, Sambelashvili A, Gorcsan J 3rd, Houmsse M. Investigation of a novel algorithm for synchronized left-ventricular pacing and ambulatory optimization of cardiac resynchronization therapy: results of the adaptive CRT trial. Heart Rhythm 2012;9:1807-1814.

82. Rao RK, Kumar UN, Schafer J, Viloria E, De Lurgio D, Foster E. Reduced ventricular volumes and improved systolic function with cardiac resynchronization therapy: a randomized trial comparing simultaneous biventricular pacing, sequential biventricular pacing, and left ventricular pacing. Circulation 2007;115:2136-2144.

83. Ritter P, Delnoy PP, Padeletti L, Lunati M, Naegele H, Borri-Brunetto A, SilvestreJ. A randomized pilot study of optimization of cardiac resynchronization therapy in sinus rhythm patients using a peak endocardial acceleration sensor vs. standard methods. Europace 2012;14:1324-1333.

84. Gasparini M, Bocchiardo M, Lunati M, Ravazzi PA, Santini M, Zardini M, Signorelli S, Passardi M, Klersy C. Comparison of 1-year effects of left ventricular and biventricular pacing in patients with heart failure who have ventricular arrhythmias and left bundle-branch block: the Bi vs. Left Ventricular Pacing: an International Pilot Evaluation on Heart Failure Patients with Ventricular Arrhythmias (BELIEVE) multicenter prospective randomized pilot study. Am Heart J 2006;152:155 e151-e157.

85. Boriani G, Kranig W, Donal E, Calo L, Casella M, Delarche N, Lozano IF, Ansalone G, Biffi M, Boulogne E, Leclercq C. A randomized double-blind comparison of biventricular versus left ventricular stimulation for cardiac resynchronization therapy: the Biventricular versus Left Univentricular Pacing with ICD Back-up in Heart Failure Patients (B-LEFT HF) trial. Am Heart J 2010;159:1052-1058 e1051.

86. Liang Y, Pan W, Su Y, Ge J. Meta-analysis of randomized controlled trials comparing isolated left ventricular and biventricular pacing in patients with chronic heart failure. Am J Cardiol 2011;108:1160-1165.

87. Thibault B, Ducharme A, Harel F, White M, O'Meara E, Guertin MC, Lavoie J, Frasure-Smith N, Dubuc M, Guerra P, Macle L, Rivard L, Roy D, Talajic M, Khairy $P$. Left ventricular versus simultaneous biventricular pacing in patients with heart failure and a QRS complex $>/=120$ milliseconds. Circulation 2011; 124:2874-2881.

88. Boriani G, Gardini B, Diemberger I, Bacchi Reggiani ML, Biffi M, Martignani C, Ziacchi M, Valzania C, Gasparini M, Padeletti L, Branzi A. Meta-analysis of randomized controlled trials evaluating left ventricular vs. biventricular pacing in heart failure: effect on all-cause mortality and hospitalizations. Eur J Heart Fail 2012;14: $652-660$.

89. Brignole M, Botto G, Mont L, lacopino S, De Marchi G, Oddone D, Luzi M, Tolosana JM, Navazio A, Menozzi C. Cardiac resynchronization therapy in patients undergoing atrioventricular junction ablation for permanent atrial fibrillation: a randomized trial. Eur Heart J 2011;32:2420-2429.

90. Leclercq C, Walker S, Linde C, Clementy J, Marshall AJ, Ritter P, Djiane P, Mabo P, Levy T, Gadler F, Bailleul C, Daubert JC. Comparative effects of permanent biventricular and right-univentricular pacing in heart failure patients with chronic atrial fibrillation. Eur Heart J 2002;23:1780-1787.

91. Upadhyay GA, Choudhry NK, Auricchio A, Ruskin J, Singh JP. Cardiac resynchronization in patients with atrial fibrillation: a meta-analysis of prospective cohort studies. J Am Coll Cardiol 2008;52:1239-1246.

92. Wilton SB, Leung AA, Ghali WA, Faris P, Exner DV. Outcomes of cardiac resynchronization therapy in patients with versus those without atrial fibrillation: a systematic review and meta-analysis. Heart Rhythm 2011;8:1088-1094.

93. Linde C, Leclercq C, Rex S, Garrigue S, Lavergne T, Cazeau S, McKenna W, Fitzgerald M, Deharo JC, Alonso C, Walker S, Braunschweig F, Bailleul C, Daubert JC. Long-term benefits of biventricular pacing in congestive heart failure: results from the MUltisite STimulation in cardiomyopathy (MUSTIC) study. J Am Coll Cardiol 2002;40:111-118.

94. Doshi RN, Daoud EG, Fellows C, Turk K, Duran A, Hamdan MH, Pires LA. Left ventricular-based cardiac stimulation post AV nodal ablation evaluation (the PAVE study). J Cardiovasc Electrophysiol 2005;16:1160-1165.

95. Wein S, Voskoboinik A, Wein L, Billah B, Krum H. Extending the boundaries of cardiac resynchronization therapy: efficacy in atrial fibrillation, New York heart association class II, and narrow QRS heart failure patients. J Card Fail 2010;16: $432-438$.

96. Kamath GS, Cotiga D, Koneru JN, Arshad A, Pierce W, Aziz EF, Mandava A, Mittal S, Steinberg JS. The utility of 12-lead Holter monitoring in patients with permanent atrial fibrillation for the identification of nonresponders after cardiac resynchronization therapy. J Am Coll Cardiol 2009;53:1050-1055.

97. Tolosana JM, Hernandez Madrid A, Brugada J, Sitges M, Garcia Bolao I, Fernandez Lozano I, Martinez Ferrer J, Quesada A, Macias A, Marin W, Escudier JM Gomez AA, Gimenez Alcala M, Tamborero D, Berruezo A, Mont L. Comparison of benefits and mortality in cardiac resynchronization therapy in patients with atrial fibrillation versus patients in sinus rhythm (Results of the Spanish Atrial Fibrillation and Resynchronization [SPARE] Study). Am J Cardiol 2008;102:444-449.

98. Ferreira AM, Adragao P, Cavaco DM, Candeias R, Morgado FB, Santos KR, Santos E, Silva JA. Benefit of cardiac resynchronization therapy in atrial fibrillation patients vs. patients in sinus rhythm: the role of atrioventricular junction ablation. Europace 2008;10:809-815.

99. Ganesan AN, Brooks AG, Roberts-Thomson KC, Lau DH, Kalman JM, Sanders P. Role of $A V$ nodal ablation in cardiac resynchronization in patients with coexistent atrial fibrillation and heart failure a systematic review. J Am Coll Cardiol 2012;59. 719-726.

100. Gasparini M, Auricchio A, Metra M, Regoli F, Fantoni C, Lamp B, Curnis A, Vogt J, Klersy $C$. Long-term survival in patients undergoing cardiac resynchronization therapy: the importance of performing atrio-ventricular junction ablation in patients with permanent atrial fibrillation. Eur Heart J 2008;29:1644-1652.

101. Gasparini M, Auricchio A, Regoli F, Fantoni C, Kawabata M, Galimberti P, Pini D, Ceriotti C, Gronda E, Klersy C, Fratini S, Klein HH. Four-year efficacy of cardiac resynchronization therapy on exercise tolerance and disease progression: the importance of performing atrioventricular junction ablation in patients with atrial fibrillation. J Am Coll Cardiol 2006;48:734-743.

102. Molhoek SG, Bax JJ, Bleeker GB, Boersma E, van Erven L, Steendijk P, van der Wall EE, Schalij MJ. Comparison of response to cardiac resynchronization therapy in patients with sinus rhythm versus chronic atrial fibrillation. Am J Cardiol 2004:94:1506-1509.

103. Delnoy PP, Ottervanger JP, Luttikhuis HO, Elvan A, Misier AR, Beukema WP, van Hemel NM. Comparison of usefulness of cardiac resynchronization therapy in patients with atrial fibrillation and heart failure versus patients with sinus rhythm and heart failure. Am J Cardiol 2007;99:1252-1257.

104. Khadjooi K, Foley PW, Chalil S, Anthony J, Smith RE, Frenneaux MP, Leyva F. Longterm effects of cardiac resynchronisation therapy in patients with atrial fibrillation. Heart 2008;94:879-883.

105. Orlov MV, Gardin JM, Slawsky M, Bess RL, Cohen G, Bailey W, Plumb V, Flathmann H, de Metz K. Biventricular pacing improves cardiac function and prevents further left atrial remodelling in patients with symptomatic atrial fibrillation after atrioventricular node ablation. Am Heart J 2010;159:264-270.

106. Brignole M, Gammage M, Puggioni E, Alboni $P$, Raviele A, Sutton R, Vardas $P$, Bongiorni MG, Bergfeldt L, Menozzi C, Musso G. Comparative assessment of right, left, and biventricular pacing in patients with permanent atrial fibrillation. Eur Heart J 2005;26:712-722.

107. Brignole M, Botto GL, Mont L, Oddone D, lacopino S, De Marchi G, Campoli M, Sebastiani V, Vincenti A, Garcia Medina D, Osca Asensi J, Mocini A, Grovale N, De Santo T, Menozzi C. Predictors of clinical efficacy of 'Ablate and Pace' therapy in patients with permanent atrial fibrillation. Heart 2012;98:297-302.

108. Leclercq C, Cazeau S, Lellouche D, Fossati F, Anselme F, Davy JM, Sadoul N, Klug D, Mollo L, Daubert JC. Upgrading from single chamber right ventricular to biventricular pacing in permanently paced patients with worsening heart failure: The RD-CHF Study. Pacing Clin Electrophysiol 2007;30 Suppl 1:S23-30. 
109. Delnoy PP, Ottervanger JP, Vos DH, Elvan A, Misier AR, Beukema WP, Steendijk P, van Hemel NM. Upgrading to biventricular pacing guided by pressure-volume loop analysis during implantation.J Cardiovasc Electrophysiol 2011;22:677-683.

110. Hoijer C], Meurling C, Brandt J. Upgrade to biventricular pacing in patients with conventional pacemakers and heart failure: a double-blind, randomized crossover study. Europace 2006;8:51-55.

111. van Geldorp IE, Vernooy K, Delhaas T, Prins MH, Crijns HJ, Prinzen FW, Dijkman B. Beneficial effects of biventricular pacing in chronically right ventricular paced patients with mild cardiomyopathy. Europace 2010;12:223-229.

112. Baker CM, Christopher TJ, Smith PF, Langberg JJ, Delurgio DB, Leon AR. Addition of a left ventricular lead to conventional pacing systems in patients with congestive heart failure: feasibility, safety, and early results in 60 consecutive patients. Pacing Clin Electrophysiol 2002;25:1166-1171.

113. Eldadah ZA, Rosen B, Hay I, Edvardsen T, Jayam V, Dickfeld T, Meininger GR, Judge DP, Hare J, Lima JB, Calkins H, Berger RD. The benefit of upgrading chronically right ventricle-paced heart failure patients to resynchronization therapy demonstrated by strain rate imaging. Heart Rhythm 2006;3:435-442.

114. Laurenzi F, Achilli A, Avella A, Peraldo C, Orazi S, Perego GB, Cesario A, ValsecchiS, De Santo T, Puglisi A, Tondo C. Biventricular upgrading in patients with conventional pacing system and congestive heart failure: results and response predictors. Pacing Clin Electrophysiol 2007;30:1096-1104.

115. Leon AR, Greenberg JM, Kanuru N, Baker CM, Mera FV, Smith AL, Langberg J], DeLurgio DB. Cardiac resynchronization in patients with congestive heart failure and chronic atrial fibrillation: effect of upgrading to biventricular pacing after chronic right ventricular pacing. J Am Coll Cardiol 2002;39:1258-1263.

116. Shimano M, Tsuji Y, Yoshida Y, Inden Y, Tsuboi N, Itoh T, Suzuki H, Muramatsu T, Okada T, Harata S, Yamada T, Hirayama H, Nattel S, Murohara T. Acute and chronic effects of cardiac resynchronization in patients developing heart failure with longterm pacemaker therapy for acquired complete atrioventricular block. Europace 2007;9:869-874.

117. Valls-Bertault V, Fatemi M, Gilard M, Pennec PY, Etienne Y, Blanc JJ. Assessment of upgrading to biventricular pacing in patients with right ventricular pacing and congestive heart failure after atrioventricular junctional ablation for chronic atrial fibrillation. Europace 2004;6:438-443.

118. Vatankulu MA, Goktekin O, Kaya MG, Ayhan S, Kucukdurmaz Z, Sutton R, Henein M. Effect of long-term resynchronization therapy on left ventricular remodelling in pacemaker patients upgraded to biventricular devices. Am J Cardiol 2009; 103:1280-1284

119. Foley PW, Muhyaldeen SA, Chalil S, Smith RE, Sanderson JE, Leyva F. Long-term effects of upgrading from right ventricular pacing to cardiac resynchronization therapy in patients with heart failure. Europace 2009;11:495-501.

120. Frohlich G, Steffel J, Hurlimann D, Enseleit F, Luscher TF, Ruschitzka F, Abraham WT, Holzmeister J. Upgrading to resynchronization therapy after chronic right ventricular pacing improves left ventricular remodelling. Eur Heart J 2010;31:1477-1485

121. Marai I, Gurevitz O, Carasso S, Nof E, Bar-Lev D, Luria D, Arbel Y, Freimark D, Feinberg MS, Eldar M, Glikson M. Improvement of congestive heart failure by upgrading of conventional to resynchronization pacemakers. Pacing Clin Electrophysiol 2006:29:880-884.

122. Paparella G, Sciarra L, Capulzini L, Francesconi A, De Asmundis C, Sarkozy A, Cazzin R, Brugada P. Long-term effects of upgrading to biventricular pacing: differences with cardiac resynchronization therapy as primary indication. Pacing Clin Electrophysiol 2010;33:841-849.

123. Albertsen AE, Nielsen JC, Poulsen SH, Mortensen PT, Pedersen AK, Hansen PS, Jensen HK, Egeblad H. Biventricular pacing preserves left ventricular performance in patients with high-grade atrio-ventricular block: a randomized comparison with DDD (R) pacing in 50 consecutive patients. Europace 2008;10:314-320.

124. Chan JY, Fang F, Zhang Q, Fung JW, Razali O, Azlan H, Lam KH, Chan HC, Yu CM. Biventricular pacing is superior to right ventricular pacing in bradycardia patients with preserved systolic function: 2-year results of the PACE trial. Eur Heart J 2011;32:2533-2540

125. Curtis AB, Worley S Adamson PB, Chung E, Niazi I, Sherfesee L, Shinn T, Sutton MS. Biventricular pacing for atrioventricular block and systolic dysfunction. New EnglJ Med 2013;368:1585-1593.

126. Curtis AB, Adamson PB, Chung E, Sutton MS, Tang F, Worley S. Biventricular versus right ventricular pacing in patients with AV block (BLOCK HF): clinical study design and rationale. J Cardiovasc Electrophysiol 2007;18:965-971.

127. Kindermann M, Hennen B, Jung J, Geisel J, Bohm M, Frohlig G. Biventricular versus conventional right ventricular stimulation for patients with standard pacing indication and left ventricular dysfunction: the Homburg Biventricular Pacing Evaluation (HOBIPACE). J Am Coll Cardiol 2006;47:1927-1937.

128. Martinelli Filho M, de Siqueira SF, Costa R, Greco OT, Moreira LF, D'Avila A, Heist EK. Conventional versus biventricular pacing in heart failure and bradyarrhythmia: the COMBAT study. J Card Fail 2010;16:293-300.
129. Stockburger M, Gomez-Doblas J), Lamas G, Alzueta J, Fernandez-Lozano I, Cobo E, Wiegand U, Concha JF, Navarro X, Navarro-Lopez F, de Teresa E. Preventing ventricular dysfunction in pacemaker patients without advanced heart failure: results from a multi-centre international randomized trial (PREVENT-HF). Eur 」 Heart Fail 2011;13:633-641.

130. Yu CM, Chan JY, Zhang Q, Omar R, Yip GW, Hussin A, Fang F, Lam KH, Chan HC, Fung JW. Biventricular pacing in patients with bradycardia and normal ejection fraction. N Engl J Med 2009;361:2123-2134.

131. Lam SK, Owen A. Combined resynchronisation and implantable defibrillator therapy in left ventricular dysfunction: Bayesian network meta-analysis of randomised controlled trials. BMJ 2007;335:925.

132. Gang UJ, Hvelplund A, Pedersen S, Iversen A, Jons C, Abildstrom SZ, Haarbo J, Jensen JS, Thomsen PE. High-degree atrioventricular block complicating ST-segment elevation myocardial infarction in the era of primary percutaneous coronary intervention. Europace 2012;14:1639-1645.

133. Behar S, Zissman E, Zion M, Hod H, Goldbourt U, Reicher-Reiss H, Shalev $Y$, Kaplinsky E, Caspi A. Prognostic significance of second-degree atrioventricular block in inferior wall acute myocardial infarction. SPRINT Study Group. Am J Cardiol 1993;72:831-834.

134. Col JJ, Weinberg SL. The incidence and mortality of intraventricular conduction defects in acute myocardial infarction. Am J Cardiol 1972;29:344-350.

135. Ginks WR, Sutton R, Oh W, Leatham A. Long-term prognosis after acute anterior infarction with atrioventricular block. Br Heart J 1977;39:186-189.

136. Jim MH, Chan AO, Tse HF, Barold SS, Lau CP. Clinical and angiographic findings of complete atrioventricular block in acute inferior myocardial infarction. Ann Acad Med Singapore 2010;39:185-190

137. Gross GJ, Chiu CC, Hamilton RM, Kirsh JA, Stephenson EA. Natural history of postoperative heart block in congenital heart disease: implications for pacing intervention. Heart Rhythm 2006;3:601-604

138. Krongrad E. Prognosis for patients with congenital heart disease and postoperative intraventricular conduction defects. Circulation 1978;57:867-870.

139. Murphy DA, Tynan M, Graham GR, Bonham-Carter RE. Prognosis of complete atrioventricular dissociation in children after open-heart surgery. Lancet 1970;1: $750-752$.

140. Squarcia U, Merideth J, McGoon DC, Weidman WH. Prognosis of transient atrioventricular conduction disturbances complicating open heart surgery for congenital heart defects. Am J Cardiol 1971;28:648-652.

141. Weindling SN, SaulJP, Gamble WJ, MayerJE, Wessel D, Walsh EP. Duration of complete atrioventricular block after congenital heart disease surgery. Am J Cardiol 1998;82:525-527.

142. Chang SM, Nagueh SF, Spencer WH 3rd, Lakkis NM. Complete heart block: determinants and clinical impact in patients with hypertrophic obstructive cardiomyopathy undergoing nonsurgical septal reduction therapy. I Am Coll Cardiol 2003;42 $296-300$.

143. Topilski I, Sherez J, Keren G, Copperman I. Long-term effects of dual-chamber pacing with periodic echocardiographic evaluation of optimal atrioventricular delay in patients with hypertrophic cardiomyopathy $>50$ years of age. Am J Cardiol 2006;97:1769-1775.

144. Kappenberger L, Linde C, Daubert C, McKenna W, Meisel E, Sadoul N, Chojnowska L, Guize L, Gras D, Jeanrenaud X, Ryden L. Pacing in hypertrophic obstructive cardiomyopathy. A randomized crossover study. PIC Study Group. Eur Heart J 1997;18:1249-1256.

145. Maron B], Nishimura RA, McKenna W], Rakowski H, Josephson ME, Kieval RS. Assessment of permanent dual-chamber pacing as a treatment for drug-refractory symptomatic patients with obstructive hypertrophic cardiomyopathy. A randomized, double-blind, crossover study (M-PATHY). Circulation 1999;99:2927-2933.

146. Nishimura RA, Trusty JM, Hayes DL, Ilstrup DM, Larson DR, Hayes SN, Allison TG, Tajik AJ. Dual-chamber pacing for hypertrophic cardiomyopathy: a randomized, double-blind, crossover trial. J Am Coll Cardiol 1997;29:435-441.

147. Galve E, Sambola A, Saldana G, Quispe I, Nieto E, Diaz A, Evangelista A, Candell-Riera J. Late benefits of dual-chamber pacing in obstructive hypertrophic cardiomyopathy: a 10-year follow-up study. Heart 2010;96:352-356.

148. Megevand A, Ingles J, Richmond DR, Semsarian C. Long-term follow-up of patients with obstructive hypertrophic cardiomyopathy treated with dual-chamber pacing. Am J Cardiol 2005;95:991-993.

149. Israel CW, Hugl B, Unterberg C, Lawo T, Kennis I, Hettrick D, Hohnloser SH. Pacetermination and pacing for prevention of atrial tachyarrhythmias: results from a multicenter study with an implantable device for atrial therapy.J Cardiovasc Electrophysiol 2001;12:1121-1128.

150. Blanc JJ, De Roy L, Mansourati J, Poezevara Y, Marcon JL, Schoels W, Hidden-Lucet F, Barnay C. Atrial pacing for prevention of atrial fibrillation: assessment of simultaneously implemented algorithms. Europace 2004;6:371-379.

151. Carlson MD, Ip J, Messenger J, Beau S, Kalbfleisch S, Gervais P, Cameron DA, Duran A, Val-Mejias J, Mackall J, Gold M. A new pacemaker algorithm for the 
treatment of atrial fibrillation: results of the Atrial Dynamic Overdrive Pacing Trial (ADOPT). J Am Coll Cardiol 2003;42:627-633.

152. Gold MR, Adler S, Fauchier L, Haffajee C, Ip J, Kainz W, Kawasaki R, Prakash A, Taborsky M, Waller T, Wilson V, Li S, Hoffmann E. Impact of atrial prevention pacing on atrial fibrillation burden: primary results of the Study of Atrial Fibrillation Reduction (SAFARI) trial. Heart Rhythm 2009;6:295-301.

153. Healey JS, Connolly SJ, Gold MR, Israel CW, Van Gelder IC, Capucci A, Lau CP, Fain E, Yang S, Bailleul C, Morillo CA, Carlson M, Themeles E, Kaufman ES, Hohnloser SH. Subclinical atrial fibrillation and the risk of stroke. N Engl J Med 2012;366:120-129.

154. Hohnloser SH, Healey JS, Gold MR, Israel CW, Yang S, van Gelder I, Capucci A, Lau CP, Fain E, Morillo CA, Ha A, Carlson M, Connolly SJ. Atrial overdrive pacing to prevent atrial fibrillation: insights from ASSERT. Heart Rhythm 2012;9:1667-1673.

155. Israel CW, Gronefeld G, Ehrlich JR, Li YG, Hohnloser SH. Prevention of immediate reinitiation of atrial tachyarrhythmias by high-rate overdrive pacing: results from a prospective randomized trial. J Cardiovasc Electrophysiol 2003;14:954-959.

156. Kantharia BK, Freedman RA, Hoekenga D, Tomassoni G, Worley S, Sorrentino R, Steinhaus D, Wolkowicz JM, Syed ZA. Increased base rate of atrial pacing for prevention of atrial fibrillation after implantation of a dual-chamber pacemaker: insights from the Atrial Overdrive Pacing Study. Europace 2007;9:1024-1030.

157. Lee MA, Weachter R, PollakS, Kremers MS, NaikAM, Silverman R, Tuzi J, Wang W, Johnson LJ, Euler DE. The effect of atrial pacing therapies on atrial tachyarrhythmia burden and frequency: results of a randomized trial in patients with bradycardia and atrial tachyarrhythmias. J Am Coll Cardiol 2003;41:1926-1932.

158. Mont L, Ruiz-Granell R, Martinez JG, Carmona JR, Fidalgo M, Cobo E, Riera M, Navarro X. Impact of anti-tachycardia pacing on atrial fibrillation burden when added on top of preventive pacing algorithms: results of the prevention or termination (POT) trial. Europace 2008;10:28-34.

159. Sulke N, Silberbauer J, Boodhoo L, Freemantle N, Kamalvand K, O’Nunain S, Hildick-Smith D, Lloyd GW, Patel NR, Paul V. The use of atrial overdrive and ventricular rate stabilization pacing algorithms for the prevention and treatment of paroxysmal atrial fibrillation: the Pacemaker Atrial Fibrillation Suppression (PAFS) study. Europace 2007;9:790-797.

160. Burke PT, Ghanbari H, Alexander PB, Shaw MK, Daccarett M, Machado C. A protocol for patients with cardiovascular implantable devices undergoing magnetic resonance imaging (MRI): should defibrillation threshold testing be performed post-(MRI). J Interv Card Electrophysiol 2010;28:59-66.

161. Del Ojo JL, Moya F, Villalba J, Sanz O, Pavon R, Garcia D, Pastor L. Is magnetic resonance imaging safe in cardiac pacemaker recipients? Pacing Clin Electrophysiol 2005; 28:274-278.

162. Gimbel JR, Kanal E, Schwartz KM, Wilkoff BL. Outcome of magnetic resonance imaging (MRI) in selected patients with implantable cardioverter defibrillators (ICDs). Pacing Clin Electrophysiol 2005;28:270-273.

163. Halshtok O, Goitein O, Abu Sham'a R, Granit H, Glikson M, Konen E. Pacemakers and magnetic resonance imaging: no longer an absolute contra-indication when scanned correctly. Isr Med Assoc J 2010;12:391-395.

164. Martin ET, Coman JA, Shellock FG, Pulling CC, Fair R, Jenkins K. Magnetic resonance imaging and cardiac pacemaker safety at 1.5-Tesla.J Am Coll Cardiol 2004;43:1315-1324.
165. Mollerus M, Albin G, Lipinski M, Lucca J. Cardiac biomarkers in patients with permanent pacemakers and implantable cardioverter-defibrillators undergoing an MRI scan. Pacing Clin Electrophysiol 2008;31:1241-1245.

166. Mollerus M, Albin G, Lipinski M, Lucca J. Magnetic resonance imaging of pacemakers and implantable cardioverter-defibrillators without specific absorption rate restrictions. Europace 2010;12:947-951.

167. Naehle CP, Meyer C, Thomas D, Remerie S, Krautmacher C, Litt H, Luechinger R, Fimmers R, Schild H, Sommer T. Safety of brain 3-T MR imaging with transmit-receive head coil in patients with cardiac pacemakers: pilot prospective study with 51 examinations. Radiology 2008;249:991-1001.

168. Naehle CP, Strach K, Thomas D, Meyer C, Linhart M, Bitaraf S, Litt H, Schwab JO, Schild $\mathrm{H}$, Sommer T. Magnetic resonance imaging at 1.5-T in patients with implantable cardioverter-defibrillators. J Am Coll Cardiol 2009;54:549-555.

169. Naehle CP, Zeijlemaker V, Thomas D, Meyer C, Strach K, Fimmers R, Schild H, Sommer T. Evaluation of cumulative effects of $M R$ imaging on pacemaker systems at 1.5 Tesla. Pacing Clin Electrophysiol 2009;32:1526-1535.

170. Nazarian S, Hansford R, Roguin A, Goldsher D, Zviman MM, Lardo AC, Caffo BS, Frick KD, Kraut MA, Kamel IR, Calkins H, Berger RD, Bluemke DA, Halperin HR. A prospective evaluation of a protocol for magnetic resonance imaging of patients with implanted cardiac devices. Ann Intern Med 2011;155:415-424.

171. Sommer T, Naehle CP, Yang A, Zeijlemaker V, Hackenbroch M, Schmiedel A Meyer C, Strach K, Skowasch D, Vahlhaus C, Litt H, Schild H. Strategy for safe performance of extrathoracic magnetic resonance imaging at 1.5 tesla in the presence of cardiac pacemakers in non-pacemaker-dependent patients: a prospective study with 115 examinations. Circulation 2006;114:1285-1292.

172. Strach K, Naehle CP, Muhlsteffen A, Hinz M, Bernstein A, Thomas D, Linhart M, Meyer C, Bitaraf S, Schild H, Sommer T. Low-field magnetic resonance imaging: increased safety for pacemaker patients? Europace 2010;12:952-960.

173. Wilkoff BL, Bello D, Taborsky M, Vymazal J, Kanal E, Heuer H, Hecking K, Johnson WB, Young W, Ramza B, Akhtar N, Kuepper B, Hunold P, Luechinger R, Puererfellner H, Duru F, Gotte MJ, Sutton R, Sommer T. Magnetic resonance imaging in patients with a pacemaker system designed for the magnetic resonance environment. Heart Rhythm 2011;8:65-73.

174. Crossley GH, Boyle A, Vitense H, Chang Y, Mead RH. The CONNECT (Clinical Evaluation of Remote Notification to Reduce Time to Clinical Decision) trial: the value of wireless remote monitoring with automatic clinician alerts. J Am Coll Cardiol 2011:57:1181-1189.

175. Landolina M, Perego GB, Lunati M, Curnis A, Guenzati G, Vicentini A, Parati G, Borghi G, Zanaboni P, Valsecchi S, Marzegalli M. Remote monitoring reduces healthcare use and improves quality of care in heart failure patients with implantable defibrillators: the evolution of management strategies of heart failure patients with implantable defibrillators (EVOLVO) study. Circulation 2012;125: 2985-2992.

176. Varma N, Epstein AE, Irimpen A, Schweikert R, Love C. Efficacy and safety of automatic remote monitoring for implantable cardioverter-defibrillator follow-up: the Lumos-T Safely Reduces Routine Office Device Follow-up (TRUST) trial. Circulation 2010;122:325-332. 